\title{
Measurement of the weak mixing angle with the Drell-Yan process in proton-proton collisions at the $\mathrm{LHC}$
}

\author{
S. Chatrchyan et al.* \\ (CMS Collaboration)
}

(Received 14 October 2011; published 8 December 2011)

\begin{abstract}
A multivariate likelihood method to measure electroweak couplings with the Drell-Yan process at the LHC is presented. The process is described by the dilepton rapidity, invariant mass, and decay angle distributions. The decay angle ambiguity due to the unknown assignment of the scattered constituent quark and antiquark to the two protons in a collision is resolved statistically using correlations between the observables. The method is applied to a sample of dimuon events from proton-proton collisions at $\sqrt{s}=7 \mathrm{TeV}$ collected by the CMS experiment at the LHC, corresponding to an integrated luminosity of $1.1 \mathrm{fb}^{-1}$. From the dominant $u \bar{u}, d \bar{d} \rightarrow \gamma^{*} / Z \rightarrow \mu^{-} \mu^{+}$process, the effective weak mixing angle parameter is measured to be $\sin ^{2} \theta_{\text {eff }}=0.2287 \pm 0.0020$ (stat.) \pm 0.0025 (syst.). This result is consistent with measurements from other processes, as expected within the standard model.
\end{abstract}

DOI: 10.1103/PhysRevD.84.112002

PACS numbers: 12.15.-y, 13.88.+e, 14.70.-e

\section{INTRODUCTION}

The main goal of the Large Hadron Collider (LHC) [1] is to explore physics at the $\mathrm{TeV}$ energy scale via protonproton collisions. The Drell-Yan process [2] occurs through the annihilation of a quark from one proton with an antiquark from the other proton, creating a virtual neutral gauge boson $\left(\gamma^{*}\right.$ or $Z$ ) that subsequently decays to a pair of oppositely charged leptons, as shown in Fig. 1. This annihilation process could reveal the existence of a new neutral gauge boson [3,4] or uncover deviations from the standard model of particle physics (SM) in elementary fermion couplings to the known neutral electroweak bosons. Besides quark-antiquark annihilation, new resonances decaying into lepton pairs may also be produced at the LHC via the gluon-fusion mechanism.

Analysis of electron-positron annihilations into dilepton or $b \bar{b}$ pairs at LEP and SLC [5] led to high-precision measurements of the electroweak Z-boson couplings to fermions. The measurement of the weak mixing angle parameter $\sin ^{2} \theta_{W}$ was performed to a precision of $\sim 0.1 \%$. In the SM, the weak mixing angle $\theta_{W}$ describes the rotation of the original $W^{0}$ and $B^{0}$ vector boson states into the observed $\gamma$ or $Z$ bosons as a result of spontaneous symmetry breaking [6]. Within the $\mathrm{SM}, \sin ^{2} \theta_{W}$ is the only free parameter that fixes the relative couplings of all fermions to the $\gamma$ or $Z$.

Measurements of the weak mixing angle with different initial and final fermion-antifermion states $f_{1} \bar{f}_{1} \rightarrow$ $\gamma^{*} / Z \rightarrow f_{2} \bar{f}_{2}$ tests the universality of the fermion/

*Full author list given at the end of the article.

Published by the American Physical Society under the terms of the Creative Commons Attribution 3.0 License. Further distribution of this work must maintain attribution to the author(s) and the published article's title, journal citation, and DOI. gauge-boson interactions and predictions of the SM. For example, LEP measurements [5] of the inclusive hadronic charge asymmetry provided a measurement of the couplings of light quarks compared to leptons and $b$ quarks. The NuTeV collaboration measured $\sin ^{2} \theta_{W}$ to precision of about $1 \%$ from neutrino and antineutrino deep inelastic scattering on nucleons [7]. The CDF and D0 experiments [8-10] at the Tevatron reached a similar precision with the Drell-Yan process in protonantiproton collisions, as did the $\mathrm{H} 1$ experiment [11] with electron-proton scattering at HERA. In this paper, we measure the $\sin ^{2} \theta_{W}$ parameter to a precision $\sim 1 \%$ in the proton-proton Drell-Yan process at the LHC with the CMS experiment.

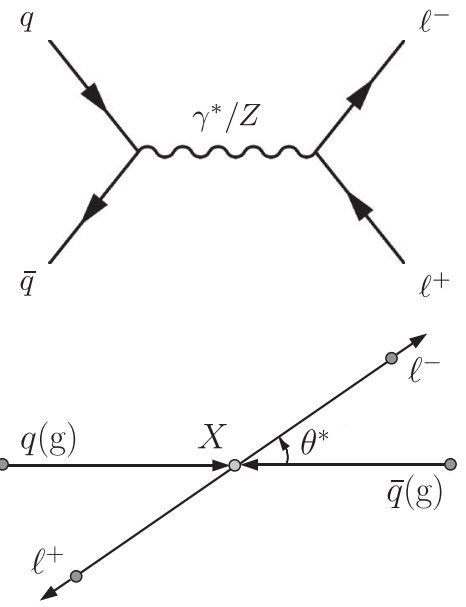

FIG. 1. Top: diagram describing the SM process $q \bar{q} \rightarrow$ $\gamma^{*} / Z \rightarrow \ell^{-} \ell^{+}$. Bottom: definition of the angle $\theta^{*}$ in the production and decay of an intermediate state $X$, such as $g g$ or $q \bar{q} \rightarrow X \rightarrow \ell^{-} \ell^{+}$. 
The proton-proton collisions at the LHC pose new challenges compared to previous collider experiments. The interference of the axial-vector and vector couplings leads to an asymmetry in the distribution of the polar angle of the lepton with respect to the direction of the constituent quark from the incoming proton in the quark-antiquark annihilation. This type of "forward-backward" asymmetry has been the primary measurement used to extract the couplings and $\sin ^{2} \theta_{W}$ at the LEP, SLC, and Tevatron experiments. However, because of the symmetric proton-proton collision at the LHC, the direction of the quark is not known and can be deduced only on a statistical basis [4,12-14] using the boost direction of the dilepton final state, because of the higher probability for a valence quark from one of the incoming protons to provide the boost. We have developed methods that allow a per-event analytical likelihood description to extract the maximal information about the process at the LHC. This technique exploits more information than the conventional forward-backward asymmetry approach, and the distribution of the polar angle as a function of both dilepton rapidity and mass is an essential component of this method. The technique has applications both for high-precision electroweak measurements and for rare-process searches at the LHC.

In this paper, we present a multivariate analysis that uses the full information about the Drell-Yan process $q \bar{q} \rightarrow$ $\gamma^{*} / Z \rightarrow \ell^{-} \ell^{+}$, parameterized as a function of the dilepton rapidity $Y$, invariant mass squared $\hat{s}$, and decay angle $\theta^{*}$. This process offers a relatively simple environment for the development of the matrix-element analysis techniques for resonance polarization studies at the LHC. Encouraged by feasibility studies of an analytical matrix-element approach in Ref. [15], we use a formalism based on an analytical description of the elementary interaction (Sec. II). The method is applied to a sample of protonproton collisions at a center-of-mass energy $\sqrt{s}=7 \mathrm{TeV}$, corresponding to an integrated luminosity of $1.1 \mathrm{fb}^{-1}$ recorded by the CMS experiment (Sec. III). We include a description of detector effects in the analytical likelihood model (Sec. IV) and pay particular attention to systematic effects (Sec. V). The result is consistent with measurements in other processes, as expected within the SM (Sec. VI).

\section{PHENOMENOLOGY OF THE DRELL-YAN PROCESS AT THE LHC}

The philosophy of the multivariate likelihood analysis is to first produce a phenomenological model of the process and then introduce detector effects into the model. The parameters of the model may either be fixed to the best known values or left free in the fit, to be determined from data. These parameters may include the physical quantities of interest, such as $\sin ^{2} \theta_{W}$, or a description of detector effects, such as a correction for the momentum scale in the track reconstruction. Therefore, we start with a discussion of a phenomenological model, and then proceed to detector-specific effects in the application of the analysis to CMS data.

The tree-level coupling of a spin-one gauge-boson to fermions is described by

$$
\epsilon^{\mu} \bar{u}_{f} \gamma_{\mu} e\left(\rho_{V}-\rho_{A} \gamma_{5}\right) v_{f}
$$

where $v_{f}$ and $\bar{u}_{f}$ are the Dirac spinors of the fermion $(f)$ and antifermion $(\bar{f}), \epsilon^{\mu}$ is the polarization vector of the spin-one boson, and $\rho_{V}$ and $\rho_{A}$ are the vector and axialvector couplings. The couplings of the SM gauge bosons $\gamma$ and $Z$ are given in Table I. In the limit of negligible fermion masses, which is a good approximation for both quarks and leptons in the Drell-Yan process near the Z-boson mass, only two helicity states of the fermions are possible. They correspond to amplitudes $A_{\uparrow \downarrow} \propto\left(\rho_{V}-\rho_{A}\right)$ and $A_{\downarrow \uparrow} \propto$ $-\left(\rho_{V}+\rho_{A}\right)$.

The parton-level cross section for the Drell-Yan process can be expressed with the help of the Wigner $d_{m, m^{\prime}}^{J}$ matrix, assuming the spin $J=1$ intermediate states $\gamma^{*}, Z$, and possible new unknown contributions (indicated by an ellipsis below), as

$$
\begin{aligned}
& \hat{\sigma}_{q \bar{q}}\left(\hat{s}, \cos \theta^{*} ; \theta_{W}\right) \\
& \propto \frac{1}{\hat{s}} \sum_{\chi_{1}, \chi_{2}, \lambda_{1}, \lambda_{2}=\uparrow, \downarrow}(2 J+1)\left(d_{\chi_{1}-\chi_{2}, \lambda_{1}-\lambda_{2}}^{J=1}\left(\theta^{*}\right)\right)^{2} \\
& \quad \times \mid A_{\chi_{1}, \chi_{2}}^{q \bar{q} \rightarrow \gamma} A_{\lambda_{1}, \lambda_{2}}^{\gamma \rightarrow \ell \ell}+A_{\chi_{1}, \chi_{2}}^{q \bar{q} \rightarrow Z}\left(\theta_{W}\right) A_{\lambda_{1}, \lambda_{2}}^{Z \rightarrow \ell \ell}\left(\theta_{W}\right) \\
& \quad \times \frac{\hat{s}}{\left(\hat{s}-m^{2}\right)+i m_{Z} \Gamma_{Z}}+\left.\ldots\right|^{2},
\end{aligned}
$$

where $\chi_{1}, \chi_{2}, \lambda_{1}$, and $\lambda_{2}$ are the helicity states of the quark, antiquark, lepton, and antilepton, and the dilepton decay angle $\theta^{*}$ is defined in the center-of-mass frame of the dilepton system as shown in Fig. 1. The effects of transverse motion of the incoming constituent quark and antiquark in their annihilation are minimized by using the Collins-Soper frame [17]. In this frame, the angle $\theta^{*}$ is defined as the angle between the lepton momentum and a $z^{\prime}$-axis that bisects the angle between the direction

TABLE I. Vector and axial-vector couplings of the SM gauge bosons to the charged fermion fields [16].

\begin{tabular}{lcc}
\hline \hline & $\rho_{V}$ & $\rho_{A}$ \\
\hline$\gamma \rightarrow e^{-} e^{+}, \mu^{-} \mu^{+}, \tau^{-} \tau^{+}$ & -1 & 0 \\
$\gamma \rightarrow u \bar{u}, c \bar{c}, t \bar{t}$ & $+2 / 3$ & 0 \\
$\gamma \rightarrow d \bar{d}, s \bar{s}, b \bar{b}$ & $-1 / 3$ & 0 \\
$Z \rightarrow e^{-} e^{+}, \mu^{-} \mu^{+}, \tau^{-} \tau^{+}$ & $\frac{-3+12 \sin ^{2} \theta_{W}}{6 \sin ^{2}\left(2 \theta_{W}\right)}$ & $\frac{-1}{2 \sin \left(2 \theta_{W}\right)}$ \\
$Z \rightarrow u \bar{u}, c \bar{c}, t \bar{t}$ & $\frac{+3-8 \sin ^{2} \theta_{W}}{6 \sin ^{2}\left(2 \theta_{W}\right)}$ & $\frac{+1}{2 \sin \left(2 \theta_{W}\right)}$ \\
$Z \rightarrow d \bar{d}, s \bar{s}, b \bar{b}$ & $\frac{-3+4 \sin ^{2} \theta_{W}}{6 \sin \left(2 \theta_{W}\right)}$ & $\frac{-1}{2 \sin \left(2 \theta_{W}\right)}$ \\
\hline \hline
\end{tabular}


of one proton and the direction opposite that of the other proton in the dilepton rest frame. Should there be new states contributing to the cross section with the same or different spin, they will either contribute with interference if produced in $q \bar{q}$ annihilation, or without interference if produced in gluon fusion $(g g)$, for example.

The parton-level cross section in Eq. (2) can be further simplified for the SM intermediate states $\gamma^{*}$ and $Z$ with $\operatorname{spin} J=1$ as

$$
\begin{aligned}
\hat{\sigma}_{q \bar{q}}\left(\hat{s}, \cos \theta^{*} ; \theta_{W}\right) \propto & \frac{3\left(\rho_{V}^{q \bar{q} \rightarrow \gamma}\right)^{2}\left(\rho_{V}^{\gamma \rightarrow \ell \ell}\right)^{2}}{2 \hat{s}} \times\left(1+\cos ^{2} \theta^{*}\right)+\frac{3}{2} \frac{\hat{s}}{\left(\hat{s}-m_{Z}^{2}\right)^{2}+m_{Z}^{2} \Gamma_{Z}^{2}} \\
& \times\left[\left(\left(\rho_{V}^{q \bar{q} \rightarrow Z}\right)^{2}+\left(\rho_{A}^{q \bar{q} \rightarrow Z}\right)^{2}\right)\left(\left(\rho_{V}^{Z \rightarrow \ell \ell}\right)^{2}+\left(\rho_{A}^{Z \rightarrow \ell \ell}\right)^{2}\right)\left(1+\cos ^{2} \theta^{*}\right)+8 \rho_{V}^{q \bar{q} \rightarrow Z} \rho_{A}^{q \bar{q} \rightarrow Z} \rho_{V}^{Z \rightarrow \ell \ell} \rho_{A}^{Z \rightarrow \ell \ell} \cos \theta^{*}\right] \\
& +\frac{3\left(\hat{s}-m_{Z}^{2}\right) \rho_{V}^{q \bar{q} \rightarrow \gamma} \rho_{V}^{\gamma \rightarrow \ell \ell}}{\left(\hat{s}-m_{Z}^{2}\right)^{2}+m_{Z}^{2} \Gamma_{Z}^{2}} \times\left[\rho_{V}^{q \bar{q} \rightarrow Z} \rho_{V}^{Z \rightarrow \ell \ell}\left(1+\cos ^{2} \theta^{*}\right)+2 \rho_{A}^{q \bar{q} \rightarrow Z} \rho_{A}^{Z \rightarrow \ell \ell} \cos \theta^{*}\right] .
\end{aligned}
$$

Equation (3) implies that the asymmetry in the polar angle arises from terms linear in $\cos \theta^{*}$. The value of the asymmetry depends on the dilepton invariant mass $m=$ $\sqrt{\hat{s}}$, quark flavor $q$, and weak mixing angle $\theta_{W}$. It could also be affected by deviations of couplings from SM expectations or by the presence of new contributions.

The differential cross section of the proton-proton Drell-Yan process can be expressed as a product of the parton-level cross section in Eqs. (2) or (3) and the parton distribution functions (PDFs) $f_{a}\left(x_{i}, \hat{s}\right)[18]$ describing the probability for partons of type $a$ to have a fraction $x_{i}$ of the proton momentum $p=\sqrt{s} / 2$ :

$$
\begin{aligned}
& \frac{d \sigma_{p p}\left(p x_{1}, p x_{2}, \cos \theta^{*} ; \theta_{W}\right)}{d x_{1} d x_{2} d \cos \theta^{*}} \\
& \propto \sum_{q=u, d, s, c, b}\left(\hat{\sigma}_{q \bar{q}}\left(\hat{s}, \operatorname{sgn}\left(x_{1}-x_{2}\right) \cos \theta^{*} ; \theta_{W}\right) f_{q}\left(x_{1}, \hat{s}\right) f_{\bar{q}}\left(x_{2}, \hat{s}\right)\right. \\
& \left.\quad+\hat{\sigma}_{q \bar{q}}\left(\hat{s}, \operatorname{sgn}\left(x_{2}-x_{1}\right) \cos \theta^{*} ; \theta_{W}\right) f_{q}\left(x_{2}, \hat{s}\right) f_{\bar{q}}\left(x_{1}, \hat{s}\right)\right) .
\end{aligned}
$$

The expression $\operatorname{sgn}\left(x_{1}-x_{2}\right)$ refers to the sign of the difference $\left(x_{1}-x_{2}\right)$, reflecting the fact that the quark direction is assumed to coincide with the boost of the $q \bar{q}$ system. This assumption introduces a dilution in the odd-power terms in $\cos \theta^{*}$.

It is convenient to convert from the two variables $\left(x_{1}, x_{2}\right)$ to $(Y, \hat{s})$, the dilepton rapidity and the square of the dilepton mass, as

$$
\begin{gathered}
Y=\frac{1}{2} \ln \left(\frac{\hat{E}+\hat{p}_{z}}{\hat{E}-\hat{p}_{z}}\right)=\frac{1}{2} \ln \left(\frac{x_{1}}{x_{2}}\right), \\
\hat{s}=\hat{E}^{2}-\hat{p}^{2}=x_{1} x_{2} s,
\end{gathered}
$$

where $\hat{E}$ is the dilepton system energy, $\hat{p}$ and $\hat{p}_{z}$ are its momentum and longitudinal momentum in the laboratory frame, and $\hat{p}=\left|\hat{p}_{z}\right|$ at leading order in QCD.

After transformation of the variables, the Drell-Yan process in proton-proton interactions can be expressed as follows:

$$
\begin{aligned}
& \frac{d \sigma_{p p}\left(Y, \hat{s}, \cos \theta^{*} ; \theta_{W}\right)}{d Y d \hat{s} d \cos \theta^{*}} \\
& \propto \sum_{q=u, d, s, c, b}\left[\hat{\sigma}_{q \bar{q}}^{\text {even }}\left(\hat{s}, \cos ^{2} \theta^{*} ; \theta_{W}\right)\right. \\
& \left.\quad+D_{q \bar{q}}(\hat{s}, Y) \times \hat{\sigma}_{q \bar{q}}^{\text {odd }}\left(\hat{s}, \cos \theta^{*} ; \theta_{W}\right)\right] \times F_{q \bar{q}}(\hat{s}, Y) .
\end{aligned}
$$

The parton factor is defined as

$$
\begin{aligned}
F_{q \bar{q}}(\hat{s}, Y)= & f_{q}\left(e^{+Y} \sqrt{\hat{s} / s}, \hat{s}\right) f_{\bar{q}}\left(e^{-Y} \sqrt{\hat{s} / s}, \hat{s}\right) \\
& +f_{q}\left(e^{-Y} \sqrt{\hat{s} / s}, \hat{s}\right) f_{\bar{q}}\left(e^{+Y} \sqrt{\hat{s} / s}, \hat{s}\right),
\end{aligned}
$$

and the dilution factor is defined as

$$
D_{q \bar{q}}(\hat{s}, Y)=\frac{f_{q}\left(e^{+|Y|} \sqrt{\hat{s} / s}, \hat{s}\right) f_{\bar{q}}\left(e^{-|Y|} \sqrt{\hat{s} / s}, \hat{s}\right)-f_{q}\left(e^{-|Y|} \sqrt{\hat{s} / s}, \hat{s}\right) f_{\bar{q}}\left(e^{+|Y|} \sqrt{\hat{s} / s}, \hat{s}\right)}{f_{q}\left(e^{+Y} \sqrt{\hat{s} / s}, \hat{s}\right) f_{\bar{q}}\left(e^{-Y} \sqrt{\hat{s} / s}, \hat{s}\right)+f_{q}\left(e^{-Y} \sqrt{\hat{s} / s}, \hat{s}\right) f_{\bar{q}}\left(e^{+Y} \sqrt{\hat{s} / s}, \hat{s}\right)}
$$

The two components of the parton cross section contain either an even or odd power of $\cos \theta^{*}$

$$
\begin{aligned}
\hat{\sigma}_{q \bar{q}}^{\mathrm{even}}\left(\hat{s}, \cos ^{2} \theta^{*} ; \theta_{W}\right)= & \frac{1}{2}\left(\hat{\sigma}_{q \bar{q}}\left(\hat{s},+\cos \theta^{*} ; \theta_{W}\right)\right. \\
& \left.+\hat{\sigma}_{q \bar{q}}\left(\hat{s},-\cos \theta^{*} ; \theta_{W}\right)\right),
\end{aligned}
$$

$$
\begin{aligned}
\hat{\sigma}_{q \bar{q}}^{\text {odd }}\left(\hat{s}, \cos \theta^{*} ; \theta_{W}\right)= & \frac{1}{2}\left(\hat{\sigma}_{q \bar{q}}\left(\hat{s},+\cos \theta^{*} ; \theta_{W}\right)\right. \\
& \left.-\hat{\sigma}_{q \bar{q}}\left(\hat{s},-\cos \theta^{*} ; \theta_{W}\right)\right) .
\end{aligned}
$$

The factors $F_{q \bar{q}}(\hat{s}, Y)$ and $D_{q \bar{q}}(\hat{s}, Y)$ both arise from the PDFs and can be extracted from parameterizations such as in Refs. [19-22]. We choose to extract PDFs numerically from the leading-order (LO) parameterization CTEQ6 [19] 
to match the LO model of the process. We parameterize the PDFs analytically using polynomial and exponential functions in the relevant range of $x$ and with coefficients that are also functions of $\hat{s}$. The relevant range of $x$ for this analysis is $1.1 \times 10^{-3}<x<1.4 \times 10^{-1}$, motivated by the detector acceptance and the dilepton mass selection criteria presented in Sec. IV. This analytical parameterization is illustrated in Fig. 2. We choose an analytical parameterization because of the computational speed and ease of implementation. Its performance has been cross-checked with numerical computations. For systematic uncertainty studies, we check the performance against simulations with other PDF models [18,23], such as next-to-leading-order (NLO) CTEQ [20], MSTW [21], and NNPDF [22].

The function $F_{q \bar{q}}(\hat{s}, Y)$ is the effective cross section factor that scales the elementary parton-level cross section. This factor quickly decreases as the energy scale approaches values comparable to the full proton energies, as illustrated in Fig. 3 for $Y=0$ production. The factor $D_{q \bar{q}}(\hat{s}, Y)$ reflects the fact that the quark direction is generally unknown and is taken as the boost direction of the dilepton system, because of the higher probability for valence quarks to provide the boost. For $q=u$ or $d$ this factor ranges between 0 and 1 as $|Y|$ changes from 0 to 4 , as illustrated in Fig. 4 for $\hat{s}$ around the $Z$ pole. From Eq. (9) it follows that $D_{q \bar{q}}=0$ for $q=s, c, b$ under the assumption $f_{\bar{q}}\left(x, Q^{2}\right)=f_{q}\left(x, Q^{2}\right)$, which is a good approximation in the current PDF model. A challenge at the LHC is that the dilution factor $D_{q \bar{q}}$ is small for the typical range of $Y$ values in the detector acceptance region, as discussed below.

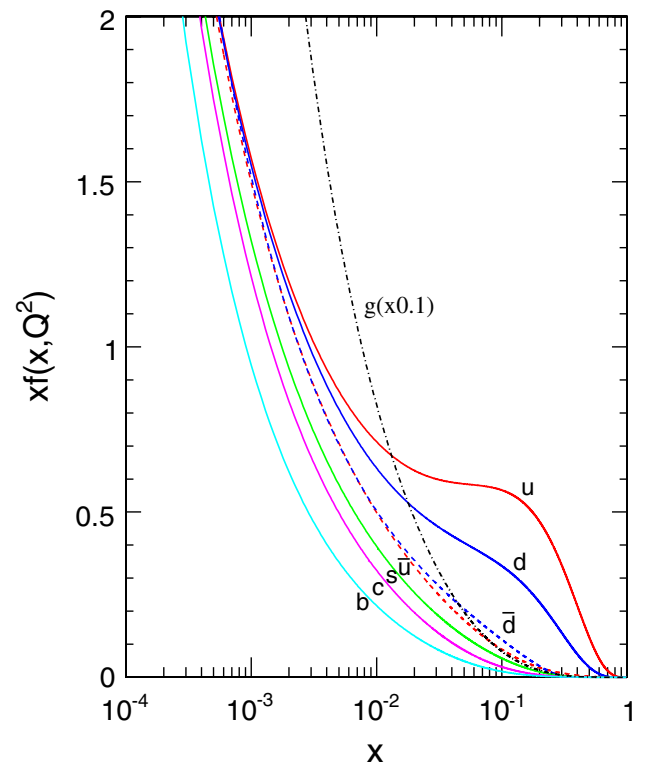

FIG. 2 (color online). Analytical parameterization of the parton distribution functions $x f_{a}\left(x, Q^{2}\right)$ at $Q=100 \mathrm{GeV}$ using the CTEQ6 [19] numerical computation for the various quarks, antiquarks, and the gluon. The gluon distribution is scaled by a factor of 0.1 .

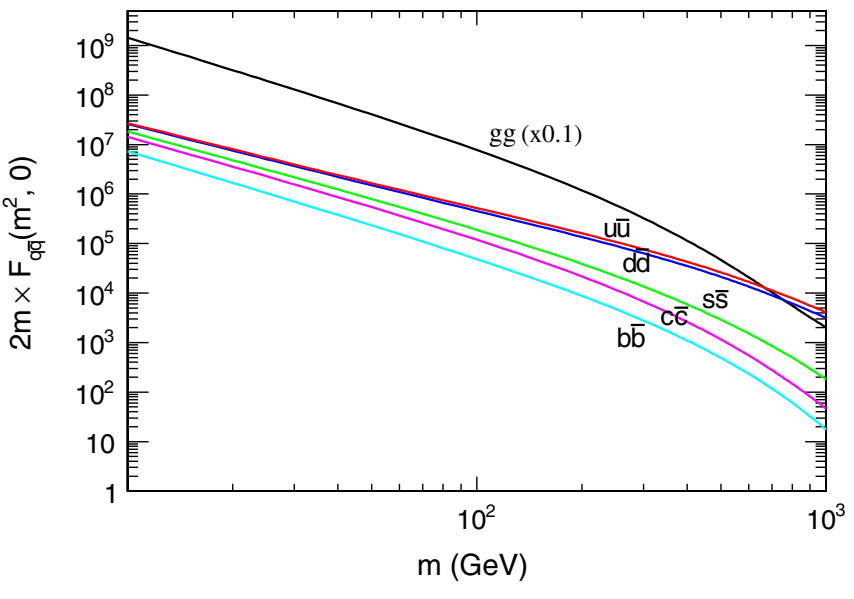

FIG. 3 (color online). Distribution of the effective cross section factor $2 m F_{q \bar{q}}\left(m^{2}, Y=0\right)$ defined in Eq. (8) for five quark flavors (from top to bottom $q=u, d, s, c, b$ ) for proton-proton collision energies of $7 \mathrm{TeV}$ as a function of the dilepton mass $m$. An equivalent factor for gluon-fusion production is shown for comparison and is scaled by a factor of 0.1 .

Information about $\sin ^{2} \theta_{W}$ or individual fermion couplings is contained in the shape of the three-dimensional distributions in Eq. (7) and enters through the elementary couplings of the electroweak bosons and fermions in the process $q \bar{q} \rightarrow \gamma^{*} / Z \rightarrow \ell^{-} \ell^{+}$.

Figure 5 illustrates the projections of the differential cross section from Eq. (7) in $Y, m=\sqrt{\hat{s}}$, and $\cos \theta^{*}$ for the five different quark flavors and combined. The relative fractions of the different quark flavors are 0.450, 0.375, $0.103,0.048$, and 0.025 for $u \bar{u}, d \bar{d}, s \bar{s}, c \bar{c}$, and $b \bar{b}$, respectively. The results of the analytical model leading to Eq. (7) show good agreement with the predictions from conventional LO numerical Monte Carlo (MC) simulation using

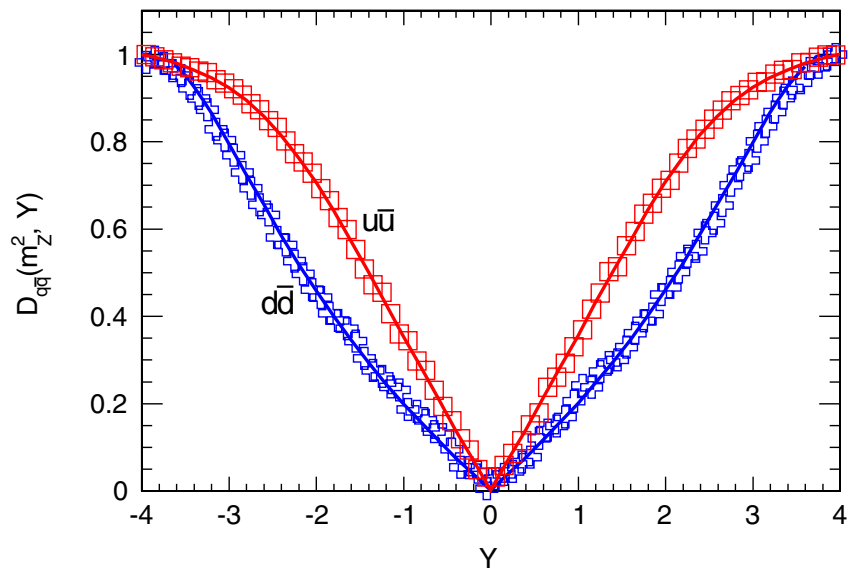

FIG. 4 (color online). The dilution factor $D_{q \bar{q}}\left(\hat{s}=m_{Z}^{2}, Y\right)$ for $u \bar{u}$ (top, red boxes) and $d \bar{d}$ (bottom, blue crosses) production as a function of the dilepton rapidity $Y$. The prediction from the PYTHIA simulation (boxes and crosses) of the $q \bar{q} \rightarrow \gamma^{*} / Z \rightarrow$ $\mu^{-} \mu^{+}$process and the analytical distributions (solid curves) from Eq. (9) are shown. 

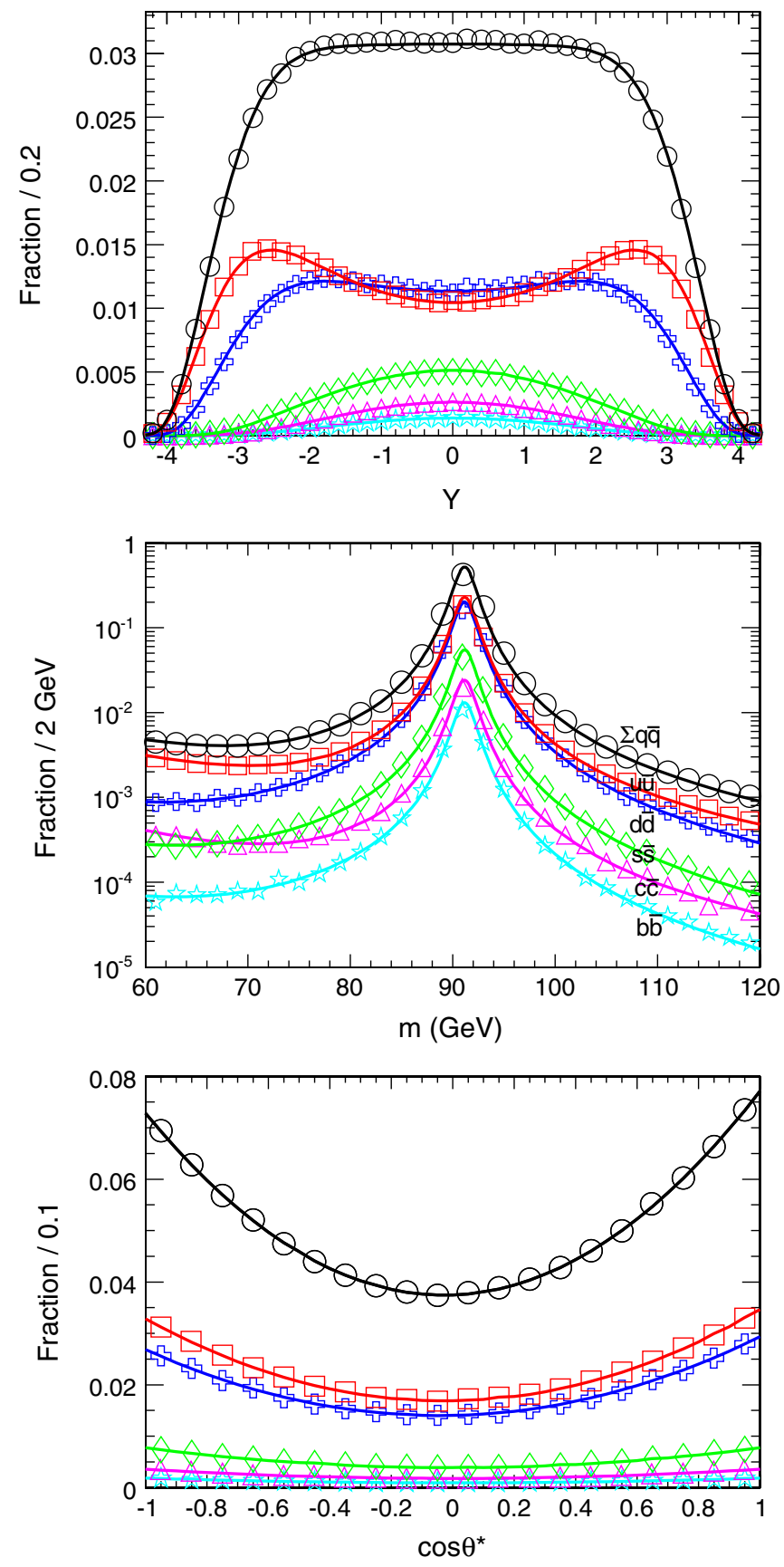

FIG. 5 (color online). Distributions of $Y$ (top), $m$ (middle), and $\cos \theta^{*}$ (bottom), from PYTHIA simulation (points) of the $q \bar{q} \rightarrow$ $\gamma^{*} / Z \rightarrow \mu^{-} \mu^{+}$process and analytical distributions from Eq. (7). Distributions for five quark flavors are shown combined $\Sigma q \bar{q}$ (black circles) and separately, in order of decreasing contribution: $u \bar{u}$ (red boxes), $d \bar{d}$ (blue crosses), $s \bar{s}$ (green diamonds), $c \bar{c}$ (magenta triangles), and $b \bar{b}$ (cyan stars). Distributions are normalized to unit area and are shown as fractions of events per bin.

the PYTHIA generator [24] with LO CTEQ6 [19] PDFs. The above cross section is parameterized at leading order in both strong $(\mathrm{QCD})$ and electroweak (EWK) interactions. Effects from NLO QCD contributions are studied with a detailed NLO POWHEG [25-27] simulation, which includes contributions from both initial-state gluon radiation and quark-gluon scattering. Effects from NLO EWK contributions are expected to be small compared to the precision of this analysis. EWK corrections are absorbed in a definition of the effective weak mixing parameter, $\sin ^{2} \theta_{\text {eff }}[16]$. We use $\sin ^{2} \theta_{\text {eff }}$ in place of $\sin ^{2} \theta_{W}$ for the rest of this paper.

We apply the above technique to the measurement of the weak mixing angle. We take the SM description of electroweak interactions and PDFs in the proton as given, and allow only the effective weak mixing angle $\theta_{\text {eff }}$ to be unconstrained. More generally, the above formalism with the multivariate analysis of the Drell-Yan process allows us to study the elementary couplings of fermions to electroweak neutral fields, such as $\gamma^{*} / Z$ in the SM, as well as the structure functions of the proton.

\section{DETECTION OF THE DRELL-YAN EVENTS WITH CMS}

We apply the above method in an analysis of the $q \bar{q} \rightarrow$ $\gamma^{*} / Z \rightarrow \mu^{-} \mu^{+}$process and measure $\sin ^{2} \theta_{\text {eff }}$. The choice of $\mu^{-} \mu^{+}$, as opposed to $e^{-} e^{+}$, is motivated by the more reliable description of the detector and background effects, as well as the fact that this final state has not yet been studied for $\sin ^{2} \theta_{\text {eff }}$ measurements in $q \bar{q}$ interactions. However, we do not expect any limitations in the method for future application to other final states. The expected multivariate distributions in Eq. (7) are modified by smearing due to detector resolution and photon final-state radiation (FSR), and by acceptance effects and nonuniform reconstruction as a function of the observables. All these effects are taken into account in the analytical parameterization, as shown below.

A detailed description of the CMS detector can be found in Ref. [28]. The central feature of CMS is a $3.8 \mathrm{~T}$ superconducting solenoid of $6 \mathrm{~m}$ internal diameter. Within the field volume are the silicon pixel and strip tracker, the crystal electromagnetic calorimeter (ECAL), and the brass/scintillator hadron calorimeter (HCAL). This analysis of the dimuon final state does not rely strongly on ECAL or HCAL measurements. Muons are measured in the window $|\eta|<2.5$ with the tracker and muon system. The pseudorapidity $\eta$ is defined as $\ln \cot (\theta / 2)$ with the polar angle $\theta$ measured in the laboratory frame.

The silicon tracking detector (tracker) [29] consists of 1440 silicon pixel and 15148 silicon strip detector modules. The pixel modules provide two-dimensional measurements of the hit position in the module planes, which translate into three-dimensional measurements in space, and are arranged in three layers in the barrel and two layers in the forward regions. The silicon strip detector is composed of 10 layers in the barrel region, four of which are double-sided, and 12 layers in the endcap, where three out of six rings are with double-sided modules. Precise determination of the position of all silicon modules (alignment) is one of the critical aspects for achieving the designed 
resolutions of muon track parameters and is an important element of this analysis [30]. The muon system has detection planes composed of three distinct detector technologies installed outside the solenoid and embedded in the steel return yoke: drift tubes (in the barrel, $|\eta|<1.2$ ), cathode strip chambers (in the endcaps, $0.9<|\eta|<2.5$ ), and resistive plate chambers (in both barrel and endcap regions, $|\eta|<1.6)$ [29].

This analysis uses data from proton-proton collisions at $\sqrt{s}=7 \mathrm{TeV}$ collected during 2010 and 2011, and corresponding to $(1.07 \pm 0.05) \mathrm{fb}^{-1}$ of integrated luminosity. The signal and background processes $q \bar{q} \rightarrow \gamma^{*} / Z \rightarrow$ $\mu^{-} \mu^{+}$and $\tau^{-} \tau^{+}$have been simulated with the NLO QCD generator POWHEG. Parton showering is simulated using PYTHIA. The NLO PDFs used are CT10 [20]. Background samples of $W+$ jets and $t \bar{t}$ are generated using MADGRAPH [31], PYTHIA, and TAUOLA [32]. Backgrounds from $W W, W Z, Z Z$, and QCD are generated using PYTHIA. Generated events are processed through the CMS detector simulation and reconstruction. The detector simulation is based on GEANT4 $[33,34]$.

Muon candidates are selected from a sample triggered online by events with at least two muons within the volume defined by $|\eta|<2.4$ and with transverse momentum $\left(p_{T}\right)$ requirements. These requirements depend on the period of data-taking; however, they always accept two muons with $p_{T}$ of at least 8 and $13 \mathrm{GeV}$, respectively. Offline, muon tracks are first reconstructed independently in the tracker and the muon system. Muon candidates are then reconstructed by two different algorithms [35]. The global muon algorithm matches tracks in the tracker to tracks in the muon system, and then refits the individual hits in the tracker and muon system to one overall track. The tracker muon algorithm extrapolates tracks in the tracker with $p_{T}>0.5 \mathrm{GeV}$ and $p>2.5 \mathrm{GeV}$ to the muon system, and a track is taken to be a muon candidate if it matches at least one track segment in the muon system. Both algorithms take into account energy loss and multiple scattering in the steel yoke of the CMS magnet. Selection criteria demand at least 10 hits in the tracker, including one in the pixel detector, at least one hit in the muon system, and a normalized $\chi^{2}<10$ for the global fit.

Muons are required to have a small impact parameter, less than $2 \mathrm{~mm}$ measured with respect to the beam spot in the plane perpendicular to the beam direction. This requirement removes cosmic-ray muons and background events with displaced vertices. We further require the angle between the two muon tracks to be larger than $2.5 \mathrm{mrad}$ in the laboratory frame when the direction of one of the tracks is reversed. This removes any remaining cosmic-ray background and has negligible effect on the signal. To isolate single muons from muons overlapping with jets, the sum of the transverse momentum of tracks in the tracker (excluding the muon in question) within a surrounding cone of $\Delta R \equiv \sqrt{(\Delta \eta)^{2}+(\Delta \phi)^{2}}<0.3$ is required to be less than
$15 \%$ of the measured transverse momentum of the muon, where $\Delta \eta$ and $\Delta \phi$ are the differences in pseudorapidity and in azimuthal angle in radians between the muon and the track. The ECAL and HCAL are not used for muon isolation, to reduce the effect from FSR and to maximize the amount of signal events.

The kinematic requirements in the laboratory frame are $|\eta|<2.4$ and $p_{T}>18$ and $8 \mathrm{GeV}$ for the two muons. We introduce additional requirements in the Collins-Soper frame in order to simplify the acceptance parameterization: $\left|\eta^{*}\right|<2.3$ and $p_{T}^{*}>18 \mathrm{GeV}$, where $\eta^{*}$ and $p_{T}^{*}$ are defined with respect to the $z^{\prime}$-axis, described previously. We also require the dimuon transverse momentum in the laboratory frame to be less than $25 \mathrm{GeV}$ in order to suppress the contribution of events with hard jet radiation. Dilepton events are selected from events containing two oppositely charged, isolated, high- $p_{T}$ muons with a dilepton invariant mass $m$ in the range $80-100 \mathrm{GeV}$. The dimuon rapidity $Y$ is calculated from the lepton four-momenta as shown in Eq. (5). Restrictions on $\theta^{*}$ and $Y$ are motivated by detector acceptance effects, as discussed in Sec. IV. The number of selected events in the data is $N=297364$.

\section{ANALYSIS METHOD}

We use an unbinned extended maximum-likelihood fit that simultaneously describes the signal and background yields and the parameters of the $\left(Y, \hat{s}, \cos \theta^{*}\right)$ distributions. The likelihood function is written as

$$
\begin{aligned}
\mathcal{L}= & \exp \left(-n_{\mathrm{sig}}-n_{\mathrm{bkg}}\right) \prod_{i}^{N}\left(n_{\mathrm{sig}} \times \mathcal{P}_{\mathrm{sig}}\left(\vec{x}_{i} ; \theta_{\mathrm{eff}} ; \vec{\xi}\right)\right. \\
& \left.+n_{\mathrm{bkg}} \times \mathcal{P}_{\mathrm{bkg}}\left(\vec{x}_{i} ; \vec{\xi}\right)\right),
\end{aligned}
$$

where each event candidate $i$ is characterized by a set of three observables $\vec{x}_{i}=\left\{Y, \hat{s}, \cos \theta^{*}\right\}_{i}, n_{\text {sig }}$ is the number of signal events, which includes all intermediate states $\left(\gamma^{*}, Z\right.$, and their interference), $n_{\mathrm{bkg}}$ is the small number of background events, $\mathcal{P}_{\text {sig }}\left(\vec{x}_{i} ; \vec{\xi}\right)$ and $\mathcal{P}_{\text {bkg }}\left(\vec{x}_{i} ; \vec{\xi}\right)$ are the probability density functions for signal and background processes, and $\vec{\xi}$ represent the parameters of these functions. The signal probability density function is defined as

$$
\begin{aligned}
& \mathcal{P}_{\text {sig }}\left(Y, \hat{s}, \cos \theta^{*} ; \theta_{\text {eff }}\right)=G\left(Y, \hat{s}, \cos \theta^{*}\right) \\
& \quad \times \int_{-\infty}^{+\infty} d x \mathcal{R}(x) \mathcal{P}_{\text {ideal }}\left(Y, \hat{s}-x, \cos \theta^{*} ; \theta_{\text {eff }}\right) .
\end{aligned}
$$

The ideal distribution $\mathcal{P}_{\text {ideal }}\left(Y, \hat{s}, \cos \theta^{*} ; \theta_{\text {eff }}\right)$ in Eq. (13) is the Drell-Yan cross section defined in Eq. (7). We correct $\mathcal{P}_{\text {ideal }}$ for detector effects, such as acceptance, parameterized with $G\left(Y, \hat{s}, \cos \theta^{*}\right)$, and resolution and photon emission (FSR), parameterized with $\mathcal{R}(x)$ where $x$ is the change in the dilepton center-of-mass energy squared.

The acceptance function $G\left(Y, \hat{s}, \cos \theta^{*}\right)$ describes the nonuniform reconstruction efficiency as a function of the 
three observables, which includes effects from online trigger requirements, detector acceptance, reconstruction algorithms, and selection requirements. The most important effect is the loss of particles near the beam directions and the second-most-important effect is the minimum transverse momentum requirement on the leptons. Otherwise, the efficiency across the acceptance range, defined by the selection requirements $\left|\eta^{*}\right|<Y_{\max }=2.3$ and $p_{T}^{*}>p_{\min }=18 \mathrm{GeV}$, is close to uniform. The above selection requirements define a sharp boundary in $\left(Y, \hat{s}, \cos \theta^{*}\right)$ space, which can be expressed as limits on $\cos \theta^{*}$ for given $Y$ and $\hat{s}$ values as follows:

$$
\begin{gathered}
\left|\cos \theta^{*}\right|<\tanh \left(Y_{\max }-|Y|\right), \\
\left|\cos \theta^{*}\right|<\sqrt{1-4 p_{\min }^{2} / \hat{s}} .
\end{gathered}
$$

This boundary is illustrated in Fig. 6 in the $\left(Y, \cos \theta^{*}\right)$ plane for a fixed value $\hat{s}=m_{Z}^{2}$.

The effect of smearing the muon track parameters, such as the muon momentum and direction, due to detector resolution and FSR, is most evident in the invariant mass distribution. This effect is parameterized with the function $\mathcal{R}(x)$ in Eq. (13). Both acceptance and resolution effects are illustrated in Fig. 7, where the analytical parameterization of $Y, m=\sqrt{\hat{s}}$, and $\cos \theta^{*}$ is in good agreement with LO simulation in both QCD and EWK, as generated by PYTHIA. Although a wider $m$ range is investigated, the analysis is performed in the range $80<m<100 \mathrm{GeV}$ to reduce uncertainties from FSR. In this illustration, FSR is included and the major detector effects are introduced in the following way: for the three track parameters $\left(p_{T}, \phi\right.$, $\theta$ ), we apply Gaussian random smearing with standard deviation of $\Delta p_{T}=0.025 p_{T}+0.0001 p_{T}^{2}$ (with $p_{T}$ in

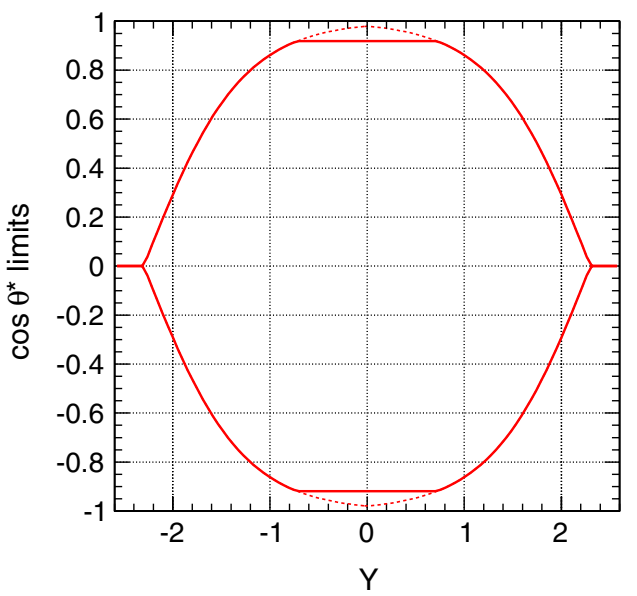

FIG. 6 (color online). Accepted $\cos \theta^{*}$ range as a function of $Y$ for $\hat{s}=m_{Z}^{2}$ and for the kinematic selection used in this analysis. The outer boundary corresponds to Eq. (14) and the horizontal lines near $\cos \theta^{*}= \pm 0.92$ correspond to Eq. (15).
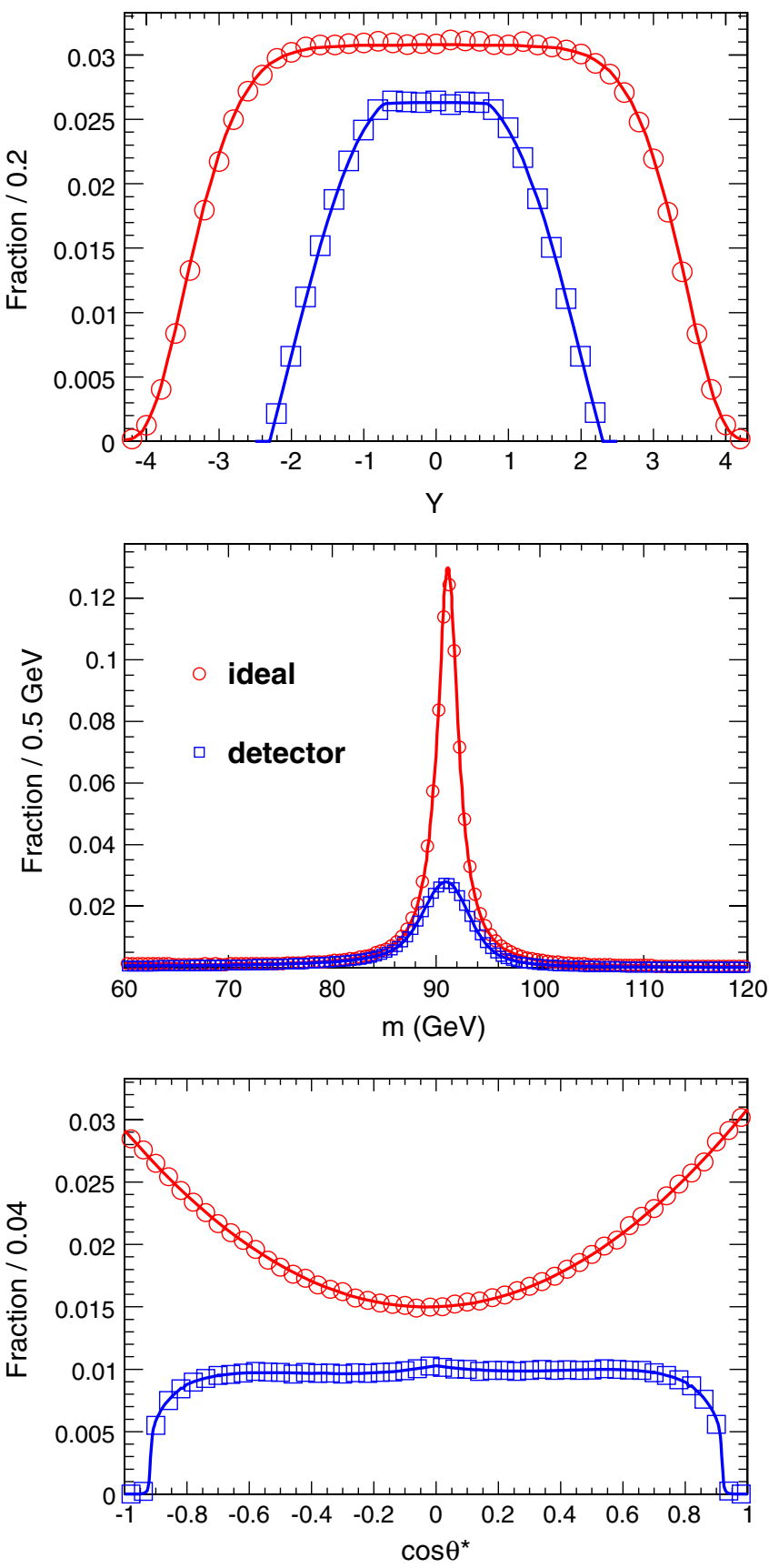

FIG. 7 (color online). Distributions of $Y$ (top), $m$ (middle), and $\cos \theta^{*}$ (bottom), from PYTHIA simulation (points) of the $q \bar{q} \rightarrow$ $\gamma^{*} / Z \rightarrow \mu^{-} \mu^{+}$process and its analytical parameterization (smooth curve). Combined distributions from Fig. 5 appear in red at the top of each plot (red circles for an "ideal" simulation), while distributions after acceptance and resolution effects, including photon FSR, appear in blue below (blue squares for a simplified "detector" simulation). Distributions are normalized to unit area and are shown as fractions of events per bin.

$\mathrm{GeV}), \Delta \phi=\Delta \theta=0.001 \mathrm{rad}$, and neglect resolution effects on the track origin. This simplified simulation of detector effects is found useful to isolate production model uncertainties from the detector effect parameterization. 
Further studies are performed with full GEANT4-based modeling of the CMS detector using the POWHEG simulation of the dimuon events and with PYTHIA simulation of the parton showering and FSR. In the parameterization of the acceptance function $\mathcal{G}\left(Y, \hat{s}, \cos \theta^{*}\right)$, we model the small deviations from a uniform efficiency with empirical polynomial functions that include correlations of the two observables within the boundaries of the $\left(Y, \cos \theta^{*}\right)$ plane defined above. This efficiency parameterization is derived from the simulation with a fit where the parameters of the polynomial functions are left unconstrained. The main effect is a loss of efficiency in the vicinity of the acceptance boundaries. A similar approach is later employed as part of the systematic uncertainty studies where the parameters of the efficiency model are left free in the fit to data.

In the parameterization of the resolution function $\mathcal{R}(x)$, FSR is modeled with PYTHIA and resolution effects are taken from the full CMS detector simulation, including the effects of tracker alignment on the tracking resolution. The function $\mathcal{R}(x)$ is approximated with a sum of four Gaussian functions, to allow for the analytical convolution in Eq. (13) and be flexible enough to describe both detector resolution and FSR effects. Parameters of the $\mathcal{R}(x)$ function are left free in the fit to the simulated MC sample. The overall shift of the $Z$ mass in the resolution function $\mathcal{R}(x)$ is left free in the fit to data, effectively allowing the energy scale to be determined from the data.

The background contribution is estimated by MC simulation; the QCD component has been cross-checked with data. The total expected background is about $0.05 \%$ of the signal yield. The background consists of the crossfeed from the $q \bar{q} \rightarrow Z / \gamma^{*} \rightarrow \tau^{+} \tau^{-}$process, QCD, $t \bar{t}$, and diboson production in nearly equal contributions. The probability density function for the background $\mathcal{P}_{\text {bkg }}\left(\vec{x}_{i} ; \vec{\xi}\right)$ is parameterized in a similar manner to that shown in Eq. (13) with an acceptance range defined by Eqs. (14) and (15) and the distributions within the acceptance boundaries parameterized with an empirical polynomial function. The number of background events $n_{\mathrm{bkg}}$ is fixed to the expected value of 157 events.

In Fig. 8 we show the $\cos \theta^{*}$ distributions in the data separately for the $|Y|<1$ and $|Y|>1$ regions, and compare them to the POWHEG-based simulation of the $q \bar{q} \rightarrow \gamma^{*} / Z \rightarrow \mu^{-} \mu^{+}$process in the CMS detector. Together with Fig. 4, these distributions illustrate the challenge of analyzing Drell-Yan events at the LHC. While the acceptance effects on the $\cos \theta^{*}$ distribution are moderate for smaller values of $|Y|$, the dilution is strong, as shown in Fig. 4. In contrast, the larger values of $|Y|$ have a smaller dilution effect, but the $\cos \theta^{*}$ range is strongly truncated because of the limited acceptance, as shown in Fig. 6. Therefore, the optimal analysis of the angular distributions requires proper accounting for such correlations among the three observables. At the same time, Fig. 8 shows good agreement between the data and MC simulation. Residual
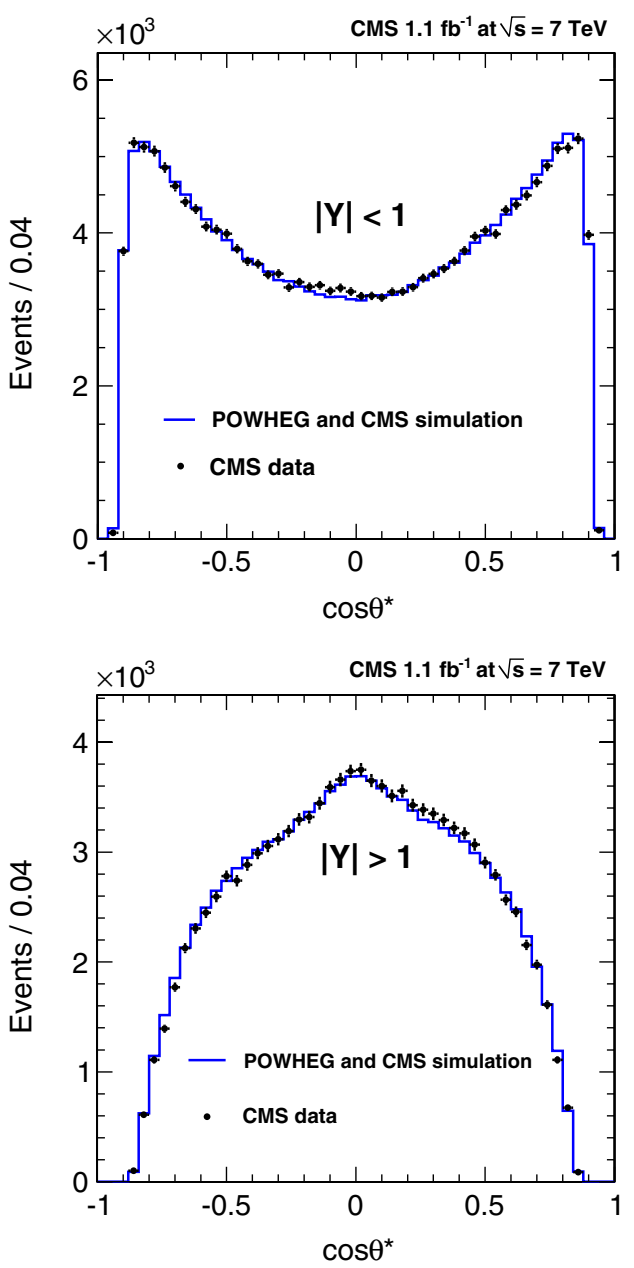

FIG. 8 (color online). Distribution of $\cos \theta^{*}$ for data (points) and POWHEG-based detector simulation with $\sin ^{2} \theta_{W}=0.2311$ (histogram) of the $q \bar{q} \rightarrow \gamma^{*} / Z \rightarrow \mu^{-} \mu^{+}$process for $|Y|<1$ (top) and $|Y|>1$ (bottom).

differences in the distributions can be explained by the somewhat different value of $\sin ^{2} \theta_{\text {eff }}=0.2311$ used in the simulation compared to the best value describing the data, and by several systematic effects accounted for below, such as the tracker misalignment, the momentum scale in the track reconstruction, and FSR modeling.

A "blind" analysis of the data is performed, in which the fit result is not examined until a review of the entire analysis is complete, including the evaluation of all associated systematic uncertainties. However, while the analysis is performed "blind," the quality of the fits to the MC simulation and data is examined. We test the performance of the fitting procedure using samples generated using Monte Carlo simulations, with each separate sample containing the same number of events observed in the data. Signal events are generated with the POWHEG-based CMS detector simulation with an input value of $\sin ^{2} \theta_{\text {eff }}=$ 0.2311. The number of background events is Poisson 

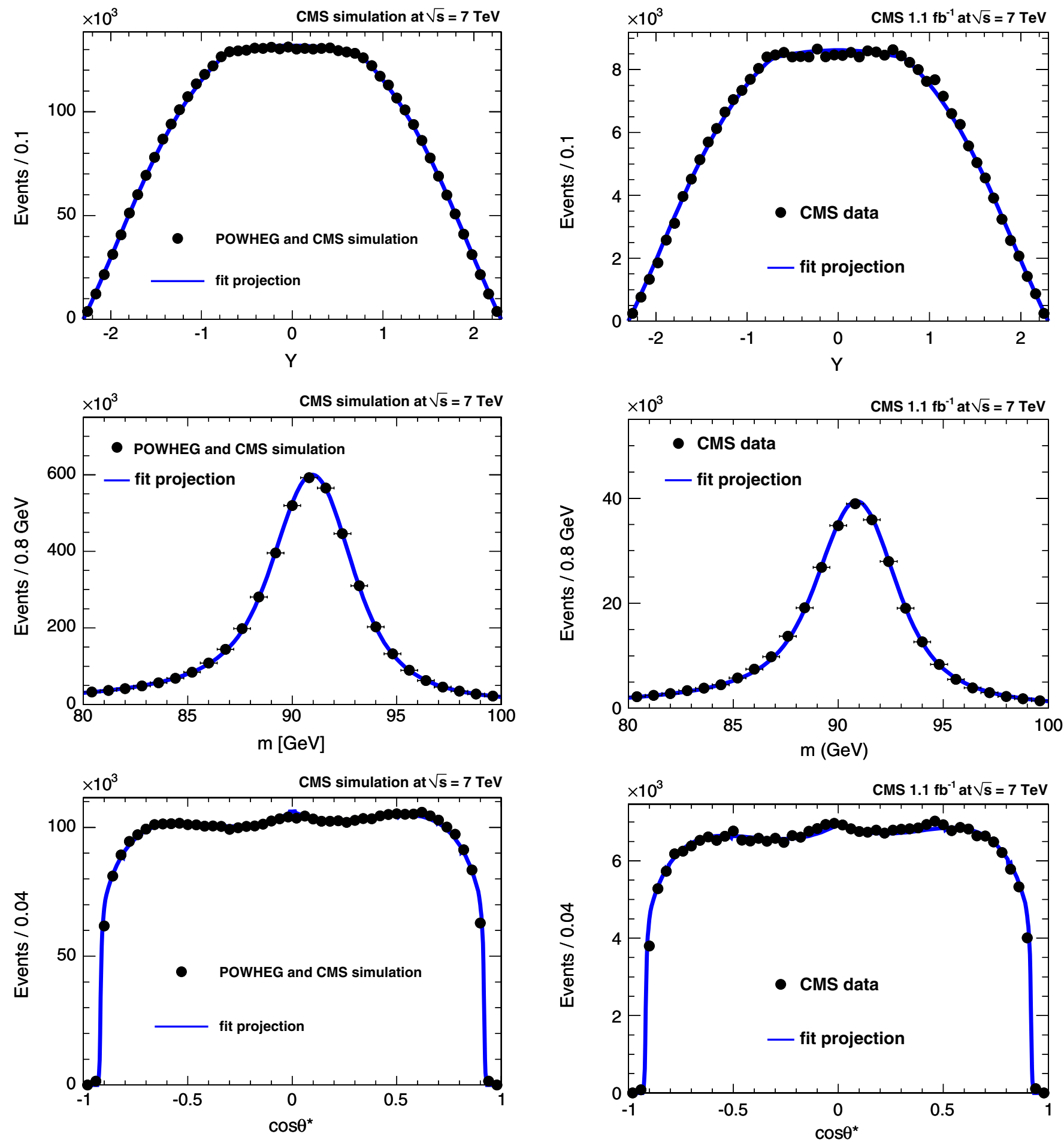

FIG. 9 (color online). Distributions of $Y$ (top), $m$ (middle), and $\cos \theta^{*}$ (bottom) from the POWHEG-based detector simulation with $\sin ^{2} \theta_{W}=0.2311$ of the $q \bar{q} \rightarrow \gamma^{*} / Z \rightarrow \mu^{-} \mu^{+}$process (points). The MC sample corresponds to an integrated luminosity of $16 \mathrm{fb}^{-1}$. The lines show the projections of the probability density functions.

distributed according to expectation. After the corrections discussed below are applied, the pull distribution is in agreement with a unit-width Gaussian distribution centered at zero. A comparison of the MC sample

FIG. 10 (color online). Distributions of $Y$ (top), $m$ (middle), and $\cos \theta^{*}$ (bottom) in the analysis of $q \bar{q} \rightarrow \gamma^{*} / Z \rightarrow \mu^{-} \mu^{+}$ events from CMS (points). The lines show the projections of the probability density functions.

projections and the probability density functions are shown in Fig. 9.

We examine the quality of the fit to the data by comparing the data distributions to the likelihood model 
expectations, and comparing the fit likelihood value $\mathcal{L}$ and the observed statistical uncertainty to those expected with the generated samples. Projections of the data and the probability density functions are shown in Fig. 10. They exhibit similar agreement as with the simulation shown in Fig. 9. Correction for the energy scale is already included in the fit model. The observed statistical error on $\sin ^{2} \theta_{\text {eff }}$ of \pm 0.0020 is in good agreement with what is expected from the MC samples discussed above. We find only small differences when comparing the likelihood value $\mathcal{L}$ for generated experiments from the likelihood model, the POWHEG-based CMS detector simulation, and the data. The level of agreement is consistent with typical differences due to imperfect efficiency function modeling and NLO effects discussed below. The variations do not affect the result of the analysis within the systematic uncertainties assigned.

\section{SYSTEMATIC UNCERTAINTIES}

The list of systematic uncertainties on the measurement of $\sin ^{2} \theta_{\text {eff }}$ and associated corrections to the fit values, as discussed below, is shown in Table II. These uncertainties arise from both theoretical assumptions and detector modeling.

We follow the PDF4LHC working group's recommendation [23] in estimating uncertainties from the PDFs. We reweight a large MC sample generated with CT10 [20] PDFs to obtain samples equivalent to MSTW 2008 [21] and NNPDF 2.1 [22] PDFs. We vary the internal degrees of freedom of the PDFs for all three sets of models. We also use this technique to vary $\alpha_{s}$, but find its uncertainties to have negligible effects compared to any of the PDF variations. We find a change in the value of $\sin ^{2} \theta_{\text {eff }}$ of $+4.8 \times 10^{-4}$ and $+3.4 \times 10^{-4}$ in using the PDFs from MSTW and NNPDF, respectively. The variations in the value of $\sin ^{2} \theta_{\text {eff }}$ within each MC simulation due to the PDF uncertainties are ${ }_{-12.1}^{+13.0} \times 10^{-4},{ }_{-4.1}^{+3.9} \times 10^{-4}$, and $\pm 7.3 \times 10^{-4}$ for CT10, MSTW, and NNPDF, respectively. The envelope of the above variations corresponds to the total systematic uncertainty of \pm 0.0013 .

TABLE II. Corrections to the fit values and systematic uncertainties in the measurement of $\sin ^{2} \theta_{\text {eff }}$.

\begin{tabular}{lcc}
\hline \hline source & correction & uncertainty \\
\hline PDF & - & \pm 0.0013 \\
FSR & - & \pm 0.0011 \\
LO model (EWK) & - & \pm 0.0002 \\
LO model (QCD) & +0.0012 & \pm 0.0012 \\
resolution and alignment & +0.0007 & \pm 0.0013 \\
efficiency and acceptance & - & \pm 0.0003 \\
background & - & \pm 0.0001 \\
\hline total & +0.0019 & \pm 0.0025 \\
\hline \hline
\end{tabular}

The FSR is modeled with PYTHIA in the parameterization of the resolution function $\mathcal{R}(x)$. As a cross-check, we use four alternative FSR models for generation, with simplified detector simulation discussed above: PYTHIA, PHOTOS [36], and two different modes in HORACE [37]. All three generator programs perform $O(\alpha)$ calculations of FSR and provide similar results, leading to differences in the fitted values of $\sin ^{2} \theta_{\text {eff }}$ of about 0.001 . In addition, the HORACE generator allows the exact $O(\alpha)$ calculation and multiple-photon radiation from all charged states, which is the state-of-the-art EWK calculation. We found that this has a larger effect on the analysis when a wide range of values for the dimuon invariant mass $m$ is used. However, with the mass range $80<m<100 \mathrm{GeV}$, the differences in the relevant part of the radiative tail become small. We perform cross-fits of the four generated samples and the four corresponding resolution functions $\mathcal{R}(x)$, finding differences in the fitted $\sin ^{2} \theta_{\text {eff }}$ values of at most 0.0011 . The PYTHIA sample typically results in larger differences from the other generators, and the assigned systematic uncertainty of \pm 0.0011 covers these deviations. The assigned error conservatively covers the FSR uncertainty in the measurement when the fraction of FSR radiation is reweighted in simulation. This reweighting technique is based on the comparison of the FSR fractions between data and the PYTHIA simulation.

Effects from NLO EWK contributions are expected to be small compared to the statistical precision of our measurement. Calculations with the ZFITTER [38] program indicate that the variation of the effective value of $\sin ^{2} \theta_{\text {eff }}$ for light quarks is within 0.0002 of the lepton values. It is only the heavier $b$ quark that requires corrections of the order 0.001. However, given that only about $2.5 \%$ of the dileptons are produced in $b \bar{b}$ interactions, and no angular asymmetry can be measured because of the dilution factor with this initial state, these corrections have a negligible effect on our measurements.

Parameterization of the likelihood function models both the initial-state interactions and the PDFs at LO. The requirement that the dimuon transverse momentum $p_{T}$ be less than $25 \mathrm{GeV}$ suppresses the contribution of events with hard jet radiation and reduces the effects from NLO processes. This requirement also ensures that the transformation between the laboratory frame and the Collins-Soper frame is small, and that our analytical description of acceptance effects is correct, without any loss of acceptance coverage. With generated samples, we observe a bias of $-0.0012 \pm 0.0006$ in the fit value of $\sin ^{2} \theta_{\text {eff }}$, which is attributed to NLO effects. In this test, perfect CMS detector conditions are simulated, which removes most of the detector effects discussed below. To be conservative, we apply a correction of +0.0012 and assign a systematic uncertainty of \pm 0.0012 to cover all effects associated with the LO model. For example, we have investigated 
the dependence of the expected and observed shift in the fit result as a function of the $p_{T}$ requirement over a wide range of $p_{T}$. The results are stable within the uncertainty quoted. The distribution of $p_{T}$ exhibits only a small difference between the data and the MC simulation. We reweight the MC simulation to match the $p_{T}$ distribution to the data and observe that the results of the fit to the reweighted $\mathrm{MC}$ events are consistent with the case without reweighting to within 0.0004 . Treating the correction as an additive or multiplicative factor does not affect the final result, as long as the observed value of $\sin ^{2} \theta_{\text {eff }}$ is close to what is expected.

The detector resolution in the muon track reconstruction is affected by contributions from the silicon tracker alignment. We perform a realistic simulation of the alignment procedure to model the statistical precision of the track reconstruction. We observe a bias of -0.0013 in the fit result of $\sin ^{2} \theta_{\text {eff }}$ when the realistic simulation of alignment is used in place of perfect conditions. We find that while the statistical precision of the track reconstruction is well modeled by the realistic simulation, the biases from $\chi^{2}$-invariant detector deformations [30] may not necessarily be well reproduced in the MC simulation. We have investigated nine basic distortions in the tracker reconstruction geometry, which follow from the cylindrical symmetry of the system [30,39]. In each case, the procedure of the tracker alignment is repeated after the distortion is introduced. The effects of the remaining distortions on $\sin ^{2} \theta_{\text {eff }}$ are all smaller than 0.001. The typical initial distortions are taken to be $200 \mu \mathrm{m}$, which is the approximate value of the constraints from the detector survey, the placement tolerance, and the observed agreement in the alignment procedure.

In the end, constraints on the above distortions in the tracker reconstruction geometry come from the data. We have observed that distortions affecting the $\sin ^{2} \theta_{\text {eff }}$ fit values also introduce a bias in the mass of the dimuon pair $m$ as a function of $\cos \theta^{*}$. We observe a linear trend in the bias of the average value of $m$ as a function of $\cos \theta^{*}$, with a slope of $-0.072 \mathrm{GeV}$, in the realistic simulation that is twice as large as that observed in data, $-0.039 \mathrm{GeV}$, when both are compared to simulation with the ideal geometry model. We also observe a bias in the value of $\sin ^{2} \theta_{\text {eff }}$ that is twice as large when an additional systematic distortion is introduced in the realistic simulation, resulting in the slope of the average $m$ value versus $\cos \theta^{*}$ dependence also becoming twice as large. From these studies, we assign a correction of +0.0007 to the fit value of $\sin ^{2} \theta_{\text {eff }}$ due to alignment effects and a systematic uncertainty of \pm 0.0013 to cover the range of possible deviations observed. In order to minimize the uncertainties from the energy scale bias in the track reconstruction, the shift of the $Z$ mass in the resolution function $\mathcal{R}(x)$ is left free in the fit, effectively allowing the energy scale to be determined from the fit to the data. Consistency between the fit value from the data and the expectation from the MC simulation is found to be within $0.1 \mathrm{GeV}$.

We find very weak sensitivity to the efficiency parameterization $\mathcal{G}\left(Y, \hat{s}, \cos \theta^{*}\right)$ across the acceptance range because the efficiency is symmetric in $\cos \theta^{*}$. This leads to negligible effects on the odd terms in the angular distribution that are sensitive to $\sin ^{2} \theta_{\text {eff }}$. The sign of $Y$ is defined by the dimuon system direction along the counterclockwise beam and has no preferred direction. The sign of $\cos \theta^{*}$ is defined by the charge of the "forward" lepton. The cylindrical symmetry of CMS, combined with the random nature of the forward direction, leads to a symmetry in the efficiency function. This has been verified with a detailed GEANT4-based simulation of the CMS detector, including calibration and alignment effects. Even in the extreme case of $G\left(Y, \hat{s}, \cos \theta^{*}\right)$ being flat across the acceptance range, negligible changes in the fit results are observed with simulated samples. We also allow parameters of the model to be free in the fit to data. We assign a systematic uncertainty of 0.0003 due to efficiency and acceptance parameterization, which is the level of consistency of results from these studies.

The number of background events $n_{\mathrm{bkg}}$ is fixed to the expected value and is varied according to its associated uncertainties. We assign a 50\% uncertainty to the QCD rate, based on studies with wrong-sign lepton pairs. The relative size of the sum of the EWK background processes is expected to be reproduced by simulation to a precision of better than $20 \%$. However, in the mass range $80<m<$ $100 \mathrm{GeV}$, the fraction of background is only $0.05 \%$, and the fit results are insensitive to the exact treatment of the background. The measured $\sin ^{2} \theta_{\text {eff }}$ value remains stable within 0.0001 , even when the background is removed from the model.

\section{RESULTS AND DISCUSSION}

We have presented a likelihood method to analyze the Drell-Yan process at the LHC. The process is described by the correlated dilepton rapidity, invariant mass, and decay angle distributions. The quark direction in the elementary parton collisions, which is not directly accessible in the proton-proton collisions at the LHC, is modeled statistically using correlations between the observables. The result of the analysis, which includes systematic uncertainties and corrections from Table II, is

$$
\sin ^{2} \theta_{\text {eff }}=0.2287 \pm 0.0020 \text { (stat.) } \pm 0.0025 \text { (syst.). }
$$

This measurement of the effective weak mixing angle in the predominantly $u \bar{u}, d \bar{d} \rightarrow \gamma^{*} / Z \rightarrow \mu^{-} \mu^{+}$processes in proton-proton collisions is consistent with measurements in other processes [5,7-11], as expected within the standard model.

The dominant systematic uncertainties in the measurement include modeling of the PDFs, FSR, effects beyond the leading order in QCD, as well as detector uncertainties 
primarily due to tracker alignment. With increased statistics of the Drell-Yan process at the LHC, a further reduction of the systematic uncertainties will become critical. Understanding the tracker alignment will certainly improve as the collaboration gains further experience. Therefore, we expect the limiting uncertainties to come from the Drell-Yan process modeling.

Uncertainties from PDFs will decrease as better constraints on the proton model become available from the LHC and elsewhere. In fact, the Drell-Yan process is itself a useful input to the PDF model constraints, and the methods discussed in this paper can be used to constrain the parameters in the PDF model. However, one must be careful not to mix information used for PDF constraints from the Drell-Yan process with measurements using the same events, unless the correlations are properly taken into account. Uncertainties from the FSR model may be improved as higher-order electroweak calculations are integrated with the higher-order QCD calculations of the matrix element in the Drell-Yan process, such as the incorporation of POWHEG and HORACE.

The LO approximations in the model may be further improved as NLO matrix elements are employed in the likelihood approach and more variables are integrated into the analysis. We view the current LO formalism as a conceptual step in developing multivariate matrix-element approaches to resonance polarization analyses, which can be applied to precision measurements, as well as potential new resonances that may be discovered at the LHC. The evolution of this method may also allow several parameters of the electroweak couplings to be determined simultaneously, such as a measurement of the vector and axialvector couplings of the light quarks separately from the lepton couplings.

\section{ACKNOWLEDGMENTS}

We would like to thank Kirill Melnikov and Alessandro Vicini for useful discussions of NLO QCD and EWK effects. We wish to congratulate our colleagues in the CERN accelerator departments for the excellent performance of the LHC machine. We thank the technical and administrative staff at CERN and other CMS institutes. This work was supported by the Austrian Federal Ministry of Science and Research; the Belgium Fonds de la Recherche Scientifique, and Fonds voor Wetenschappelijk Onderzoek; the Brazilian Funding Agencies (CNPq, CAPES, FAPERJ, and FAPESP); the Bulgarian Ministry of Education and Science; CERN; the Chinese Academy of Sciences, Ministry of Science and Technology, and National Natural Science
Foundation of China; the Colombian Funding Agency (COLCIENCIAS); the Croatian Ministry of Science, Education and Sport; the Research Promotion Foundation, Cyprus; the Estonian Academy of Sciences and NICPB; the Academy of Finland, Finnish Ministry of Education and Culture, and Helsinki Institute of Physics; the Institut National de Physique Nucléaire et de Physique des Particules/CNRS, and Commissariat à l'Énergie Atomique et aux Énergies Alternatives/CEA, France; the Bundesministerium für Bildung und Forschung, Deutsche Forschungsgemeinschaft, and Helmholtz-Gemeinschaft Deutscher Forschungszentren, Germany; the General Secretariat for Research and Technology, Greece; the National Scientific Research Foundation, and National Office for Research and Technology, Hungary; the Department of Atomic Energy and the Department of Science and Technology, India; the Institute for Studies in Theoretical Physics and Mathematics, Iran; the Science Foundation, Ireland; the Istituto Nazionale di Fisica Nucleare, Italy; the Korean Ministry of Education, Science and Technology and the World Class University program of NRF, Korea; the Lithuanian Academy of Sciences; the Mexican Funding Agencies (CINVESTAV, CONACYT, SEP, and UASLP-FAI); the Ministry of Science and Innovation, New Zealand; the Pakistan Atomic Energy Commission; the State Commission for Scientific Research, Poland; the Fundação para a Ciência e a Tecnologia, Portugal; JINR (Armenia, Belarus, Georgia, Ukraine, Uzbekistan); the Ministry of Science and Technologies of the Russian Federation, the Russian Ministry of Atomic Energy and the Russian Foundation for Basic Research; the Ministry of Science and Technological Development of Serbia; the Ministerio de Ciencia e Innovación, and Programa Consolider-Ingenio 2010, Spain; the Swiss Funding Agencies (ETH Board, ETH Zurich, PSI, SNF, UniZH, Canton Zurich, and SER); the National Science Council, Taipei; the Scientific and Technical Research Council of Turkey, and Turkish Atomic Energy Authority; the Science and Technology Facilities Council, UK; the US Department of Energy, and the US National Science Foundation. Individuals have received support from the Marie-Curie programme and the European Research Council (European Union); the Leventis Foundation; the A. P. Sloan Foundation; the Alexander von Humboldt Foundation; the Belgian Federal Science Policy Office; the Fonds pour la Formation à la Recherche dans l'Industrie et dans l'Agriculture (FRIA-Belgium); the Agentschap voor Innovatie door Wetenschap en Technologie (IWT-Belgium); and the Council of Science and Industrial Research, India. 
[1] L. Evans and P. Bryant, JINST 3, S08001 (2008).

[2] S. Drell and T.-M. Yan, Phys. Rev. Lett. 25, 316 (1970).

[3] D. London and J. L. Rosner, Phys. Rev. D 34, 1530 (1986).

[4] J. L. Rosner, Phys. Rev. D 35, 2244 (1987).

[5] ALEPH Collaboration, DELPHI Collaboration, L3 Collaboration, OPAL Collaboration, SLD Collaboration, LEP Electroweak Working Group, SLD Electroweak Group, and SLD Heavy Flavour Group, Phys. Rep. 427, 257 (2006).

[6] S. Weinberg, Phys. Rev. Lett. 19, 1264 (1967).

[7] G. Zeller et al. (NuTeV), Phys. Rev. Lett. 88, 091802 (2002).

[8] D. Acosta et al. (CDF), Phys. Rev. D 71, 052002 (2005).

[9] V. Abazov et al. (D0), Phys. Rev. Lett. 101, 191801 (2008).

[10] V. Abazov et al. (D0), Phys. Rev. D 84, 012007 (2011).

[11] A. Aktas et al. (H1), Phys. Lett. B 632, 35 (2006).

[12] P. Fisher, U. Becker, and J. Kirkby, Phys. Lett. B 356, 404 (1995).

[13] M. Dittmar, Phys. Rev. D 55, 161 (1997).

[14] G. Altarelli and M. L. Mangano, Report No. CERN-2000004, http://cdsweb.cern.ch/record/425440.

[15] Y. Gao, A. V. Gritsan, Z. Guo, K. Melnikov, M. Schulze et al., Phys. Rev. D 81, 075022 (2010).

[16] K. Nakamura et al. (Particle Data Group), J. Phys. G 37, 075021 (2010).

[17] J.C. Collins and D.E. Soper, Phys. Rev. D 16, 2219 (1977).

[18] A. De Roeck and R. Thorne, Prog. Part. Nucl. Phys. 66, 727 (2011)

[19] S. Kretzer, H. Lai, F. Olness, and W. Tung, Phys. Rev. D 69, 114005 (2004).

[20] H.-L. Lai, M. Guzzi, J. Huston, Z. Li, P. M. Nadolsky et al., Phys. Rev. D 82, 074024 (2010).

[21] A. D. Martin, W. J. Stirling, R. S. Thorne, and G. Watt, Eur. Phys. J. C 63, 189 (2009).
[22] R.D. Ball, L. Del Debbio, S. Forte, A. Guffanti, J. I. Latorre et al., Nucl. Phys. B838, 136 (2010).

[23] M. Botje, J. Butterworth, A. Cooper-Sarkar, A. de Roeck, J. Feltesse et al., arXiv:1101.0538.

[24] T. Sjöstrand, S. Mrenna, and P.Z. Skands, J. High Energy Phys. 05 (2006) 026.

[25] P. Nason, J. High Energy Phys. 11 (2004) 040.

[26] S. Frixione, P. Nason, and C. Oleari, J. High Energy Phys. 11 (2007) 070.

[27] S. Alioli, P. Nason, C. Oleari, and E. Re, J. High Energy Phys. 07 (2008) 060.

[28] S. Chatrchyan et al. (CMS), JINST 3, 508004 (2008).

[29] CMS Collaboration, Tech. Rep. No. CERN-LHCC-2006001/CMS-TDR-008-1, 2006, http://cdsweb.cern.ch/ record/922757.

[30] S. Chatrchyan et al. (CMS), JINST 5, T03009 (2010).

[31] J. Alwall, P. Demin, S. de Visscher, R. Frederix, M. Herquet et al., J. High Energy Phys. 09 (2007) 028.

[32] N. Davidson, G. Nanava, T. Przedzinski, E. Richter-Was, and Z. Was, arXiv:1002.0543.

[33] S. Agostinelli et al. (GEANT4), Nucl. Instrum. Methods Phys. Res., Sect. A 506, 250 (2003).

[34] J. Allison, K. Amako, J. Apostolakis, H. Araujo, P. Dubois et al., IEEE Trans. Nucl. Sci. 53, 270 (2006).

[35] CMS Collaboration, Report No. CMS-PAS-MUO-10-002, 2010, http://cdsweb.cern.ch/record/1279140 .

[36] P. Golonka and Z. Was, Eur. Phys. J. C 45, 97 (2006).

[37] C. Carloni Calame, G. Montagna, O. Nicrosini, and A. Vicini, J. High Energy Phys. 10 (2007) 109.

[38] A. Arbuzov, M. Awramik, M. Czakon, A. Freitas, M. Grunewald et al., Comput. Phys. Commun. 174, 728 (2006).

[39] D. Brown, A. Gritsan, Z. Guo, and D. Roberts, Nucl. Instrum. Methods Phys. Res., Sect. A 603, 467 (2009).

S. Chatrchyan, ${ }^{1}$ V. Khachatryan, ${ }^{1}$ A. M. Sirunyan, ${ }^{1}$ A. Tumasyan, ${ }^{1}$ W. Adam, ${ }^{2}$ T. Bergauer, ${ }^{2}$ M. Dragicevic, ${ }^{2}$ J. Erö, ${ }^{2}$ C. Fabjan, ${ }^{2}$ M. Friedl, ${ }^{2}$ R. Frühwirth, ${ }^{2}$ V. M. Ghete, ${ }^{2}$ J. Hammer, ${ }^{2, b}$ S. Hänsel, ${ }^{2}$ M. Hoch, ${ }^{2}$ N. Hörmann, ${ }^{2}$ J. Hrubec, ${ }^{2}$ M. Jeitler, ${ }^{2}$ W. Kiesenhofer, ${ }^{2}$ M. Krammer, ${ }^{2}$ D. Liko, ${ }^{2}$ I. Mikulec, ${ }^{2}$ M. Pernicka, ${ }^{2}$ B. Rahbaran, ${ }^{2}$ H. Rohringer, ${ }^{2}$ R. Schöfbeck, ${ }^{2}$ J. Strauss, ${ }^{2}$ A. Taurok ${ }^{2}$ F. Teischinger, ${ }^{2}$ C. Trauner, ${ }^{2}$ P. Wagner, ${ }^{2}$ W. Waltenberger, ${ }^{2}$ G. Walzel, ${ }^{2}$ E. Widl, ${ }^{2}$ C.-E. Wulz, ${ }^{2}$ V. Mossolov, ${ }^{3}$ N. Shumeiko, ${ }^{3}$ J. Suarez Gonzalez, ${ }^{3}$ S. Bansal, ${ }^{4}$ L. Benucci, ${ }^{4}$ E. A. De Wolf, X. Janssen, ${ }^{4}$ S. Luyckx ${ }^{4}$ T. Maes, ${ }^{4}$ L. Mucibello, ${ }^{4}$ S. Ochesanu,${ }^{4}$ B. Roland, ${ }^{4}$ R. Rougny, ${ }^{4}$ M. Selvaggi, ${ }^{4}$ H. Van Haevermaet, ${ }^{4}$ P. Van Mechelen, ${ }^{4}$ N. Van Remortel,${ }^{4}$ F. Blekman, ${ }^{5}$ S. Blyweert, ${ }^{5}$ J. D'Hondt, ${ }^{5}$

R. Gonzalez Suarez, ${ }^{5}$ A. Kalogeropoulos, ${ }^{5}$ M. Maes, ${ }^{5}$ A. Olbrechts, ${ }^{5}$ W. Van Doninck, ${ }^{5}$ P. Van Mulders, ${ }^{5}$ G. P. Van Onsem, ${ }^{5}$ I. Villella, ${ }^{5}$ O. Charaf, ${ }^{6}$ B. Clerbaux, ${ }^{6}$ G. De Lentdecker, ${ }^{6}$ V. Dero, ${ }^{6}$ A. P. R. Gay, ${ }^{6}$ G. H. Hammad, ${ }^{6}$ T. Hreus, ${ }^{6}$ P. E. Marage, ${ }^{6}$ A. Raval, ${ }^{6}$ L. Thomas, ${ }^{6}$ G. Vander Marcken, ${ }^{6}$ C. Vander Velde, ${ }^{6}$ P. Vanlaer, ${ }^{6}$ V. Adler, ${ }^{7}$ A. Cimmino, ${ }^{7}$ S. Costantini, ${ }^{7}$ M. Grunewald, ${ }^{7}$ B. Klein, ${ }^{7}$ J. Lellouch, ${ }^{7}$ A. Marinov, ${ }^{7}$ J. Mccartin, ${ }^{7}$ D. Ryckbosch, ${ }^{7}$ F. Thyssen, ${ }^{7}$ M. Tytgat, ${ }^{7}$ L. Vanelderen, ${ }^{7}$ P. Verwilligen, ${ }^{7}$ S. Walsh,${ }^{7}$ N. Zaganidis, ${ }^{7}$ S. Basegmez, ${ }^{8}$ G. Bruno, ${ }^{8}$ J. Caudron, ${ }^{8}$ L. Ceard, ${ }^{8}$ E. Cortina Gil,${ }^{8}$ J. De Favereau De Jeneret, ${ }^{8}$ C. Delaere, ${ }^{8}$ D. Favart, ${ }^{8}$ L. Forthomme, ${ }^{8}$ A. Giammanco, ${ }^{8}$ G. Grégoire, ${ }^{8}$ J. Hollar, ${ }^{8}$ V. Lemaitre, ${ }^{8}$ J. Liao, ${ }^{8}$ O. Militaru, ${ }^{8}$ C. Nuttens, ${ }^{8}$ S. Ovyn, ${ }^{8}$ D. Pagano, ${ }^{8}$ A. Pin,${ }^{8}$ K. Piotrzkowski, ${ }^{8}$ N. Schul, ${ }^{8}$ N. Beliy, ${ }^{9}$ T. Caebergs, ${ }^{9}$ E. Daubie,,${ }^{9}$ G. A. Alves,${ }^{10}$ L. Brito, ${ }^{10}$

D. De Jesus Damiao, ${ }^{10}$ M.E. Pol, ${ }^{10}$ M. H. G. Souza, ${ }^{10}$ W. L. Aldá Júnior,${ }^{11}$ W. Carvalho, ${ }^{11}$ E. M. Da Costa,${ }^{11}$ C. De Oliveira Martins, ${ }^{11}$ S. Fonseca De Souza, ${ }^{11}$ D. Matos Figueiredo, ${ }^{11}$ L. Mundim, ${ }^{11}$ H. Nogima, ${ }^{11}$ V. Oguri, ${ }^{11}$ W. L. Prado Da Silva, ${ }^{11}$ A. Santoro, ${ }^{11}$ S. M. Silva Do Amaral, ${ }^{11}$ A. Sznajder, ${ }^{11}$ T. S. Anjos, ${ }^{12, \mathrm{c}}$ C. A. Bernardes, ${ }^{12, \mathrm{c}}$ 
F. A. Dias, ${ }^{12, \mathrm{~d}}$ T. R. Fernandez Perez Tomei, ${ }^{12}$ E. M. Gregores, ${ }^{12, \mathrm{c}}$ C. Lagana, ${ }^{12}$ F. Marinho, ${ }^{12}$ P. G. Mercadante, ${ }^{12, \mathrm{c}}$ S. F. Novaes, ${ }^{12}$ Sandra S. Padula,${ }^{12}$ N. Darmenov,${ }^{13, b}$ V. Genchev, ${ }^{13, b}$ P. Iaydjiev, ${ }^{13, b}$ S. Piperov, ${ }^{13}$ M. Rodozov,${ }^{13}$ S. Stoykova, ${ }^{13}$ G. Sultanov, ${ }^{13}$ V. Tcholakov, ${ }^{13}$ R. Trayanov, ${ }^{13}$ M. Vutova, ${ }^{13}$ A. Dimitrov,${ }^{14}$ R. Hadjiiska, ${ }^{14}$ A. Karadzhinova, ${ }^{14}$ V. Kozhuharov,${ }^{14}$ L. Litov, ${ }^{14}$ M. Mateev,${ }^{14}$ B. Pavlov ${ }^{14}$ P. Petkov, ${ }^{14}$ J. G. Bian, ${ }^{15}$ G. M. Chen, ${ }^{15}$ H. S. Chen, ${ }^{15}$ C. H. Jiang, ${ }^{15}$ D. Liang, ${ }^{15}$ S. Liang, ${ }^{15}$ X. Meng, ${ }^{15}$ J. Tao, ${ }^{15}$ J. Wang,,${ }^{15}$ J. Wang, ${ }^{15}$ X. Wang, ${ }^{15}$ Z. Wang, ${ }^{15}$ H. Xiao, ${ }^{15}$ M. Xu, ${ }^{15}$ J. Zang, ${ }^{15}$ Z. Zhang, ${ }^{15}$ Y. Ban, ${ }^{16}$ S. Guo, ${ }^{16}$ Y. Guo, ${ }^{16}$ W. Li, ${ }^{16}$ Y. Mao, ${ }^{16}$ S. J. Qian, ${ }^{16}$ H. Teng, ${ }^{16}$

B. Zhu, ${ }^{16}$ W. Zou, ${ }^{16}$ A. Cabrera, ${ }^{17}$ B. Gomez Moreno, ${ }^{17}$ A. A. Ocampo Rios, ${ }^{17}$ A. F. Osorio Oliveros, ${ }^{17}$

J. C. Sanabria, ${ }^{17}$ N. Godinovic, ${ }^{18}$ D. Lelas, ${ }^{18}$ K. Lelas, ${ }^{18}$ R. Plestina,,${ }^{18}$ e D. Polic,${ }^{18}$ I. Puljak, ${ }^{18}$ Z. Antunovic, ${ }^{19}$ M. Dzelalija, ${ }^{19}$ M. Kovac, ${ }^{19}$ V. Brigljevic, ${ }^{20}$ S. Duric,${ }^{20}$ K. Kadija,${ }^{20}$ J. Luetic,${ }^{20}$ S. Morovic, ${ }^{20}$ A. Attikis, ${ }^{21}$ M. Galanti, ${ }^{21}$ J. Mousa,${ }^{21}$ C. Nicolaou ${ }^{21}$ F. Ptochos, ${ }^{21}$ P. A. Razis,${ }^{21}$ M. Finger,${ }^{22}$ M. Finger, Jr., ${ }^{22}$ Y. Assran,${ }^{23, f}$ A. Ellithi Kamel, ${ }^{23, g}$ S. Khalil, ${ }^{23, h}$ M. A. Mahmoud, ${ }^{23, i}$ A. Radi, ${ }^{23, j}$ A. Hektor,${ }^{24}$ M. Kadastik, ${ }^{24}$ M. Müntel, ${ }^{24}$ M. Raidal, ${ }^{24}$ L. Rebane,${ }^{24}$ A. Tiko, ${ }^{24}$ V. Azzolini, ${ }^{25}$ P. Eerola,${ }^{25}$ G. Fedi, ${ }^{25}$ M. Voutilainen, ${ }^{25}$ S. Czellar ${ }^{26}$ J. Härkönen, ${ }^{26}$ A. Heikkinen, ${ }^{26}$ V. Karimäki, ${ }^{26}$ R. Kinnunen, ${ }^{26}$ M. J. Kortelainen, ${ }^{26}$ T. Lampén, ${ }^{26}$ K. Lassila-Perini, ${ }^{26}$ S. Lehti, ${ }^{26}$ T. Lindén, ${ }^{26}$ P. Luukka, ${ }^{26}$ T. Mäenpää, ${ }^{26}$ E. Tuominen, ${ }^{26}$ J. Tuominiemi, ${ }^{26}$ E. Tuovinen, ${ }^{26}$ D. Ungaro, ${ }^{26}$ L. Wendland, ${ }^{26}$ K. Banzuzi, ${ }^{27}$ A. Karjalainen, ${ }^{27}$ A. Korpela, ${ }^{27}$ T. Tuuva, ${ }^{27}$ D. Sillou, ${ }^{28}$ M. Besancon, ${ }^{29}$ S. Choudhury, ${ }^{29}$ M. Dejardin, ${ }^{29}$ D. Denegri, ${ }^{29}$ B. Fabbro, ${ }^{29}$ J. L. Faure, ${ }^{29}$ F. Ferri, ${ }^{29}$ S. Ganjour, ${ }^{29}$ A. Givernaud, ${ }^{29}$ P. Gras, ${ }^{29}$ G. Hamel de Monchenault, ${ }^{29}$ P. Jarry, ${ }^{29}$ E. Locci, ${ }^{29}$ J. Malcles,${ }^{29}$ M. Marionneau, ${ }^{29}$ L. Millischer, ${ }^{29}$ J. Rander, ${ }^{29}$ A. Rosowsky, ${ }^{29}$ I. Shreyber, ${ }^{29}$ M. Titov, ${ }^{29}$ S. Baffioni, ${ }^{30}$ F. Beaudette, ${ }^{30}$ L. Benhabib, ${ }^{30}$ L. Bianchini, ${ }^{30}$ M. Bluj,${ }^{30, k}$ C. Broutin, ${ }^{30}$ P. Busson, ${ }^{30}$ C. Charlot,${ }^{30}$ T. Dahms,${ }^{30}$ L. Dobrzynski,${ }^{30}$ S. Elgammal, ${ }^{30}$

R. Granier de Cassagnac, ${ }^{30}$ M. Haguenauer, ${ }^{30}$ P. Miné, ${ }^{30}$ C. Mironov ${ }^{30}$ C. Ochando,${ }^{30}$ P. Paganini, ${ }^{30}$ D. Sabes,${ }^{30}$ R. Salerno,${ }^{30}$ Y. Sirois,${ }^{30}$ C. Thiebaux,${ }^{30}$ C. Veelken, ${ }^{30}$ A. Zabi,${ }^{30}$ J.-L. Agram,${ }^{31,1}$ J. Andrea, ${ }^{31}$ D. Bloch, ${ }^{31}$ D. Bodin, ${ }^{31}$ J.-M. Brom, ${ }^{31}$ M. Cardaci, ${ }^{31}$ E. C. Chabert ${ }^{31}$ C. Collard,${ }^{31}$ E. Conte, ${ }^{31,1}$ F. Drouhin, ${ }^{31,1}$ C. Ferro, ${ }^{31}$ J.-C. Fontaine, ${ }^{31,1}$ D. Gelé, ${ }^{31}$ U. Goerlach, ${ }^{31}$ S. Greder, ${ }^{31}$ P. Juillot,${ }^{31}$ M. Karim, ${ }^{31,1}$ A.-C. Le Bihan, ${ }^{31}$ Y. Mikami, ${ }^{31}$ P. Van Hove,${ }^{31}$ F. Fassi, ${ }^{32}$ D. Mercier,,${ }^{32}$ C. Baty, ${ }^{33}$ S. Beauceron, ${ }^{33}$ N. Beaupere, ${ }^{33}$ M. Bedjidian, ${ }^{33}$ O. Bondu, ${ }^{33}$ G. Boudoul, ${ }^{33}$ D. Boumediene, ${ }^{33}$ H. Brun, ${ }^{33}$ J. Chasserat,${ }^{33}$ R. Chierici, ${ }^{33}$ D. Contardo,${ }^{33}$ P. Depasse,${ }^{33}$ H. El Mamouni, ${ }^{33}$ J. Fay, ${ }^{33}$ S. Gascon, ${ }^{33}$ B. Ille, ${ }^{33}$ T. Kurca ${ }^{33}$ T. Le Grand,${ }^{33}$ M. Lethuillier, ${ }^{33}$ L. Mirabito, ${ }^{33}$ S. Perries, ${ }^{33}$ V. Sordini, ${ }^{33}$ S. Tosi ${ }^{33}$ Y. Tschudi, ${ }^{33}$ P. Verdier, ${ }^{33}$ S. Viret, ${ }^{33}$ D. Lomidze, ${ }^{34}$ G. Anagnostou, ${ }^{35}$ S. Beranek, ${ }^{35}$ M. Edelhoff, ${ }^{35}$ L. Feld, ${ }^{35}$ N. Heracleous, ${ }^{35}$ O. Hindrichs, ${ }^{35}$ R. Jussen, ${ }^{35}$ K. Klein, ${ }^{35}$ J. Merz, ${ }^{35}$ N. Mohr, ${ }^{35}$ A. Ostapchuk, ${ }^{35}$ A. Perieanu, ${ }^{35}$ F. Raupach,${ }^{35}$ J. Sammet, ${ }^{35}$ S. Schael, ${ }^{35}$ D. Sprenger, ${ }^{35}$ H. Weber, ${ }^{35}$ M. Weber, ${ }^{35}$ B. Wittmer, ${ }^{35}$ V. Zhukov, ${ }^{35, m}$ M. Ata, ${ }^{36}$ E. Dietz-Laursonn, ${ }^{36}$ M. Erdmann, ${ }^{36}$ T. Hebbeker, ${ }^{36}$ C. Heidemann, ${ }^{36}$ A. Hinzmann, ${ }^{36}$ K. Hoepfner ${ }^{36}$ T. Klimkovich, ${ }^{36}$ D. Klingebiel, ${ }^{36}$ P. Kreuzer, ${ }^{36}$ D. Lanske, ${ }^{36, a}$ J. Lingemann, ${ }^{36}$ C. Magass, ${ }^{36}$ M. Merschmeyer,${ }^{36}$ A. Meyer, ${ }^{36}$ P. Papacz, ${ }^{36}$ H. Pieta, ${ }^{36}$ H. Reithler, ${ }^{36}$ S. A. Schmitz, ${ }^{36}$ L. Sonnenschein,${ }^{36}$ J. Steggemann, ${ }^{36}$ D. Teyssier, ${ }^{36}$ M. Bontenackels, ${ }^{37}$ V. Cherepanov,${ }^{37}$ M. Davids, ${ }^{37}$ G. Flügge, ${ }^{37}$ H. Geenen, ${ }^{37}$ M. Giffels, ${ }^{37}$ W. Haj Ahmad,${ }^{37}$ F. Hoehle, ${ }^{37}$ B. Kargoll, ${ }^{37}$ T. Kress,${ }^{37}$ Y. Kuessel,${ }^{37}$ A. Linn, ${ }^{37}$ A. Nowack, ${ }^{37}$ L. Perchalla, ${ }^{37}$ O. Pooth,${ }^{37}$ J. Rennefeld, ${ }^{37}$ P. Sauerland, ${ }^{37}$ A. Stahl,${ }^{37}$ D. Tornier, ${ }^{37}$ M. H. Zoeller, ${ }^{37}$ M. Aldaya Martin, ${ }^{38}$ W. Behrenhoff,${ }^{38}$ U. Behrens, ${ }^{38}$ M. Bergholz,${ }^{38, n}$ A. Bethani, ${ }^{38}$ K. Borras,${ }^{38}$ A. Cakir, ${ }^{38}$ A. Campbell, ${ }^{38}$ E. Castro, ${ }^{38}$ D. Dammann, ${ }^{38}$ G. Eckerlin, ${ }^{38}$ D. Eckstein, ${ }^{38}$ A. Flossdorf, ${ }^{38}$ G. Flucke, ${ }^{38}$ A. Geiser,${ }^{38}$ J. Hauk, ${ }^{38}$ H. Jung, ${ }^{38, b}$ M. Kasemann, ${ }^{38}$ P. Katsas, ${ }^{38}$ C. Kleinwort, ${ }^{38}$ H. Kluge, ${ }^{38}$ A. Knutsson, ${ }^{38}$ M. Krämer, ${ }^{38}$

D. Krücker, ${ }^{38}$ E. Kuznetsova, ${ }^{38}$ W. Lange, ${ }^{38}$ W. Lohmann, ${ }^{38, n}$ B. Lutz, ${ }^{38}$ R. Mankel, ${ }^{38}$ M. Marienfeld, ${ }^{38}$ I.-A. Melzer-Pellmann, ${ }^{38}$ A. B. Meyer, ${ }^{38}$ J. Mnich, ${ }^{38}$ A. Mussgiller, ${ }^{38}$ J. Olzem, ${ }^{38}$ A. Petrukhin, ${ }^{38}$ D. Pitzl, ${ }^{38}$ A. Raspereza, ${ }^{38}$ M. Rosin, ${ }^{38}$ R. Schmidt, ${ }^{38, n}$ T. Schoerner-Sadenius, ${ }^{38}$ N. Sen,${ }^{38}$ A. Spiridonov, ${ }^{38}$ M. Stein, ${ }^{38}$ J. Tomaszewska, ${ }^{38} \mathrm{R}$. Walsh, ${ }^{38} \mathrm{C}$. Wissing, ${ }^{38} \mathrm{C}$. Autermann, ${ }^{39} \mathrm{~V}$. Blobel, ${ }^{39} \mathrm{~S}$. Bobrovskyi, ${ }^{39} \mathrm{~J}$. Draeger ${ }^{39}$ H. Enderle,${ }^{39}$ U. Gebbert, ${ }^{39}$ M. Görner, ${ }^{39}$ T. Hermanns, ${ }^{39}$ K. Kaschube, ${ }^{39}$ G. Kaussen, ${ }^{39}$ H. Kirschenmann, ${ }^{39}$ R. Klanner, ${ }^{39}$ J. Lange, ${ }^{39}$ B. Mura, ${ }^{39}$ S. Naumann-Emme, ${ }^{39}$ F. Nowak,${ }^{39}$ N. Pietsch, ${ }^{39}$ C. Sander, ${ }^{39}$ H. Schettler, ${ }^{39}$ P. Schleper, ${ }^{39}$ E. Schlieckau, ${ }^{39}$ M. Schröder ${ }^{39}$ T. Schum, ${ }^{39}$ H. Stadie, ${ }^{39}$ G. Steinbrück, ${ }^{39}$ J. Thomsen, ${ }^{39}$ C. Barth, ${ }^{40}$ J. Bauer, ${ }^{40}$ J. Berger, ${ }^{40}$ V. Buege, ${ }^{40}$ T. Chwalek, ${ }^{40}$ W. De Boer, ${ }^{40}$ A. Dierlamm,${ }^{40}$ G. Dirkes, ${ }^{40}$ M. Feindt,${ }^{40}$ J. Gruschke, ${ }^{40}$ M. Guthoff, ${ }^{40, b}$ C. Hackstein,${ }^{40}$ F. Hartmann, ${ }^{40}$ M. Heinrich,${ }^{40}$ H. Held, ${ }^{40}$ K. H. Hoffmann, ${ }^{40}$ S. Honc, ${ }^{40}$ I. Katkov, ${ }^{40, m}$ J. R. Komaragiri, ${ }^{40}$ T. Kuhr,${ }^{40}$ D. Martschei, ${ }^{40}$ S. Mueller,${ }^{40}$ Th. Müller, ${ }^{40}$ M. Niegel, ${ }^{40}$ O. Oberst, ${ }^{40}$ A. Oehler, ${ }^{40}$ J. Ott, ${ }^{40}$ T. Peiffer, ${ }^{40}$ G. Quast,${ }^{40}$ K. Rabbertz, ${ }^{40}$ F. Ratnikov,${ }^{40}$ N. Ratnikova, ${ }^{40}$ M. Renz, ${ }^{40}$ S. Röcker, ${ }^{40}$ C. Saout, ${ }^{40}$ A. Scheurer, ${ }^{40}$ P. Schieferdecker, ${ }^{40}$ F.-P. Schilling, ${ }^{40}$ M. Schmanau, ${ }^{40}$ G. Schott,${ }^{40}$ H. J. Simonis, ${ }^{40}$ F. M. Stober, ${ }^{40}$ D. Troendle, ${ }^{40}$ J. Wagner-Kuhr, ${ }^{40}$ T. Weiler, ${ }^{40}$ M. Zeise, ${ }^{40}$ E. B. Ziebarth, ${ }^{40}$ 
G. Daskalakis, ${ }^{41}$ T. Geralis, ${ }^{41}$ S. Kesisoglou, ${ }^{41}$ A. Kyriakis, ${ }^{41}$ D. Loukas, ${ }^{41}$ I. Manolakos, ${ }^{41}$ A. Markou, ${ }^{41}$ C. Markou, ${ }^{41}$ C. Mavrommatis, ${ }^{41}$ E. Ntomari, ${ }^{41}$ E. Petrakou, ${ }^{41}$ L. Gouskos, ${ }^{42}$ T. J. Mertzimekis,${ }^{42}$ A. Panagiotou, ${ }^{42}$ N. Saoulidou, ${ }^{42}$ E. Stiliaris, ${ }^{42}$ I. Evangelou ${ }^{43}$ C. Foudas, ${ }^{43, b}$ P. Kokkas, ${ }^{43}$ N. Manthos, ${ }^{43}$ I. Papadopoulos,${ }^{43}$ V. Patras ${ }^{43}$ F. A. Triantis, ${ }^{43}$ A. Aranyi,${ }^{44}$ G. Bencze, ${ }^{44}$ L. Boldizsar, ${ }^{44}$ C. Hajdu,${ }^{44, b}$ P. Hidas, ${ }^{44}$ D. Horvath, ${ }^{44, o}$ A. Kapusi, ${ }^{44}$ K. Krajczar, ${ }^{4, p}$ F. Sikler, ${ }^{4, b}$ G. I. Veres, ${ }^{44, p}$ G. Vesztergombi, ${ }^{44, p}$ N. Beni, ${ }^{45}$ J. Molnar, ${ }^{45}$ J. Palinkas, ${ }^{45}$ Z. Szillasi, ${ }^{45}$ V. Veszpremi, ${ }^{45}$ J. Karancsi ${ }^{46}$ P. Raics, ${ }^{46}$ Z. L. Trocsanyi, ${ }^{46}$ B. Ujvari ${ }^{46}$ S. B. Beri ${ }^{47}$ V. Bhatnagar, ${ }^{47}$ N. Dhingra, ${ }^{47}$ R. Gupta, ${ }^{47}$ M. Jindal,${ }^{47}$ M. Kaur, ${ }^{47}$ J. M. Kohli, ${ }^{47}$ M. Z. Mehta, ${ }^{47}$ N. Nishu,${ }^{47}$ L. K. Saini,${ }^{47}$ A. Sharma, ${ }^{47}$ A. P. Singh,${ }^{47}$ J. Singh, ${ }^{47}$ S. P. Singh,${ }^{47}$ S. Ahuja, ${ }^{48}$ B. C. Choudhary, ${ }^{48}$ P. Gupta, ${ }^{48}$ A. Kumar, ${ }^{48}$ A. Kumar, ${ }^{48}$ S. Malhotra, ${ }^{48}$ M. Naimuddin, ${ }^{48}$ K. Ranjan, ${ }^{48}$ R. K. Shivpuri, ${ }^{48}$ S. Banerjee ${ }^{49}$ S. Bhattacharya,${ }^{49}$ S. Dutta, ${ }^{49}$ B. Gomber ${ }^{49}$ S. Jain, ${ }^{49}$ S. Jain, ${ }^{49}$ R. Khurana, ${ }^{49}$ S. Sarkar, ${ }^{49}$ R. K. Choudhury, ${ }^{50}$ D. Dutta, ${ }^{50}$ S. Kailas,${ }^{50}$ V. Kumar, ${ }^{50}$ P. Mehta, ${ }^{50}$ A. K. Mohanty, ${ }^{50, \mathrm{~b}}$ L. M. Pant ${ }^{50}$ P. Shukla, ${ }^{50}$ T. Aziz,${ }^{51}$ M. Guchait,${ }^{51, \mathrm{q}}$ A. Gurtu, ${ }^{51}$ M. Maity ${ }^{51, \mathrm{r}}$ D. Majumder, ${ }^{51}$ G. Majumder ${ }^{51}$ K. Mazumdar, ${ }^{51}$ G. B. Mohanty,${ }^{51}$ B. Parida, ${ }^{51}$ A. Saha,${ }^{51}$ K. Sudhakar, ${ }^{51}$ N. Wickramage, ${ }^{51}$ S. Banerjee, ${ }^{52}$ S. Dugad,${ }^{52}$ N. K. Mondal,${ }^{52}$ H. Arfaei,${ }^{53}$ H. Bakhshiansohi,${ }^{53, s}$ S. M. Etesami, ${ }^{53, \mathrm{t}}$ A. Fahim, ${ }^{53, \mathrm{~s}}$ M. Hashemi, ${ }^{53}$ H. Hesari, ${ }^{53}$ A. Jafari, ${ }^{53, \mathrm{~s}}$ M. Khakzad, ${ }^{53}$ A. Mohammadi, ${ }^{53, \mathrm{u}}$ M. Mohammadi Najafabadi, ${ }^{53}$ S. Paktinat Mehdiabadi, ${ }^{53}$ B. Safarzadeh, ${ }^{53}$ M. Zeinali, ${ }^{53, t}$ M. Abbrescia,,${ }^{54,54 b}$ L. Barbone, ${ }^{54 a, 54 b}$ C. Calabria, ${ }^{54 a, 54 b}$ A. Colaleo, ${ }^{54 a}$ D. Creanza, ${ }^{54 a, 54 c}$ N. De Filippis, ${ }^{54 a, 54 c, b}$ M. De Palma, ${ }^{54 a, 54 b}$ L. Fiore ${ }^{54 \mathrm{a}}$ G. Iaselli, ${ }^{54 a, 54 \mathrm{c}}$ L. Lusito, ${ }^{54 \mathrm{a}, 54 \mathrm{~b}}$ G. Maggi, ${ }^{54,54 \mathrm{c}}$ M. Maggi, ${ }^{54 \mathrm{a}}$ N. Manna, ${ }^{54 a, 54 \mathrm{~b}}$ B. Marangelli, ${ }^{54 a, 54 \mathrm{~b}}$ S. My ${ }^{54 a, 54 c}$ S. Nuzzo,${ }^{54 a, 54 b}$ N. Pacifico,${ }^{54 a, 54 b}$ G. A. Pierro,${ }^{54 a}$ A. Pompili,${ }^{54,54 b}$ G. Pugliese,${ }^{54,54 c}$ F. Romano, ${ }^{54 a, 54 c}$ G. Roselli, ${ }^{54 a, 54 b}$ G. Selvaggi,${ }^{54 a, 54 b}$ L. Silvestris, ${ }^{54 a}$ R. Trentadue,${ }^{54 a}$ S. Tupputi, ${ }^{54 a, 54 b}$ G. Zito, ${ }^{54 a}$ G. Abbiendi, ${ }^{55 a}$ A. C. Benvenuti, ${ }^{55 a}$ D. Bonacorsi,${ }^{55 a}$ S. Braibant-Giacomelli, ${ }^{55 a, 55 b}$ L. Brigliadori, ${ }^{55 a}$ P. Capiluppi, ${ }^{55 a, 55 b}$ A. Castro, ${ }^{55 a, 55 b}$ F. R. Cavallo, ${ }^{55 a}$ M. Cuffiani, ${ }^{55 a, 55 b}$ G. M. Dallavalle, ${ }^{55 a}$ F. Fabbri, ${ }^{55 a}$ A. Fanfani, ${ }^{55 a, 55 b}$ D. Fasanella ${ }^{55 a, b}$ P. Giacomelli, ${ }^{55 a}$ M. Giunta, ${ }^{55 a}$ C. Grandi, ${ }^{55 a}$ S. Marcellini, ${ }^{55 a}$ G. Masetti, ${ }^{55 b}$ M. Meneghelli, ${ }^{55 a, 55 b}$ A. Montanari, ${ }^{55 a}$ F. L. Navarria,${ }^{55 a, 55 b}$ F. Odorici, ${ }^{55 a}$ A. Perrotta, ${ }^{55 a}$ F. Primavera ${ }^{55 a}$ A. M. Rossi, ${ }^{55 a, 55 b}$ T. Rovelli, ${ }^{55 a, 55 b}$ G. Siroli, ${ }^{55 a, 55 b}$ R. Travaglini, ${ }^{55 a, 55 b}$ S. Albergo, ${ }^{56 a, 56 b}$ G. Cappello, ${ }^{56 a, 56 b}$ M. Chiorboli, ${ }^{56 a, 56 b}$ S. Costa, ${ }^{56 a, 56 b}$ R. Potenza, ${ }^{56 a, 56 b}$ A. Tricomi, ${ }^{56 a, 56 b}$ C. Tuve,${ }^{56 a, 56 b}$ G. Barbagli, ${ }^{57 a}$ V. Ciulli, ${ }^{57 a, 57 b}$ C. Civinini, ${ }^{57 a}$ R. D’Alessandro, ${ }^{57 a, 57 b}$ E. Focardi, ${ }^{57 a, 57 b}$ S. Frosali, ${ }^{57 a, 57 b}$ E. Gallo, ${ }^{57 a}$ S. Gonzi, ${ }^{57 a, 57 b}$ M. Meschini, ${ }^{57 \mathrm{a}}$ S. Paoletti, ${ }^{57 \mathrm{a}}$ G. Sguazzoni, ${ }^{57 \mathrm{a}}$ A. Tropiano, ${ }^{57 \mathrm{a}, \mathrm{b}}$ L. Benussi, ${ }^{58}$ S. Bianco, ${ }^{58} \mathrm{~S}$. Colafranceschi, ${ }^{58, v}$ F. Fabbri, ${ }^{58}$ D. Piccolo,${ }^{58}$ P. Fabbricatore,${ }^{59}$ R. Musenich, ${ }^{59}$ A. Benaglia,${ }^{60,60 b, b}$ F. De Guio, ${ }^{60 a, 60 b}$

L. Di Matteo, ${ }^{60 a, 60 b}$ S. Gennai, ${ }^{60 a, b}$ A. Ghezzi, ${ }^{60 a, 60 b}$ S. Malvezzi, ${ }^{60 a}$ A. Martelli, ${ }^{60 a, 60 b}$ A. Massironi, ${ }^{60 a, 60 b, b}$ D. Menasce, ${ }^{60 \mathrm{a}}$ L. Moroni, ${ }^{60 \mathrm{a}}$ M. Paganoni $,{ }^{60 a}, 60 \mathrm{~b}$ D. Pedrini,${ }^{60 \mathrm{a}}$ S. Ragazzi, ${ }^{60 a, 60 \mathrm{~b}}$ N. Redaelli, ${ }^{60 \mathrm{a}}$ S. Sala, ${ }^{60 \mathrm{a}}$ T. Tabarelli de Fatis, ${ }^{60 a, 60 b}$ S. Buontempo, ${ }^{61 \mathrm{a}}$ C. A. Carrillo Montoya ${ }^{61 \mathrm{a}, \mathrm{b}}$ N. Cavallo, ${ }^{61 \mathrm{a}, \mathrm{w}}$ A. De Cosa, ${ }^{61 \mathrm{a}, 61 \mathrm{~b}}$ O. Dogangun ${ }^{61 \mathrm{a}, 61 \mathrm{~b}}$ F. Fabozzi, ${ }^{61 \mathrm{a}, \mathrm{w}}$ A. O. M. Iorio, ${ }^{61 \mathrm{a}, \mathrm{b}}$ L. Lista ${ }^{61 \mathrm{a}}$ M. Merola, ${ }^{61 \mathrm{a}, 61 \mathrm{~b}}$ P. Paolucci, ${ }^{61 \mathrm{a}}$ P. Azzi, ${ }^{62 \mathrm{a}}$ N. Bacchetta, ${ }^{62 a, b}$ P. Bellan, ${ }^{62 a, 62 b}$ D. Bisello, ${ }^{62 a, 62 b}$ A. Branca, ${ }^{62 a}$ R. Carlin, ${ }^{62 a, 62 b}$ P. Checchia, ${ }^{62 a}$ T. Dorigo, ${ }^{62 a}$ U. Dosselli, ${ }^{62 a}$ F. Fanzago, ${ }^{62 a}$ F. Gasparini, ${ }^{62 a, 62 b}$ U. Gasparini, ${ }^{62 a, 62 b}$ A. Gozzelino, ${ }^{62 a}$ S. Lacaprara, ${ }^{62 a, x}$ I. Lazzizzera, ${ }^{62 a, 62 \mathrm{c}}$ M. Margoni, ${ }^{62 a, 62 b}$ M. Mazzucato, ${ }^{62 \mathrm{a}}$ A. T. Meneguzzo,${ }^{62 a, 62 \mathrm{~b}}$ M. Nespolo, ${ }^{62 \mathrm{a}, \mathrm{b}}$ L. Perrozzi, ${ }^{62 \mathrm{a}}$

N. Pozzobon, ${ }^{62 a, 62 b}$ P. Ronchese, ${ }^{62 a, 62 b}$ F. Simonetto, ${ }^{62 a, 62 b}$ E. Torassa, ${ }^{62 a}$ M. Tosi, ${ }^{62 a, 62 b, b}$ S. Vanini, ${ }^{62 a, 62 b}$

P. Zotto, ${ }^{62 a, 62 b}$ G. Zumerle, ${ }^{62 a, 62 b}$ P. Baesso, ${ }^{63 a, 63 b}$ U. Berzano, ${ }^{63 a}$ S. P. Ratti, ${ }^{63 a, 63 b}$ C. Riccardi, ${ }^{63 a, 63 b}$ P. Torre, ${ }^{63 a, 63 b}$ P. Vitulo, ${ }^{63 a, 63 b}$ C. Viviani, ${ }^{63 a, 63 b}$ M. Biasini, ${ }^{64 a, 64 b}$ G. M. Bilei, ${ }^{64 a}$ B. Caponeri, ${ }^{64 a, 64 b}$ L. Fanò, ${ }^{64 a, 64 b}$

P. Lariccia, ${ }^{64 a, 64 b}$ A. Lucaroni ${ }^{64 a, 64 b, b}$ G. Mantovani,${ }^{64 a, 64 b}$ M. Menichelli, ${ }^{64 a}$ A. Nappi ${ }^{64 a, 64 b}$ F. Romeo, ${ }^{64 a, 64 b}$ A. Santocchia, ${ }^{64 a, 64 b}$ S. Taroni, ${ }^{64 a, 64 b, b}$ M. Valdata, ${ }^{64 a, 64 b}$ P. Azzurri, ${ }^{65 a, 65 c}$ G. Bagliesi, ${ }^{65 a}$ J. Bernardini, ${ }^{65 a, 65 b}$ T. Boccali, ${ }^{65 a}$ G. Broccolo, ${ }^{65 a, 65 c}$ R. Castaldi, ${ }^{65 a}$ R. T. D'Agnolo,${ }^{65 a, 65 c}$ R. Dell'Orso, ${ }^{65 a}$ F. Fiori ${ }^{65 a, 65 b}$ L. Foà, ${ }^{65 a, 65 c}$

A. Giassi ${ }^{65 a}$ A. Kraan ${ }^{65 a}$ F. Ligabue,${ }^{65 a, 65 c}$ T. Lomtadze, ${ }^{65 a}$ L. Martini, ${ }^{65 a, y}$ A. Messineo ${ }^{65 a, 65 b}$ F. Palla, ${ }^{65 a}$ F. Palmonari, ${ }^{65 a}$ G. Segneri, ${ }^{65 a}$ A. T. Serban, ${ }^{65 a}$ P. Spagnolo, ${ }^{65 a}$ R. Tenchini, ${ }^{65 a}$ G. Tonelli, ${ }^{65 a, 65 b, b}$ A. Venturi, ${ }^{65 a, b}$ P. G. Verdini, ${ }^{65 a}$ L. Barone, ${ }^{66 a, 66 b}$ F. Cavallari, ${ }^{66 a}$ D. Del Re, ${ }^{66 a, 66 b, b}$ E. Di Marco, ${ }^{66 a, 66 b}$ M. Diemoz, ${ }^{66 a}$

D. Franci, ${ }^{66 a, 66 b}$ M. Grassi, ${ }^{66 a, b}$ E. Longo, ${ }^{66 a, 66 b}$ P. Meridiani, ${ }^{66 a}$ S. Nourbakhsh, ${ }^{66 a}$ G. Organtini, ${ }^{66 a, 66 b}$ F. Pandolfi, ${ }^{66 a, 66 b}$ R. Paramatti, ${ }^{66 a}$ S. Rahatlou, ${ }^{66 a, 66 b}$ M. Sigamani, ${ }^{66 a}$ N. Amapane, ${ }^{67 a, 67 b}$ R. Arcidiacono, ${ }^{67 a, 67 c}$ S. Argiro, ${ }^{67 a, 67 b}$ M. Arneodo, ${ }^{67 a, 67 \mathrm{c}}$ C. Biino, ${ }^{67 \mathrm{a}}$ C. Botta, ${ }^{67 a, 67 b}$ N. Cartiglia ${ }^{67 \mathrm{a}}$ R. Castello, ${ }^{67 \mathrm{a}, 67 \mathrm{~b}} \mathrm{M}$. Costa, ${ }^{67 \mathrm{a}, 67 \mathrm{~b}}$ N. Demaria, ${ }^{67 \mathrm{a}}$ A. Graziano, ${ }^{67 \mathrm{a}, 67 \mathrm{~b}}$ C. Mariotti, ${ }^{67 \mathrm{a}}$ S. Maselli, ${ }^{67 \mathrm{a}}$ E. Migliore, ${ }^{67 \mathrm{a}, 67 \mathrm{~b}}$ V. Monaco, ${ }^{67 \mathrm{a}, 67 \mathrm{~b}}$ M. Musich,${ }^{67 \mathrm{a}}$ M. M. Obertino, ${ }^{67 a, 67 \mathrm{c}}$ N. Pastrone, ${ }^{67 \mathrm{a}}$ M. Pelliccioni, ${ }^{67 \mathrm{a}, 67 \mathrm{~b}}$ A. Potenza,${ }^{67 a, 67 b}$ A. Romero, ${ }^{67 a, 67 b}$ M. Ruspa ${ }^{67 a, 67 \mathrm{c}}$ R. Sacchi, ${ }^{67 a, 67 b}$ V. Sola, ${ }^{67 a, 67 b}$ A. Solano, ${ }^{67 a, 67 b}$ A. Staiano, ${ }^{67 a}$ A. Vilela Pereira, ${ }^{67 a}$ S. Belforte, ${ }^{68 a}$ F. Cossutti, ${ }^{68 a}$ G. Della Ricca, ${ }^{68 a, 68 b}$ B. Gobbo, ${ }^{68 a}$ M. Marone,${ }^{68 a, 68 b}$ D. Montanino, ${ }^{68 a, 68 b}$ A. Penzo, ${ }^{68 a}$ S. G. Heo, ${ }^{69}$ S. K. Nam, ${ }^{69}$ 
S. Chang, ${ }^{70}$ J. Chung,${ }^{70}$ D. H. Kim,${ }^{70}$ G. N. Kim, ${ }^{70}$ J. E. Kim,${ }^{70}$ D. J. Kong, ${ }^{70}$ H. Park, ${ }^{70}$ S. R. Ro, ${ }^{70}$ D. C. Son, ${ }^{70}$ T. Son, ${ }^{70}$ J. Y. Kim, ${ }^{71}$ Zero J. Kim, ${ }^{71}$ S. Song, ${ }^{71}$ H. Y. Jo, ${ }^{72}$ S. Choi,${ }^{73}$ D. Gyun,${ }^{73}$ B. Hong, ${ }^{73}$ M. Jo ${ }^{73}$ H. Kim,${ }^{73}$

T. J. Kim, ${ }^{73}$ K. S. Lee, ${ }^{73}$ D. H. Moon, ${ }^{73}$ S. K. Park, ${ }^{73}$ E. Seo,${ }^{73}$ K. S. Sim,${ }^{73}$ M. Choi,${ }^{74}$ S. Kang, ${ }^{74}$ H. Kim, ${ }^{74}$ J. H. Kim, ${ }^{74}$ C. Park, ${ }^{74}$ I. C. Park, ${ }^{74}$ S. Park,${ }^{74}$ G. Ryu ${ }^{74}$ Y. Cho, ${ }^{75}$ Y. Choi,${ }^{75}$ Y. K. Choi, ${ }^{75}$ J. Goh,${ }^{75}$ M. S. Kim, ${ }^{75}$ B. Lee, ${ }^{75}$ J. Lee, ${ }^{75}$ S. Lee, ${ }^{75}$ H. Seo,${ }^{75}$ I. Yu ${ }^{75}$ M. J. Bilinskas,${ }^{76}$ I. Grigelionis,${ }^{76}$ M. Janulis, ${ }^{76}$ D. Martisiute, ${ }^{76}$ P. Petrov, ${ }^{76}$ M. Polujanskas, ${ }^{76}$ T. Sabonis, ${ }^{76}$ H. Castilla-Valdez,${ }^{77}$ E. De La Cruz-Burelo, ${ }^{77}$ I. Heredia-de La Cruz, ${ }^{77}$ R. Lopez-Fernandez, ${ }^{77}$ R. Magaña Villalba, ${ }^{77}$ J. Martínez-Ortega, ${ }^{77}$ A. Sánchez-Hernández, ${ }^{77}$

L. M. Villasenor-Cendejas, ${ }^{77}$ S. Carrillo Moreno, ${ }^{78}$ F. Vazquez Valencia, ${ }^{78}$ H. A. Salazar Ibarguen, ${ }^{79}$

E. Casimiro Linares,${ }^{80}$ A. Morelos Pineda, ${ }^{80}$ M. A. Reyes-Santos,${ }^{80}$ D. Krofcheck,${ }^{81}$ J. Tam,${ }^{81}$ P. H. Butler, ${ }^{82}$

R. Doesburg, ${ }^{82}$ H. Silverwood, ${ }^{82}$ M. Ahmad, ${ }^{83}$ I. Ahmed, ${ }^{83}$ M. I. Asghar, ${ }^{83}$ H. R. Hoorani, ${ }^{83}$ S. Khalid, ${ }^{83}$ W. A. Khan, ${ }^{83}$ T. Khurshid, ${ }^{83}$ S. Qazi, ${ }^{83}$ M. A. Shah, ${ }^{83}$ M. Shoaib,${ }^{83}$ G. Brona, ${ }^{84}$ M. Cwiok ${ }^{84}$ W. Dominik ${ }^{84}$ K. Doroba ${ }^{84}$ A. Kalinowski, ${ }^{84}$ M. Konecki, ${ }^{84}$ J. Krolikowski, ${ }^{84}$ T. Frueboes, ${ }^{85}$ R. Gokieli, ${ }^{85}$ M. Górski, ${ }^{85}$ M. Kazana, ${ }^{85}$ K. Nawrocki, ${ }^{85}$ K. Romanowska-Rybinska, ${ }^{85}$ M. Szleper, ${ }^{85}$ G. Wrochna ${ }^{85}$ P. Zalewski, ${ }^{85}$ N. Almeida, ${ }^{86}$ P. Bargassa, ${ }^{86}$ A. David, ${ }^{86}$ P. Faccioli, ${ }^{86}$ P. G. Ferreira Parracho, ${ }^{86}$ M. Gallinaro, ${ }^{86, b}$ P. Musella, ${ }^{86}$ A. Nayak ${ }^{86}$ J. Pela,${ }^{86, b}$ P. Q. Ribeiro, ${ }^{86}$ J. Seixas, ${ }^{86}$ J. Varela, ${ }^{86}$ S. Afanasiev, ${ }^{87}$ I. Belotelov, ${ }^{87}$ P. Bunin, ${ }^{87}$ M. Gavrilenko, ${ }^{87}$ I. Golutvin, ${ }^{87}$ A. Kamenev, ${ }^{87}$ V. Karjavin,${ }^{87}$ G. Kozlov, ${ }^{87}$ A. Lanev, ${ }^{87}$ P. Moisenz, ${ }^{87}$ V. Palichik, ${ }^{87}$ V. Perelygin, ${ }^{87}$ S. Shmatov, ${ }^{87}$ V. Smirnov, ${ }^{87}$ A. Volodko, ${ }^{87}$ A. Zarubin, ${ }^{87}$ V. Golovtsov, ${ }^{88}$ Y. Ivanov, ${ }^{88}$ V. Kim, ${ }^{88}$ P. Levchenko, ${ }^{88}$ V. Murzin, ${ }^{88}$ V. Oreshkin, ${ }^{88}$ I. Smirnov, ${ }^{88}$ V. Sulimov, ${ }^{88}$ L. Uvarov, ${ }^{88}$ S. Vavilov, ${ }^{88}$ A. Vorobyev ${ }^{88}$ An. Vorobyev, ${ }^{88}$ Yu. Andreev, ${ }^{89}$ A. Dermenev, ${ }^{89}$ S. Gninenko, ${ }^{89}$ N. Golubev,${ }^{89}$ M. Kirsanov, ${ }^{89}$ N. Krasnikov, ${ }^{89}$

V. Matveev, ${ }^{89}$ A. Pashenkov, ${ }^{89}$ A. Toropin, ${ }^{89}$ S. Troitsky, ${ }^{89}$ V. Epshteyn, ${ }^{90}$ M. Erofeeva, ${ }^{90}$ V. Gavrilov, ${ }^{90}$ V. Kaftanov, ${ }^{90, a}$ M. Kossov, ${ }^{90, b}$ A. Krokhotin, ${ }^{90}$ N. Lychkovskaya, ${ }^{90}$ V. Popov,${ }^{90}$ G. Safronov, ${ }^{90}$ S. Semenov, ${ }^{90}$ V. Stolin, ${ }^{90}$ E. Vlasov, ${ }^{90}$ A. Zhokin, ${ }^{90}$ A. Belyaev,${ }^{91}$ E. Boos, ${ }^{91}$ M. Dubinin,,${ }^{91, d}$ L. Dudko, ${ }^{91}$ A. Ershov, ${ }^{91}$ A. Gribushin, ${ }^{91}$ O. Kodolova, ${ }^{91}$ I. Lokhtin,,${ }^{91}$ A. Markina, ${ }^{91}$ S. Obraztsov, ${ }^{91}$ M. Perfilov, ${ }^{91}$ S. Petrushanko,,${ }^{91}$ L. Sarycheva, ${ }^{91}$ V. Savrin, ${ }^{91}$ A. Snigirev, ${ }^{91}$ V. Andreev, ${ }^{92}$ M. Azarkin, ${ }^{92}$ I. Dremin, ${ }^{92}$ M. Kirakosyan, ${ }^{92}$ A. Leonidov, ${ }^{92}$ G. Mesyats, ${ }^{92}$ S. V. Rusakov, ${ }^{92}$ A. Vinogradov, ${ }^{92}$ I. Azhgirey, ${ }^{93}$ I. Bayshev, ${ }^{93}$ S. Bitioukov, ${ }^{93}$ V. Grishin, ${ }^{93, b}$ V. Kachanov, ${ }^{93}$ D. Konstantinov, ${ }^{93}$ A. Korablev, ${ }^{93}$ V. Krychkine, ${ }^{93}$ V. Petrov, ${ }^{93}$ R. Ryutin, ${ }^{93}$ A. Sobol, ${ }^{93}$ L. Tourtchanovitch, ${ }^{93}$ S. Troshin,${ }^{93}$ N. Tyurin, ${ }^{93}$ A. Uzunian, ${ }^{93}$ A. Volkov, ${ }^{93}$ P. Adzic,${ }^{94, z}$ M. Djordjevic,${ }^{94}$ M. Ekmedzic, ${ }^{94}$ D. Krpic, ${ }^{94, z}$ J. Milosevic,${ }^{94}$ M. Aguilar-Benitez, ${ }^{95}$ J. Alcaraz Maestre, ${ }^{95}$ P. Arce, ${ }^{95}$ C. Battilana, ${ }^{95}$ E. Calvo,${ }^{95}$ M. Cerrada, ${ }^{95}$ M. Chamizo Llatas, ${ }^{95}$ N. Colino, ${ }^{95}$ B. De La Cruz,${ }^{95}$ A. Delgado Peris, ${ }^{95}$ C. Diez Pardos, ${ }^{95}$ D. Domínguez Vázquez ${ }^{95}$ C. Fernandez Bedoya,${ }^{95}$ J. P. Fernández Ramos, ${ }^{95}$ A. Ferrando, ${ }^{95}$ J. Flix, ${ }^{95}$ M. C. Fouz, ${ }^{95}$ P. Garcia-Abia, ${ }^{95}$ O. Gonzalez Lopez,${ }^{95}$ S. Goy Lopez, ${ }^{95}$ J. M. Hernandez, ${ }^{95}$ M. I. Josa, ${ }^{95}$ G. Merino, ${ }^{95}$

J. Puerta Pelayo, ${ }^{95}$ I. Redondo, ${ }^{95}$ L. Romero, ${ }^{95}$ J. Santaolalla, ${ }^{95}$ M. S. Soares,${ }^{95}$ C. Willmott, ${ }^{95}$ C. Albajar, ${ }^{96}$ G. Codispoti, ${ }^{96}$ J. F. de Trocóniz, ${ }^{96}$ J. Cuevas, ${ }^{97}$ J. Fernandez Menendez, ${ }^{97}$ S. Folgueras, ${ }^{97}$ I. Gonzalez Caballero, ${ }^{97}$ L. Lloret Iglesias, ${ }^{97}$ J. M. Vizan Garcia, ${ }^{97}$ J. A. Brochero Cifuentes, ${ }^{98}$ I. J. Cabrillo, ${ }^{98}$ A. Calderon, ${ }^{98}$ S. H. Chuang, ${ }^{98}$

J. Duarte Campderros, ${ }^{98}$ M. Felcini, ${ }^{98, a a}$ M. Fernandez,${ }^{98}$ G. Gomez, ${ }^{98}$ J. Gonzalez Sanchez, ${ }^{98}$ C. Jorda, ${ }^{98}$

P. Lobelle Pardo, ${ }^{98}$ A. Lopez Virto, ${ }^{98}$ J. Marco, ${ }^{98}$ R. Marco,${ }^{98}$ C. Martinez Rivero, ${ }^{98}$ F. Matorras, ${ }^{98}$

F. J. Munoz Sanchez, ${ }^{98}$ J. Piedra Gomez, ${ }^{98, b b}$ T. Rodrigo, ${ }^{98}$ A. Y. Rodríguez-Marrero, ${ }^{98}$ A. Ruiz-Jimeno, ${ }^{98}$

L. Scodellaro, ${ }^{98}$ M. Sobron Sanudo, ${ }^{98}$ I. Vila, ${ }^{98}$ R. Vilar Cortabitarte, ${ }^{98}$ D. Abbaneo,${ }^{99}$ E. Auffray, ${ }^{99}$ G. Auzinger, ${ }^{99}$

P. Baillon, ${ }^{99}$ A. H. Ball, ${ }^{99}$ D. Barney, ${ }^{99}$ A. J. Bell, ${ }^{99, c c}$ D. Benedetti, ${ }^{99}$ C. Bernet, ${ }^{99, e}$ W. Bialas, ${ }^{99}$ P. Bloch,${ }^{99}$

A. Bocci, ${ }^{99}$ S. Bolognesi, ${ }^{99}$ M. Bona, ${ }^{99}$ H. Breuker, ${ }^{99}$ K. Bunkowski, ${ }^{99}$ T. Camporesi, ${ }^{99}$ G. Cerminara,${ }^{99}$

T. Christiansen, ${ }^{99}$ J. A. Coarasa Perez,${ }^{99}$ B. Curé, ${ }^{99}$ D. D'Enterria, ${ }^{99}$ A. De Roeck, ${ }^{99}$ S. Di Guida, ${ }^{99}$

N. Dupont-Sagorin, ${ }^{99}$ A. Elliott-Peisert, ${ }^{99}$ B. Frisch,${ }^{99}$ W. Funk,${ }^{99}$ A. Gaddi, ${ }^{99}$ G. Georgiou, ${ }^{99}$ H. Gerwig,,${ }^{99}$ D. Gigi, ${ }^{99}$ K. Gill, ${ }^{99}$ D. Giordano, ${ }^{99}$ F. Glege, ${ }^{99}$ R. Gomez-Reino Garrido, ${ }^{99}$ M. Gouzevitch, ${ }^{99}$ P. Govoni, ${ }^{99}$ S. Gowdy, ${ }^{99}$ R. Guida, ${ }^{99}$ L. Guiducci, ${ }^{99}$ M. Hansen, ${ }^{99}$ C. Hartl, ${ }^{99}$ J. Harvey, ${ }^{99}$ J. Hegeman, ${ }^{99}$ B. Hegner, ${ }^{99}$ H. F. Hoffmann, ${ }^{99}$ V. Innocente, ${ }^{99}$ P. Janot, ${ }^{99}$ K. Kaadze, ${ }^{99}$ E. Karavakis, ${ }^{99}$ P. Lecoq,${ }^{99}$ P. Lenzi, ${ }^{99}$ C. Lourenço, ${ }^{99}$ T. Mäki, ${ }^{99}$ M. Malberti, ${ }^{99}$ L. Malgeri, ${ }^{99}$ M. Mannelli, ${ }^{99}$ L. Masetti, ${ }^{99}$ A. Maurisset, ${ }^{99}$ G. Mavromanolakis,${ }^{99}$ F. Meijers, ${ }^{99}$ S. Mersi,${ }^{99}$ E. Meschi, ${ }^{99}$ R. Moser,${ }^{99}$ M. U. Mozer, ${ }^{99}$ M. Mulders,${ }^{99}$ E. Nesvold, ${ }^{99}$ M. Nguyen, ${ }^{99}$ T. Orimoto, ${ }^{99}$ L. Orsini,${ }^{99}$ E. Palencia Cortezon, ${ }^{99}$ E. Perez, ${ }^{99}$ A. Petrilli, ${ }^{99}$ A. Pfeiffer, ${ }^{99}$ M. Pierini, ${ }^{99}$ M. Pimiä, ${ }^{99}$ D. Piparo, ${ }^{99}$ G. Polese, ${ }^{99}$ L. Quertenmont, ${ }^{99}$ A. Racz, ${ }^{99}$ W. Reece, ${ }^{99}$ J. Rodrigues Antunes, ${ }^{99}$ G. Rolandi, ${ }^{99, d d}$ T. Rommerskirchen, ${ }^{99}$ C. Rovelli, $,{ }^{99}, \mathrm{ee}$ M. Rovere, ${ }^{99}$ H. Sakulin, ${ }^{99}$ C. Schäfer, ${ }^{99}$ C. Schwick, ${ }^{99}$ I. Segoni, ${ }^{99}$ A. Sharma,${ }^{99}$ P. Siegrist,${ }^{99}$ P. Silva,${ }^{99}$ M. Simon,,${ }^{99}$ P. Sphicas, ${ }^{99, f f}$ D. Spiga, ${ }^{99}$ M. Spiropulu, ${ }^{99, d}$ M. Stoye,${ }^{99}$ 
A. Tsirou, ${ }^{99}$ P. Vichoudis, ${ }^{99}$ H. K. Wöhri, ${ }^{99}$ S. D. Worm, ${ }^{99}$ W. D. Zeuner, ${ }^{99}$ W. Bertl, ${ }^{100}$ K. Deiters,${ }^{100}$ W. Erdmann, ${ }^{100}$ K. Gabathuler, ${ }^{100}$ R. Horisberger, ${ }^{100}$ Q. Ingram, ${ }^{100}$ H. C. Kaestlii, ${ }^{100}$ S. König, ${ }^{100}$ D. Kotlinski, ${ }^{100}$ U. Langenegger, ${ }^{100}$ F. Meier, ${ }^{100}$ D. Renker, ${ }^{100}$ T. Rohe,${ }^{100}$ J. Sibille, ${ }^{100, g g}$ L. Bäni, ${ }^{101}$ P. Bortignon, ${ }^{101}$ L. Caminada, ${ }^{101, h h}$ B. Casal, ${ }^{101}$ N. Chanon, ${ }^{101}$ Z. Chen, ${ }^{101}$ S. Cittolin, ${ }^{101}$ G. Dissertori, ${ }^{101}$ M. Dittmar, ${ }^{101}$ J. Eugster, ${ }^{101}$ K. Freudenreich,,${ }^{101}$ C. Grab, ${ }^{101}$ W. Hintz,${ }^{101}$ P. Lecomte, ${ }^{101}$ W. Lustermann,,${ }^{101}$ C. Marchica,,${ }^{101, \text { hh }}$ P. Martinez Ruiz del Arbol, ${ }^{101}$ P. Milenovic, ${ }^{101, \text { ii }}$ F. Moortgat, ${ }^{101}$ C. Nägeli, ${ }^{101, \text { hh }}$ P. Nef, ${ }^{101}$ F. Nessi-Tedaldi, ${ }^{101}$ L. Pape, ${ }^{101}$ F. Pauss,${ }^{101}$ T. Punz, ${ }^{101}$ A. Rizzi, ${ }^{101}$ F. J. Ronga, ${ }^{101}$ M. Rossini, ${ }^{101}$ L. Sala, ${ }^{101}$ A. K. Sanchez, ${ }^{101}$ M.-C. Sawley, ${ }^{101}$ A. Starodumov,${ }^{101, j j}$ B. Stieger, ${ }^{101}$ M. Takahashi, ${ }^{101}$ L. Tauscher, ${ }^{101, a}$ A. Thea, ${ }^{101}$ K. Theofilatos,${ }^{101}$ D. Treille, ${ }^{101}$ C. Urscheler,${ }^{101}$ R. Wallny, ${ }^{101}$ M. Weber ${ }^{101}$ L. Wehrli,,${ }^{101} \mathrm{~J}$. Weng, ${ }^{101}$ E. Aguilo, ${ }^{102}$ C. Amsler, ${ }^{102}$ V. Chiochia, ${ }^{102}$ S. De Visscher, ${ }^{102}$ C. Favaro, ${ }^{102}$ M. Ivova Rikova, ${ }^{102}$ A. Jaeger, ${ }^{102}$ B. Millan Mejias, ${ }^{102}$ P. Otiougova, ${ }^{102}$ P. Robmann, ${ }^{102}$ A. Schmidt,${ }^{102}$

H. Snoek, ${ }^{102}$ Y. H. Chang, ${ }^{103}$ K. H. Chen, ${ }^{103}$ C. M. Kuo, ${ }^{103}$ S. W. Li,${ }^{103}$ W. Lin, ${ }^{103}$ Z. K. Liu, ${ }^{103}$ Y. J. Lu, ${ }^{103}$ D. Mekterovic, ${ }^{103}$ R. Volpe,${ }^{103}$ S. S. Yu, ${ }^{103}$ P. Bartalini, ${ }^{104}$ P. Chang, ${ }^{104}$ Y. H. Chang, ${ }^{104}$ Y. W. Chang, ${ }^{104}$ Y. Chao ${ }^{104}$ K. F. Chen, ${ }^{104}$ C. Dietz, ${ }^{104}$ U. Grundler, ${ }^{104}$ W.-S. Hou, ${ }^{104}$ Y. Hsiung,,${ }^{104}$ K. Y. Kao, ${ }^{104}$ Y. J. Lei, ${ }^{104}$ R.-S. Lu, ${ }^{104}$ J. G. Shiu, ${ }^{104}$ Y. M. Tzeng, ${ }^{104}$ X. Wan, ${ }^{104}$ M. Wang, ${ }^{104}$ A. Adiguzel, ${ }^{105}$ M. N. Bakirci, ${ }^{105, k k}$ S. Cerci, ${ }^{105,11}$ C. Dozen, ${ }^{105}$ I. Dumanoglu, ${ }^{105}$ E. Eskut, ${ }^{105}$ S. Girgis, ${ }^{105}$ G. Gokbulut, ${ }^{105}$ I. Hos, ${ }^{105}$ E. E. Kangal,${ }^{105}$ A. Kayis Topaksu, ${ }^{105}$ G. Onengut, ${ }^{105}$ K. Ozdemir, ${ }^{105}$ S. Ozturk, ${ }^{105, m m}$ A. Polatoz, ${ }^{105}$ K. Sogut,,${ }^{105, n n}$ D. Sunar Cerci, ${ }^{105,11}$ B. Tali, ${ }^{105,11}$ H. Topakli, ${ }^{105, k k}$ D. Uzun, ${ }^{105}$ L. N. Vergili, ${ }^{105}$ M. Vergili, ${ }^{105}$ I. V. Akin,,${ }^{106}$ T. Aliev, ${ }^{106}$ B. Bilin, ${ }^{106}$ S. Bilmis, ${ }^{106}$ M. Deniz, ${ }^{106}$ H. Gamsizkan, ${ }^{106}$ A. M. Guler, ${ }^{106}$ K. Ocalan, ${ }^{106}$ A. Ozpineci, ${ }^{106}$ M. Serin, ${ }^{106}$ R. Sever, ${ }^{106}$ U.E. Surat, ${ }^{106}$ M. Yalvac, ${ }^{106}$ E. Yildirim,,${ }^{106}$ M. Zeyrek, ${ }^{106}$ M. Deliomeroglu, ${ }^{107}$

E. Gülmez, ${ }^{107}$ B. Isildak, ${ }^{107}$ M. Kaya, ${ }^{107, \text { oo }}$ O. Kaya, ${ }^{107,00}$ M. Özbek, ${ }^{107}$ S. Ozkorucuklu, ${ }^{107, p p}$ N. Sonmez, ${ }^{107, q q}$ L. Levchuk, ${ }^{108}$ F. Bostock, ${ }^{109}$ J. J. Brooke, ${ }^{109}$ T. L. Cheng,,${ }^{109}$ E. Clement,,${ }^{109}$ D. Cussans, ${ }^{109}$ R. Frazier,,${ }^{109}$ J. Goldstein, ${ }^{109}$ M. Grimes, ${ }^{109}$ G. P. Heath,${ }^{109}$ H. F. Heath,${ }^{109}$ L. Kreczko, ${ }^{109}$ S. Metson, ${ }^{109}$ D. M. Newbold,${ }^{109, \text { rr }}$ K. Nirunpong, ${ }^{109}$ A. Poll, ${ }^{109}$ S. Senkin, ${ }^{109}$ V. J. Smith, ${ }^{109}$ L. Basso, ${ }^{110, s s}$ K. W. Bell, ${ }^{110}$ A. Belyaev, ${ }^{110, s s}$ C. Brew, ${ }^{110}$ R. M. Brown, ${ }^{110}$ B. Camanzi, ${ }^{110}$ D. J. A. Cockerill, ${ }^{110}$ J. A. Coughlan, ${ }^{110}$ K. Harder, ${ }^{110}$ S. Harper, ${ }^{110}$ J. Jackson, ${ }^{110}$ B.W. Kennedy, ${ }^{110}$ E. Olaiya, ${ }^{110}$ D. Petyt, ${ }^{110}$ B. C. Radburn-Smith, ${ }^{110}$ C. H. Shepherd-Themistocleous, ${ }^{110}$ I. R. Tomalin, ${ }^{110}$ W. J. Womersley, ${ }^{110}$ R. Bainbridge, ${ }^{111}$ G. Ball, ${ }^{111}$ J. Ballin, ${ }^{111}$ R. Beuselinck, ${ }^{111}$ O. Buchmuller,${ }^{111}$ D. Colling, ${ }^{111}$ N. Cripps, ${ }^{111}$ M. Cutajar,${ }^{111}$ G. Davies,${ }^{111}$ M. Della Negra, ${ }^{111}$ W. Ferguson, ${ }^{111}$ J. Fulcher,${ }^{111}$ D. Futyan, ${ }^{111}$ A. Gilbert, ${ }^{111}$ A. Guneratne Bryer, ${ }^{111}$ G. Hall, ${ }^{111}$ Z. Hatherell, ${ }^{111}$ J. Hays, ${ }^{111}$ G. Iles,${ }^{111}$ M. Jarvis, ${ }^{111}$ G. Karapostoli, ${ }^{111}$ L. Lyons, ${ }^{111}$ A.-M. Magnan, ${ }^{111}$ J. Marrouche, ${ }^{111}$ B. Mathias, ${ }^{111}$ R. Nandi, ${ }^{111}$ J. Nash, ${ }^{111}$ A. Nikitenko, ${ }^{111, j j}$ A. Papageorgiou, ${ }^{111}$ M. Pesaresi, ${ }^{111}$ K. Petridis,${ }^{111}$ M. Pioppi, ${ }^{111, t t}$ D. M. Raymond, ${ }^{111}$ S. Rogerson, ${ }^{111}$ N. Rompotis, ${ }^{111}$ A. Rose, ${ }^{111}$ M. J. Ryan, ${ }^{111}$ C. Seez,${ }^{111}$ P. Sharp,${ }^{111}$ A. Sparrow, ${ }^{111}$ A. Tapper, ${ }^{111}$ S. Tourneur, ${ }^{111} \mathrm{M}$. Vazquez Acosta, ${ }^{111}$ T. Virdee, ${ }^{111} \mathrm{~S}$. Wakefield, ${ }^{111} \mathrm{~N}$. Wardle, ${ }^{111} \mathrm{D}$. Wardrope,${ }^{111} \mathrm{~T}$. Whyntie, ${ }^{111}$ M. Barrett, ${ }^{112}$ M. Chadwick, ${ }^{112}$ J. E. Cole, ${ }^{112}$ P. R. Hobson, ${ }^{112}$ A. Khan, ${ }^{112}$ P. Kyberd, ${ }^{112}$ D. Leslie, ${ }^{112}$ W. Martin, ${ }^{112}$ I. D. Reid,${ }^{112}$ L. Teodorescu, ${ }^{112}$ K. Hatakeyama, ${ }^{113}$ H. Liu, ${ }^{113}$ C. Henderson, ${ }^{114}$ T. Bose, ${ }^{115}$ E. Carrera Jarrin, ${ }^{115}$ C. Fantasia, ${ }^{115}$ A. Heister, ${ }^{115}$ J. St. John, ${ }^{115}$ P. Lawson, ${ }^{115}$ D. Lazic, ${ }^{115}$ J. Rohlf, ${ }^{115}$ D. Sperka, ${ }^{115}$ L. Sulak, ${ }^{115}$ A. Avetisyan, ${ }^{116}$ S. Bhattacharya, ${ }^{116}$ J. P. Chou, ${ }^{116}$ D. Cutts, ${ }^{116}$ A. Ferapontov, ${ }^{116}$ U. Heintz, ${ }^{116}$ S. Jabeen, ${ }^{116}$ G. Kukartsev, ${ }^{116}$ G. Landsberg, ${ }^{116}$ M. Luk, ${ }^{116}$ M. Narain, ${ }^{116}$ D. Nguyen, ${ }^{116}$ M. Segala, ${ }^{116}$ T. Sinthuprasith, ${ }^{116}$ T. Speer, ${ }^{116}$ K. V. Tsang, ${ }^{116}$ R. Breedon, ${ }^{117}$ G. Breto, ${ }^{117}$ M. Calderon De La Barca Sanchez, ${ }^{117}$ S. Chauhan, ${ }^{117}$ M. Chertok, ${ }^{117}$ J. Conway, ${ }^{117}$ R. Conway, ${ }^{117}$ P. T. Cox,${ }^{117}$ J. Dolen, ${ }^{117}$ R. Erbacher, ${ }^{117}$ R. Houtz,${ }^{117}$ W. Ko, ${ }^{117}$ A. Kopecky, ${ }^{117}$ R. Lander, ${ }^{117}$ H. Liu, ${ }^{117}$ O. Mall, ${ }^{117}$ S. Maruyama, ${ }^{117}$ T. Miceli, ${ }^{117}$ M. Nikolic, ${ }^{117}$ D. Pellett, ${ }^{117}$ J. Robles,${ }^{117}$ B. Rutherford, ${ }^{117}$ S. Salur, ${ }^{117}$ M. Searle, ${ }^{117}$ J. Smith, ${ }^{117}$ M. Squires, ${ }^{117}$ M. Tripathi, ${ }^{117}$

R. Vasquez Sierra, ${ }^{117}$ V. Andreev,${ }^{118}$ K. Arisaka, ${ }^{118}$ D. Cline, ${ }^{118}$ R. Cousins, ${ }^{118}$ A. Deisher, ${ }^{118}$ J. Duris, ${ }^{118}$ S. Erhan, ${ }^{118}$ C. Farrell, ${ }^{118}$ J. Hauser, ${ }^{118}$ M. Ignatenko, ${ }^{118}$ C. Jarvis, ${ }^{118}$ C. Plager,${ }^{118}$ G. Rakness, ${ }^{118}$ P. Schlein, ${ }^{118, a}$ J. Tucker, ${ }^{18}$ V. Valuev, ${ }^{118}$ J. Babb, ${ }^{119}$ R. Clare, ${ }^{119}$ J. Ellison, ${ }^{119}$ J. W. Gary, ${ }^{119}$ F. Giordano, ${ }^{119}$ G. Hanson, ${ }^{119}$ G. Y. Jeng, ${ }^{119}$ S. C. Kao, ${ }^{119}$ H. Liu, ${ }^{119}$ O. R. Long, ${ }^{119}$ A. Luthra, ${ }^{119}$ H. Nguyen, ${ }^{119}$ S. Paramesvaran, ${ }^{119}$ J. Sturdy, ${ }^{119}$ S. Sumowidagdo, ${ }^{119}$ R. Wilken, ${ }^{119}$ S. Wimpenny, ${ }^{119}$ W. Andrews, ${ }^{120}$ J. G. Branson, ${ }^{120}$ G. B. Cerati, ${ }^{120}$ D. Evans, ${ }^{120}$ F. Golf,${ }^{120}$ A. Holzner, ${ }^{120}$ R. Kelley, ${ }^{120}$ M. Lebourgeois,${ }^{120}$ J. Letts, ${ }^{120}$ B. Mangano, ${ }^{120}$ S. Padhi, ${ }^{120}$ C. Palmer, ${ }^{120}$ G. Petrucciani, ${ }^{120}$ H. Pi,${ }^{120}$ M. Pieri, ${ }^{120}$ R. Ranieri, ${ }^{120}$ M. Sani, ${ }^{120}$ V. Sharma, ${ }^{120}$ S. Simon, ${ }^{120}$ E. Sudano, ${ }^{120}$ M. Tadel, ${ }^{120}$ Y. Tu, ${ }^{120}$ A. Vartak, ${ }^{120}$ S. Wasserbaech, ${ }^{120, \text { uu }}$ F. Würthwein, ${ }^{120}$ A. Yagil, ${ }^{120}$ J. Yoo, ${ }^{120}$ D. Barge, ${ }^{121}$ R. Bellan, ${ }^{121}$ C. Campagnari, ${ }^{121}$ M. D'Alfonso, ${ }^{121}$ T. Danielson, ${ }^{121}$ K. Flowers, ${ }^{121}$ P. Geffert, ${ }^{121}$ J. Incandela, ${ }^{121}$ C. Justus, ${ }^{121}$ P. Kalavase, ${ }^{121}$ S. A. Koay, ${ }^{121}$ D. Kovalskyi, ${ }^{121, b}$ V. Krutelyov,${ }^{121}$ S. Lowette, ${ }^{121}$ N. Mccoll, ${ }^{121}$ 
S. D. Mullin, ${ }^{121}$ V. Pavlunin, ${ }^{121}$ F. Rebassoo, ${ }^{121}$ J. Ribnik, ${ }^{121}$ J. Richman, ${ }^{121}$ R. Rossin, ${ }^{121}$ D. Stuart, ${ }^{121}$ W. To, ${ }^{121}$ J. R. Vlimant, ${ }^{121}$ C. West, ${ }^{121}$ A. Apresyan, ${ }^{122}$ A. Bornheim, ${ }^{122}$ J. Bunn, ${ }^{122}$ Y. Chen, ${ }^{122}$ J. Duarte, ${ }^{122}$ M. Gataullin, ${ }^{122}$ Y. Ma, ${ }^{122}$ A. Mott, ${ }^{122}$ H. B. Newman, ${ }^{122}$ C. Rogan, ${ }^{122}$ K. Shin, ${ }^{122}$ V. Timciuc, ${ }^{122}$ P. Traczyk, ${ }^{122}$ J. Veverka, ${ }^{122}$ R. Wilkinson, ${ }^{122}$ Y. Yang, ${ }^{122}$ R. Y. Zhu, ${ }^{122}$ B. Akgun, ${ }^{123}$ R. Carroll, ${ }^{123}$ T. Ferguson, ${ }^{123}$ Y. Iiyama, ${ }^{123}$ D. W. Jang, ${ }^{123}$ S. Y. Jun, ${ }^{123}$ Y. F. Liu, ${ }^{123}$ M. Paulini, ${ }^{123}$ J. Russ, ${ }^{123}$ H. Vogel, ${ }^{123}$ I. Vorobiev, ${ }^{123}$ J. P. Cumalat, ${ }^{124}$ M. E. Dinardo, ${ }^{124}$ B. R. Drell, ${ }^{124}$ C. J. Edelmaier, ${ }^{124}$ W. T. Ford, ${ }^{124}$ A. Gaz, ${ }^{124}$ B. Heyburn, ${ }^{124}$ E. Luiggi Lopez, ${ }^{124}$ U. Nauenberg, ${ }^{124}$ J. G. Smith, ${ }^{124}$ K. Stenson, ${ }^{124}$ K. A. Ulmer, ${ }^{124}$ S. R. Wagner, ${ }^{124}$ S. L. Zang, ${ }^{124}$ L. Agostino, ${ }^{125}$ J. Alexander, ${ }^{125}$ A. Chatterjee, ${ }^{125}$ N. Eggert, ${ }^{125}$ L. K. Gibbons, ${ }^{125}$ B. Heltsley, ${ }^{125}$ W. Hopkins, ${ }^{125}$ A. Khukhunaishvili, ${ }^{125}$ B. Kreis, ${ }^{125}$ G. Nicolas Kaufman, ${ }^{125}$ J. R. Patterson, ${ }^{125}$ D. Puigh, ${ }^{125}$ A. Ryd,${ }^{125}$ E. Salvati, ${ }^{125}$ X. Shi,${ }^{125}$ W. Sun, ${ }^{125}$ W. D. Teo, ${ }^{125}$ J. Thom, ${ }^{125}$ J. Thompson, ${ }^{125}$ J. Vaughan, ${ }^{125}$ Y. Weng, ${ }^{125}$ L. Winstrom, ${ }^{125}$ P. Wittich, ${ }^{125}$ A. Biselli, ${ }^{126}$ G. Cirino, ${ }^{126}$

D. Winn, ${ }^{126}$ S. Abdullin, ${ }^{127}$ M. Albrow, ${ }^{127}$ J. Anderson, ${ }^{127}$ G. Apollinari, ${ }^{127}$ M. Atac, ${ }^{127}$ J. A. Bakken, ${ }^{127}$

L. A. T. Bauerdick, ${ }^{127}$ A. Beretvas, ${ }^{127}$ J. Berryhill, ${ }^{127}$ P. C. Bhat,${ }^{127}$ I. Bloch,${ }^{127}$ K. Burkett, ${ }^{127}$ J. N. Butler, ${ }^{127}$ V. Chetluru, ${ }^{127}$ H. W. K. Cheung, ${ }^{127}$ F. Chlebana, ${ }^{127}$ S. Cihangir, ${ }^{127}$ W. Cooper, ${ }^{127}$ D. P. Eartly, ${ }^{127}$ V. D. Elvira, ${ }^{127}$ S. Esen, ${ }^{127}$ I. Fisk, ${ }^{127}$ J. Freeman, ${ }^{127}$ Y. Gao, ${ }^{127}$ E. Gottschalk, ${ }^{127}$ D. Green, ${ }^{127}$ O. Gutsche, ${ }^{127}$ J. Hanlon, ${ }^{127}$ R. M. Harris, ${ }^{127}$ J. Hirschauer, ${ }^{127}$ B. Hooberman, ${ }^{127}$ H. Jensen, ${ }^{127}$ S. Jindariani, ${ }^{127}$ M. Johnson, ${ }^{127}$ U. Joshi, ${ }^{127}$ B. Klima, ${ }^{127}$ K. Kousouris, ${ }^{127}$ S. Kunori, ${ }^{127}$ S. Kwan, ${ }^{127}$ C. Leonidopoulos, ${ }^{127}$ P. Limon, ${ }^{127}$ D. Lincoln, ${ }^{127}$ R. Lipton, ${ }^{127}$ J. Lykken, ${ }^{127}$ K. Maeshima, ${ }^{127}$ J. M. Marraffino, ${ }^{127}$ D. Mason, ${ }^{127}$ P. McBride, ${ }^{127}$ T. Miao, ${ }^{127}$ K. Mishra, ${ }^{127}$ S. Mrenna, ${ }^{127}$ Y. Musienko, ${ }^{127, v v}$ C. Newman-Holmes, ${ }^{127}$ V. O’Dell, ${ }^{127}$ J. Pivarski, ${ }^{127}$ R. Pordes, ${ }^{127}$ O. Prokofyev, ${ }^{127}$ T. Schwarz, ${ }^{127}$ E. Sexton-Kennedy, ${ }^{127}$ S. Sharma, ${ }^{127}$ W. J. Spalding, ${ }^{127}$ L. Spiegel, ${ }^{127}$ P. Tan, ${ }^{127}$ L. Taylor, ${ }^{127}$ S. Tkaczyk, ${ }^{127}$ L. Uplegger,${ }^{127}$ E. W. Vaandering,,${ }^{127}$ R. Vidal, ${ }^{127}$ J. Whitmore, ${ }^{127}$ W. Wu, ${ }^{127}$ F. Yang, ${ }^{127}$ F. Yumiceva, ${ }^{127}$ J. C. Yun, ${ }^{127}$ D. Acosta, ${ }^{128}$ P. Avery, ${ }^{128}$ D. Bourilkov, ${ }^{128}$ M. Chen, ${ }^{128}$ S. Das, ${ }^{128}$ M. De Gruttola, ${ }^{128}$ G. P. Di Giovanni, ${ }^{128}$ D. Dobur, ${ }^{128}$ A. Drozdetskiy, ${ }^{128}$ R. D. Field, ${ }^{128}$ M. Fisher, ${ }^{128}$ Y. Fu, ${ }^{128}$ I. K. Furic ${ }^{128}$ J. Gartner, ${ }^{128}$ S. Goldberg, ${ }^{128}$ J. Hugon, ${ }^{128}$ B. Kim, ${ }^{128}$ J. Konigsberg, ${ }^{128}$ A. Korytov, ${ }^{128}$ A. Kropivnitskaya, ${ }^{128}$ T. Kypreos, ${ }^{128}$ J. F. Low, ${ }^{128}$ K. Matchev, ${ }^{128}$ G. Mitselmakher, ${ }^{128}$ L. Muniz, ${ }^{128}$ P. Myeonghun, ${ }^{128}$ R. Remington, ${ }^{128}$ A. Rinkevicius, ${ }^{128}$ M. Schmitt, ${ }^{128}$ B. Scurlock, ${ }^{128}$ P. Sellers, ${ }^{128}$ N. Skhirtladze, ${ }^{128}$ M. Snowball, ${ }^{128}$ D. Wang, ${ }^{128}$ J. Yelton, ${ }^{128}$ M. Zakaria, ${ }^{128}$ V. Gaultney, ${ }^{129}$ L. M. Lebolo, ${ }^{129}$ S. Linn, ${ }^{129}$ P. Markowitz, ${ }^{129}$ G. Martinez, ${ }^{129}$ J. L. Rodriguez, ${ }^{129}$ T. Adams, ${ }^{130}$ A. Askew, ${ }^{130}$ J. Bochenek, ${ }^{130}$ J. Chen, ${ }^{130}$ B. Diamond, ${ }^{130}$ S. V. Gleyzer, ${ }^{130}$ J. Haas, ${ }^{130}$ S. Hagopian, ${ }^{130}$ V. Hagopian, ${ }^{130}$ M. Jenkins, ${ }^{130}$ K. F. Johnson, ${ }^{130}$ H. Prosper,${ }^{130}$ S. Sekmen, ${ }^{130}$ V. Veeraraghavan, ${ }^{130}$ M. M. Baarmand, ${ }^{131}$ B. Dorney ${ }^{131}$ M. Hohlmann, ${ }^{131}$ H. Kalakhety,${ }^{131}$ I. Vodopiyanov, ${ }^{131}$ M. R. Adams, ${ }^{132}$ I. M. Anghel, ${ }^{132}$ L. Apanasevich, ${ }^{132}$ Y. Bai, ${ }^{132}$ V. E. Bazterra, ${ }^{132}$ R. R. Betts, ${ }^{132}$ J. Callner, ${ }^{132}$ R. Cavanaugh, ${ }^{132}$ C. Dragoiu, ${ }^{132}$ L. Gauthier, ${ }^{132}$ C.E. Gerber, ${ }^{132}$ D. J. Hofman, ${ }^{132}$ S. Khalatyan, ${ }^{132}$ G. J. Kunde, ${ }^{132, w w}$ F. Lacroix, ${ }^{132}$ M. Malek, ${ }^{132}$ C. O'Brien, ${ }^{132}$ C. Silkworth, ${ }^{132}$ C. Silvestre, ${ }^{132}$ A. Smoron, ${ }^{132}$ D. Strom, ${ }^{132}$ N. Varelas, ${ }^{132}$ U. Akgun, ${ }^{133}$ E. A. Albayrak, ${ }^{133}$ B. Bilki, ${ }^{133}$ W. Clarida,${ }^{133}$ F. Duru, ${ }^{133}$ C. K. Lae, ${ }^{133}$ E. McCliment, ${ }^{133}$ J.-P. Merlo, ${ }^{133}$ H. Mermerkaya, ${ }^{133, x x}$ A. Mestvirishvili, ${ }^{133}$ A. Moeller, ${ }^{133}$ J. Nachtman, ${ }^{133}$ C. R. Newsom, ${ }^{133}$ E. Norbeck, ${ }^{133}$ J. Olson, ${ }^{133}$ Y. Onel, ${ }^{133}$ F. Ozok, ${ }^{133}$ S. Sen, ${ }^{133}$ J. Wetzel, ${ }^{133}$ T. Yetkin, ${ }^{133}$ K. Yi, ${ }^{133}$ B. A. Barnett, ${ }^{134}$ B. Blumenfeld, ${ }^{134}$ A. Bonato, ${ }^{134}$ C. Eskew, ${ }^{134}$ D. Fehling, ${ }^{134}$ G. Giurgiu, ${ }^{134}$ A. V. Gritsan, ${ }^{134}$ K. Grizzard, ${ }^{134}$ Z. J. Guo, ${ }^{134}$ G. Hu, ${ }^{134}$ P. Maksimovic, ${ }^{134}$ S. Rappoccio,${ }^{134}$ M. Swartz ${ }^{134}$ N. V. Tran,${ }^{134}$ A. Whitbeck, ${ }^{134}$ P. Baringer, ${ }^{135}$ A. Bean, ${ }^{135}$ G. Benelli, ${ }^{135}$ O. Grachov,${ }^{135}$ R. P. Kenny Iii, ${ }^{135}$ M. Murray, ${ }^{135}$ D. Noonan, ${ }^{135}$ S. Sanders, ${ }^{135}$ R. Stringer, ${ }^{135}$ J. S. Wood, ${ }^{135}$ V. Zhukova, ${ }^{135}$ A. F. Barfuss, ${ }^{136}$ T. Bolton, ${ }^{136}$ I. Chakaberia, ${ }^{136}$ A. Ivanov, ${ }^{136}$ S. Khalil, ${ }^{136}$ M. Makouski ${ }^{136}$ Y. Maravin, ${ }^{136}$ S. Shrestha ${ }^{136}$ I. Svintradze,${ }^{136}$ J. Gronberg, ${ }^{137}$ D. Lange,${ }^{137}$ D. Wright,${ }^{137}$ A. Baden, ${ }^{138}$ M. Boutemeur, ${ }^{138}$ S. C. Eno, ${ }^{138}$ D. Ferencek, ${ }^{138}$ J. A. Gomez, ${ }^{138}$ N. J. Hadley, ${ }^{138}$ R. G. Kellogg, ${ }^{138}$ M. Kirn, ${ }^{138}$ Y. Lu, ${ }^{138}$ A. C. Mignerey, ${ }^{138}$ K. Rossato, ${ }^{138}$ P. Rumerio, ${ }^{138}$ F. Santanastasio, ${ }^{138}$ A. Skuja, ${ }^{138}$ J. Temple, ${ }^{138}$ M. B. Tonjes, ${ }^{138}$ S. C. Tonwar, ${ }^{138}$ E. Twedt, ${ }^{138}$ B. Alver, ${ }^{139}$ G. Bauer, ${ }^{139}$ J. Bendavid, ${ }^{139}$ W. Busza, ${ }^{139}$ E. Butz ${ }^{139}$ I. A. Cali, ${ }^{139}$ M. Chan, ${ }^{139}$ V. Dutta, ${ }^{139}$ P. Everaerts, ${ }^{139}$ G. Gomez Ceballos, ${ }^{139}$ M. Goncharov, ${ }^{139}$ K. A. Hahn, ${ }^{139}$ P. Harris, ${ }^{139}$ Y. Kim, ${ }^{139}$ M. Klute, ${ }^{139}$ Y.-J. Lee, ${ }^{139}$ W. Li ${ }^{139}$ C. Loizides, ${ }^{139}$ P. D. Luckey, ${ }^{139}$ T. Ma ${ }^{139}$ S. Nahn, ${ }^{139}$ C. Paus,${ }^{139}$ D. Ralph,${ }^{139}$ C. Roland,${ }^{139}$ G. Roland, ${ }^{139}$ M. Rudolph ${ }^{139}$ G. S. F. Stephans, ${ }^{139}$ F. Stöckli, ${ }^{139}$ K. Sumorok, ${ }^{139}$ K. Sung, ${ }^{139}$ D. Velicanu, ${ }^{139}$ E. A. Wenger ${ }^{139}$ R. Wolf,${ }^{139}$ B. Wyslouch, ${ }^{139}$ S. Xie, ${ }^{139}$ M. Yang, ${ }^{139}$ Y. Yilmaz, ${ }^{139}$ A. S. Yoon, ${ }^{139}$ M. Zanetti, ${ }^{139}$ S. I. Cooper, ${ }^{140}$ P. Cushman, ${ }^{140}$ B. Dahmes, ${ }^{140}$ A. De Benedetti, ${ }^{140}$ G. Franzoni, ${ }^{140}$ A. Gude, ${ }^{140}$ J. Haupt ${ }^{140}$ K. Klapoetke, ${ }^{140}$ Y. Kubota, ${ }^{140}$ J. Mans,${ }^{140}$ N. Pastika,${ }^{140}$ V. Rekovic, ${ }^{140}$ R. Rusack,${ }^{140}$ M. Sasseville, ${ }^{140}$ A. Singovsky, ${ }^{140}$ N. Tambe, ${ }^{140}$ J. Turkewitz, ${ }^{140}$ L. M. Cremaldi, ${ }^{141}$ R. Godang,${ }^{141}$ R. Kroeger,${ }^{141}$ L. Perera, ${ }^{141}$ 
R. Rahmat, ${ }^{141}$ D. A. Sanders, ${ }^{141}$ D. Summers, ${ }^{141}$ K. Bloom, ${ }^{142}$ S. Bose, ${ }^{142}$ J. Butt, ${ }^{142}$ D. R. Claes, ${ }^{142}$ A. Dominguez, ${ }^{142}$ M. Eads, ${ }^{142}$ P. Jindal, ${ }^{142}$ J. Keller,${ }^{142}$ T. Kelly, ${ }^{142}$ I. Kravchenko, ${ }^{142}$ J. Lazo-Flores, ${ }^{142}$ H. Malbouisson, ${ }^{142}$ S. Malik, ${ }^{142}$ G. R. Snow, ${ }^{142}$ U. Baur, ${ }^{143}$ A. Godshalk, ${ }^{143}$ I. Iashvili, ${ }^{143}$ S. Jain,,${ }^{143}$ A. Kharchilava, ${ }^{143}$ A. Kumar, ${ }^{143}$ K. Smith, ${ }^{143}$ Z. Wan, ${ }^{143}$ G. Alverson, ${ }^{144}$ E. Barberis, ${ }^{144}$ D. Baumgartel,,${ }^{144}$ O. Boeriu, ${ }^{144}$ M. Chasco, ${ }^{144}$ S. Reucroft, ${ }^{144}$ J. Swain, ${ }^{144}$ D. Trocino, ${ }^{144}$ D. Wood, ${ }^{144}$ J. Zhang, ${ }^{144}$ A. Anastassov, ${ }^{145}$ A. Kubik, ${ }^{145}$ N. Mucia, ${ }^{145}$ N. Odell, ${ }^{145}$ R. A. Ofierzynski, ${ }^{145}$ B. Pollack, ${ }^{145}$ A. Pozdnyakov, ${ }^{145}$ M. Schmitt,,${ }^{145}$

S. Stoynev, ${ }^{145}$ M. Velasco, ${ }^{145}$ S. Won, ${ }^{145}$ L. Antonelli, ${ }^{146}$ D. Berry, ${ }^{146}$ A. Brinkerhoff, ${ }^{146}$ M. Hildreth, ${ }^{146}$ C. Jessop, ${ }^{146}$ D. J. Karmgard, ${ }^{146}$ J. Kolb, ${ }^{146}$ T. Kolberg, ${ }^{146}$ K. Lannon, ${ }^{146}$ W. Luo, ${ }^{146}$ S. Lynch, ${ }^{146}$ N. Marinelli, ${ }^{146}$ D. M. Morse, ${ }^{146}$ T. Pearson, ${ }^{146}$ R. Ruchti, ${ }^{146}$ J. Slaunwhite, ${ }^{146}$ N. Valls, ${ }^{146}$ M. Wayne, ${ }^{146}$ J. Ziegler, ${ }^{146}$ B. Bylsma, ${ }^{147}$ L. S. Durkin, ${ }^{147}$ C. Hill,${ }^{147}$ P. Killewald,${ }^{147}$ K. Kotov, ${ }^{147}$ T. Y. Ling,${ }^{147}$ M. Rodenburg ${ }^{147}$ C. Vuosalo, ${ }^{147}$ G. Williams, ${ }^{147}$ N. Adam, ${ }^{148}$ E. Berry, ${ }^{148}$ P. Elmer, ${ }^{148}$ D. Gerbaudo, ${ }^{148}$ V. Halyo, ${ }^{148}$ P. Hebda, ${ }^{148}$ A. Hunt, ${ }^{148}$ E. Laird ${ }^{148}$ D. Lopes Pegna, ${ }^{148}$ D. Marlow, ${ }^{148}$ T. Medvedeva, ${ }^{148}$ M. Mooney, ${ }^{148}$ J. Olsen, ${ }^{148}$ P. Piroué, ${ }^{148}$ X. Quan, ${ }^{148}$ H. Saka, ${ }^{148}$ D. Stickland, ${ }^{148}$ C. Tully, ${ }^{148}$ J. S. Werner, ${ }^{148}$ A. Zuranski, ${ }^{148}$ J. G. Acosta, ${ }^{149}$ X. T. Huang, ${ }^{149}$ A. Lopez, ${ }^{149}$ H. Mendez, ${ }^{149}$ S. Oliveros, ${ }^{149}$ J.E. Ramirez Vargas, ${ }^{149}$ A. Zatserklyaniy,${ }^{149}$ E. Alagoz, ${ }^{150}$ V. E. Barnes, ${ }^{150}$ G. Bolla, ${ }^{150}$ L. Borrello, ${ }^{150}$ D. Bortoletto, ${ }^{150}$ M. De Mattia, ${ }^{150}$ A. Everett,${ }^{150}$ L. Gutay, ${ }^{150}$ Z. Hu, ${ }^{150}$ M. Jones, ${ }^{150}$ O. Koybasi, ${ }^{150}$ M. Kress, ${ }^{150}$ A. T. Laasanen, ${ }^{150}$ N. Leonardo, ${ }^{150}$ V. Maroussov, ${ }^{150}$ P. Merkel, ${ }^{150}$ D. H. Miller,${ }^{150}$ N. Neumeister, ${ }^{150}$ I. Shipsey, ${ }^{150}$ D. Silvers, ${ }^{150}$ A. Svyatkovskiy, ${ }^{150}$ M. Vidal Marono, ${ }^{150}$ H. D. Yoo, ${ }^{150}$ J. Zablocki, ${ }^{150}$ Y. Zheng, ${ }^{150}$ S. Guragain, ${ }^{151}$ N. Parashar, ${ }^{151}$ A. Adair, ${ }^{152}$ C. Boulahouache,${ }^{152}$ K. M. Ecklund, ${ }^{152}$ F. J. M. Geurts, ${ }^{152}$ B. P. Padley, ${ }^{152}$ R. Redjimi, ${ }^{152}$ J. Roberts, ${ }^{152}$ J. Zabel, ${ }^{152}$ B. Betchart, ${ }^{153}$ A. Bodek,${ }^{153}$ Y. S. Chung, ${ }^{153}$ R. Covarelli, ${ }^{153}$ P. de Barbaro, ${ }^{153}$ R. Demina, ${ }^{153}$ Y. Eshaq, ${ }^{153}$ H. Flacher, ${ }^{153}$ A. Garcia-Bellido, ${ }^{153}$ P. Goldenzweig, ${ }^{153}$ Y. Gotra, ${ }^{153}$

J. Han,${ }^{153}$ A. Harel,${ }^{153}$ D. C. Miner, ${ }^{153}$ G. Petrillo, ${ }^{153}$ W. Sakumoto, ${ }^{153}$ D. Vishnevskiy, ${ }^{153}$ M. Zielinski, ${ }^{153}$

A. Bhatti, ${ }^{154}$ R. Ciesielski, ${ }^{154}$ L. Demortier, ${ }^{154}$ K. Goulianos,${ }^{154}$ G. Lungu, ${ }^{154}$ S. Malik, ${ }^{154}$ C. Mesropian, ${ }^{154}$ S. Arora, ${ }^{155}$ O. Atramentov, ${ }^{155}$ A. Barker, ${ }^{155}$ C. Contreras-Campana, ${ }^{155}$ E. Contreras-Campana, ${ }^{155}$ D. Duggan, ${ }^{155}$ Y. Gershtein, ${ }^{155}$ R. Gray, ${ }^{155}$ E. Halkiadakis, ${ }^{155}$ D. Hidas, ${ }^{155}$ D. Hits, ${ }^{155}$ A. Lath, ${ }^{155}$ S. Panwalkar, ${ }^{155}$ M. Park, ${ }^{155}$ R. Patel, ${ }^{155}$ A. Richards, ${ }^{155}$ K. Rose,${ }^{155}$ S. Schnetzer ${ }^{155}$ S. Somalwar, ${ }^{155}$ R. Stone,${ }^{155}$ S. Thomas, ${ }^{155}$ G. Cerizza,${ }^{156}$ M. Hollingsworth, ${ }^{156}$ S. Spanier, ${ }^{156}$ Z. C. Yang, ${ }^{156}$ A. York, ${ }^{156}$ R. Eusebi, ${ }^{157}$ W. Flanagan, ${ }^{157}$ J. Gilmore, ${ }^{157}$ A. Gurrola, ${ }^{157}$ T. Kamon, ${ }^{157, y y}$ V. Khotilovich, ${ }^{157}$ R. Montalvo, ${ }^{157}$ I. Osipenkov, ${ }^{157}$ Y. Pakhotin, ${ }^{157}$ A. Perloff, ${ }^{157}$ J. Roe, ${ }^{157}$ A. Safonov, ${ }^{157}$ S. Sengupta, ${ }^{157}$ I. Suarez ${ }^{157}$ A. Tatarinov, ${ }^{157}$ D. Toback, ${ }^{157}$ N. Akchurin, ${ }^{158}$ C. Bardak,${ }^{158}$ J. Damgov, ${ }^{158}$ P. R. Dudero, ${ }^{158}$ C. Jeong, ${ }^{158}$ K. Kovitanggoon, ${ }^{158}$ S. W. Lee, ${ }^{158}$ T. Libeiro, ${ }^{158}$ P. Mane, ${ }^{158}$ Y. Roh,${ }^{158}$ A. Sill, ${ }^{158}$ I. Volobouev, ${ }^{158}$ R. Wigmans, ${ }^{158}$ E. Yazgan, ${ }^{158}$ E. Appelt, ${ }^{159}$ E. Brownson, ${ }^{159}$ D. Engh, ${ }^{159}$ C. Florez, ${ }^{159}$ W. Gabella, ${ }^{159}$ M. Issah, ${ }^{159}$ W. Johns, ${ }^{159}$ C. Johnston, ${ }^{159}$ P. Kurt, ${ }^{159}$ C. Maguire, ${ }^{159}$ A. Melo, ${ }^{159}$ P. Sheldon, ${ }^{159}$ B. Snook, ${ }^{159}$ S. Tuo, ${ }^{159}$ J. Velkovska, ${ }^{159}$ M. W. Arenton, ${ }^{160}$ M. Balazs, ${ }^{160}$ S. Boutle, ${ }^{160}$ B. Cox,${ }^{160}$ B. Francis, ${ }^{160}$ S. Goadhouse, ${ }^{160}$ J. Goodell, ${ }^{160}$ R. Hirosky, ${ }^{160}$ A. Ledovskoy,${ }^{160}$ C. Lin,${ }^{160}$ C. Neu, ${ }^{160}$ J. Wood, ${ }^{160}$ R. Yohay, ${ }^{160}$ S. Gollapinni, ${ }^{161}$ R. Harr, ${ }^{161}$ P. E. Karchin, ${ }^{161}$ C. Kottachchi Kankanamge Don, ${ }^{161}$ P. Lamichhane, ${ }^{161}$ M. Mattson, ${ }^{161}$ C. Milstène, ${ }^{161}$ A. Sakharov,${ }^{161}$ M. Anderson, ${ }^{162}$ M. Bachtis, ${ }^{162}$ D. Belknap, ${ }^{162}$ J. N. Bellinger, ${ }^{162}$ D. Carlsmith ${ }^{162}$ M. Cepeda, ${ }^{162}$ S. Dasu, ${ }^{162}$ J. Efron, ${ }^{162}$ E. Friis, ${ }^{162}$ L. Gray, ${ }^{162}$ K. S. Grogg, ${ }^{162}$ M. Grothe, ${ }^{162}$ R. Hall-Wilton, ${ }^{162}$ M. Herndon, ${ }^{162}$ A. Hervé, ${ }^{162}$ P. Klabbers, ${ }^{162}$ J. Klukas, ${ }^{162}$ A. Lanaro, ${ }^{162}$ C. Lazaridis, ${ }^{162}$ J. Leonard, ${ }^{162}$ R. Loveless, ${ }^{162}$ A. Mohapatra, ${ }^{162}$ I. Ojalvo, ${ }^{162}$ W. Parker, ${ }^{162}$ I. Ross, ${ }^{162}$ A. Savin, ${ }^{162}$ W. H. Smith, ${ }^{162}$ J. Swanson, ${ }^{162}$ and M. Weinberg ${ }^{162}$

\title{
(CMS Collaboration)
}

\author{
${ }^{1}$ Yerevan Physics Institute, Yerevan, Armenia \\ ${ }^{2}$ Institut für Hochenergiephysik der OeAW, Wien, Austria \\ ${ }^{3}$ National Centre for Particle and High Energy Physics, Minsk, Belarus \\ ${ }^{4}$ Universiteit Antwerpen, Antwerpen, Belgium \\ ${ }^{5}$ Vrije Universiteit Brussel, Brussel, Belgium \\ ${ }^{6}$ Université Libre de Bruxelles, Bruxelles, Belgium \\ ${ }^{7}$ Ghent University, Ghent, Belgium \\ ${ }^{8}$ Université Catholique de Louvain, Louvain-la-Neuve, Belgium \\ ${ }^{9}$ Université de Mons, Mons, Belgium \\ ${ }^{10}$ Centro Brasileiro de Pesquisas Fisicas, Rio de Janeiro, Brazil \\ ${ }^{11}$ Universidade do Estado do Rio de Janeiro, Rio de Janeiro, Brazil
}


${ }^{12}$ Instituto de Fisica Teorica, Universidade Estadual Paulista, Sao Paulo, Brazil

${ }^{13}$ Institute for Nuclear Research and Nuclear Energy, Sofia, Bulgaria

${ }^{14}$ University of Sofia, Sofia, Bulgaria

${ }^{15}$ Institute of High Energy Physics, Beijing, China

${ }^{16}$ State Key Lab. of Nucl. Phys. and Tech., Peking University, Beijing, China

${ }^{17}$ Universidad de Los Andes, Bogota, Colombia

${ }^{18}$ Technical University of Split, Split, Croatia

${ }^{19}$ University of Split, Split, Croatia

${ }^{20}$ Institute Rudjer Boskovic, Zagreb, Croatia

${ }^{21}$ University of Cyprus, Nicosia, Cyprus

${ }^{22}$ Charles University, Prague, Czech Republic

${ }^{23}$ Academy of Scientific Research and Technology of the Arab Republic of Egypt,

Egyptian Network of High Energy Physics, Cairo, Egypt

${ }^{24}$ National Institute of Chemical Physics and Biophysics, Tallinn, Estonia

${ }^{25}$ Department of Physics, University of Helsinki, Helsinki, Finland

${ }^{26}$ Helsinki Institute of Physics, Helsinki, Finland

${ }^{27}$ Lappeenranta University of Technology, Lappeenranta, Finland

${ }^{28}$ Laboratoire d'Annecy-le-Vieux de Physique des Particules, IN2P3-CNRS, Annecy-le-Vieux, France

${ }^{29}$ DSM/IRFU, CEA/Saclay, Gif-sur-Yvette, France

${ }^{30}$ Laboratoire Leprince-Ringuet, Ecole Polytechnique, IN2P3-CNRS, Palaiseau, France

${ }^{31}$ Institut Pluridisciplinaire Hubert Curien, Université de Strasbourg,

Université de Haute, Alsace Mulhouse CNRS/IN2P3, Strasbourg, France

${ }^{32}$ Centre de Calcul de l'Institut National de Physique Nucleaire et de Physique des Particules (IN2P3), Villeurbanne, France

${ }^{33}$ Université de Lyon, Université Claude Bernard Lyon 1, CNRS-IN2P3, Institut de Physique Nucléaire de Lyon, Villeurbanne, France

${ }^{34}$ Institute of High Energy Physics and Informatization, Tbilisi State University, Tbilisi, Georgia

${ }^{35}$ RWTH Aachen University, I. Physikalisches Institut, Aachen, Germany

${ }^{36}$ RWTH Aachen University, III. Physikalisches Institut A, Aachen, Germany

${ }^{37}$ RWTH Aachen University, III. Physikalisches Institut B, Aachen, Germany

${ }^{38}$ Deutsches Elektronen-Synchrotron, Hamburg, Germany

${ }^{39}$ University of Hamburg, Hamburg, Germany

${ }^{40}$ Institut für Experimentelle Kernphysik, Karlsruhe, Germany

${ }^{41}$ Institute of Nuclear Physics "Demokritos", Aghia Paraskevi, Greece

${ }^{42}$ University of Athens, Athens, Greece

${ }^{43}$ University of Ioánnina, Ioánnina, Greece

${ }^{44}$ KFKI Research Institute for Particle and Nuclear Physics, Budapest, Hungary

${ }^{45}$ Institute of Nuclear Research ATOMKI, Debrecen, Hungary

${ }^{46}$ University of Debrecen, Debrecen, Hungary

${ }^{47}$ Panjab University, Chandigarh, India

${ }^{48}$ University of Delhi, Delhi, India

${ }^{49}$ Saha Institute of Nuclear Physics, Kolkata, India

${ }^{50}$ Bhabha Atomic Research Centre, Mumbai, India

${ }^{51}$ Tata Institute of Fundamental Research-EHEP, Mumbai, India

${ }^{52}$ Tata Institute of Fundamental Research-HECR, Mumbai, India

${ }^{53}$ Institute for Research and Fundamental Sciences (IPM), Tehran, Iran

${ }^{54 a}$ INFN Sezione di Bari, Bari, Italy

${ }^{54 \mathrm{~b}}$ Università di Bari, Bari, Italy

${ }^{54 \mathrm{c}}$ Politecnico di Bari, Bari, Italy

${ }^{55}$ INFN Sezione di Bologna, Bologna, Italy

${ }^{55 \mathrm{~b}}$ Università di Bologna, Bologna, Italy

${ }^{56 a}$ INFN Sezione di Catania, Catania, Italy

${ }^{56 b}$ Università di Catania, Catania, Italy

${ }^{57}$ INFN Sezione di Firenze, Firenze, Italy

${ }^{57 \mathrm{~b}}$ Università di Firenze, Firenze, Italy

${ }^{58}$ INFN Laboratori Nazionali di Frascati, Frascati, Italy

${ }^{59}$ INFN Sezione di Genova, Genova, Italy

${ }^{60 \mathrm{a}}$ INFN Sezione di Milano-Bicocca, Milano, Italy

${ }^{60 \mathrm{~b}}$ Università di Milano-Bicocca, Milano, Italy

${ }^{61 \mathrm{a}}$ INFN Sezione di Napoli, Napoli, Italy

${ }^{61 \mathrm{~b}}$ Università di Napoli "Federico II", Napoli, Italy

${ }^{62 a}$ INFN Sezione di Padova, Padova, Italy

${ }^{62 \mathrm{~b}}$ Università di Padova, Padova, Italy 


\author{
${ }^{62 \mathrm{c}}$ Università di Trento (Trento), Padova, Italy \\ ${ }^{63 a}$ INFN Sezione di Pavia, Pavia, Italy \\ ${ }^{63 \mathrm{~b}}$ Università di Pavia, Pavia, Italy \\ ${ }^{64 a}$ INFN Sezione di Perugia, Perugia, Italy \\ ${ }^{64 \mathrm{~b}}$ Università di Perugia, Perugia, Italy \\ ${ }^{65}$ INFN Sezione di Pisa, Pisa, Italy \\ ${ }^{65 \mathrm{~b}}$ Università di Pisa, Pisa, Italy \\ ${ }^{65 \mathrm{c} S c u o l a}$ Normale Superiore di Pisa, Pisa, Italy \\ ${ }^{66}$ INFN Sezione di Roma, Roma, Italy \\ ${ }^{66 \mathrm{~b}}$ Università di Roma "La Sapienza”, Roma, Italy \\ ${ }^{67 a}$ INFN Sezione di Torino, Torino, Italy \\ ${ }^{67 \mathrm{~b}}$ Università di Torino, Torino, Italy \\ ${ }^{67 \mathrm{c}}$ Università del Piemonte Orientale (Novara), Torino, Italy \\ ${ }^{68 \mathrm{a}}$ INFN Sezione di Trieste, Trieste, Italy \\ ${ }^{68 \mathrm{~b}}$ Università di Trieste, Trieste, Italy \\ ${ }^{69}$ Kangwon National University, Chunchon, Korea \\ ${ }^{70}$ Kyungpook National University, Daegu, Korea \\ ${ }^{71}$ Chonnam National University, Institute for Universe and Elementary Particles, Kwangju, Korea \\ ${ }^{72}$ Konkuk University, Seoul, Korea \\ ${ }^{73}$ Korea University, Seoul, Korea \\ ${ }^{74}$ University of Seoul, Seoul, Korea \\ ${ }^{75}$ Sungkyunkwan University, Suwon, Korea \\ ${ }^{76}$ Vilnius University, Vilnius, Lithuania \\ ${ }^{77}$ Centro de Investigacion y de Estudios Avanzados del IPN, Mexico City, Mexico \\ ${ }^{78}$ Universidad Iberoamericana, Mexico City, Mexico \\ ${ }^{79}$ Benemerita Universidad Autonoma de Puebla, Puebla, Mexico \\ ${ }^{80}$ Universidad Autónoma de San Luis Potosí, San Luis Potosí, Mexico \\ ${ }^{81}$ University of Auckland, Auckland, New Zealand \\ ${ }^{82}$ University of Canterbury, Christchurch, New Zealand \\ ${ }^{83}$ National Centre for Physics, Quaid-I-Azam University, Islamabad, Pakistan \\ ${ }^{84}$ Institute of Experimental Physics, Faculty of Physics, University of Warsaw, Warsaw, Poland \\ ${ }^{85}$ Soltan Institute for Nuclear Studies, Warsaw, Poland \\ ${ }^{86}$ Laboratório de Instrumentação e Física Experimental de Partículas, Lisboa, Portugal \\ ${ }^{87}$ Joint Institute for Nuclear Research, Dubna, Russia \\ ${ }^{88}$ Petersburg Nuclear Physics Institute, Gatchina (St Petersburg), Russia \\ ${ }^{89}$ Institute for Nuclear Research, Moscow, Russia \\ ${ }^{90}$ Institute for Theoretical and Experimental Physics, Moscow, Russia \\ ${ }^{91}$ Moscow State University, Moscow, Russia \\ ${ }^{92}$ P.N. Lebedev Physical Institute, Moscow, Russia \\ ${ }^{93}$ State Research Center of Russian Federation, Institute for High Energy Physics, Protvino, Russia \\ ${ }^{94}$ University of Belgrade, Faculty of Physics and Vinca Institute of Nuclear Sciences, Belgrade, Serbia \\ ${ }^{95}$ Centro de Investigaciones Energéticas Medioambientales y Tecnológicas (CIEMAT), Madrid, Spain \\ ${ }^{96}$ Universidad Autónoma de Madrid, Madrid, Spain \\ ${ }^{97}$ Universidad de Oviedo, Oviedo, Spain \\ ${ }^{98}$ Instituto de Física de Cantabria (IFCA), , USACSIC-Universidad de Cantabria, Santander, Spain \\ ${ }^{99}$ CERN, European Organization for Nuclear Research, Geneva, Switzerland \\ ${ }^{100}$ Paul Scherrer Institut, Villigen, Switzerland \\ ${ }^{101}$ Institute for Particle Physics, ETH Zurich, Zurich, Switzerland \\ ${ }^{102}$ Universität Zürich, Zurich, Switzerland \\ ${ }^{103}$ National Central University, Chung-Li, Taiwan \\ ${ }^{104}$ National Taiwan University (NTU), Taipei, Taiwan \\ ${ }^{105}$ Cukurova University, Adana, Turkey \\ ${ }^{106}$ Middle East Technical University, Physics Department, Ankara, Turkey \\ ${ }^{107}$ Bogazici University, Istanbul, Turkey \\ ${ }^{108}$ National Scientific Center, Kharkov Institute of Physics and Technology, Kharkov, Ukraine \\ ${ }^{109}$ University of Bristol, Bristol, United Kingdom \\ ${ }^{110}$ Rutherford Appleton Laboratory, Didcot, United Kingdom \\ ${ }^{111}$ Imperial College, London, United Kingdom \\ ${ }^{112}$ Brunel University, Uxbridge, United Kingdom \\ ${ }^{113}$ Baylor University, Waco, USA \\ ${ }^{114}$ The University of Alabama, Tuscaloosa, USA
}




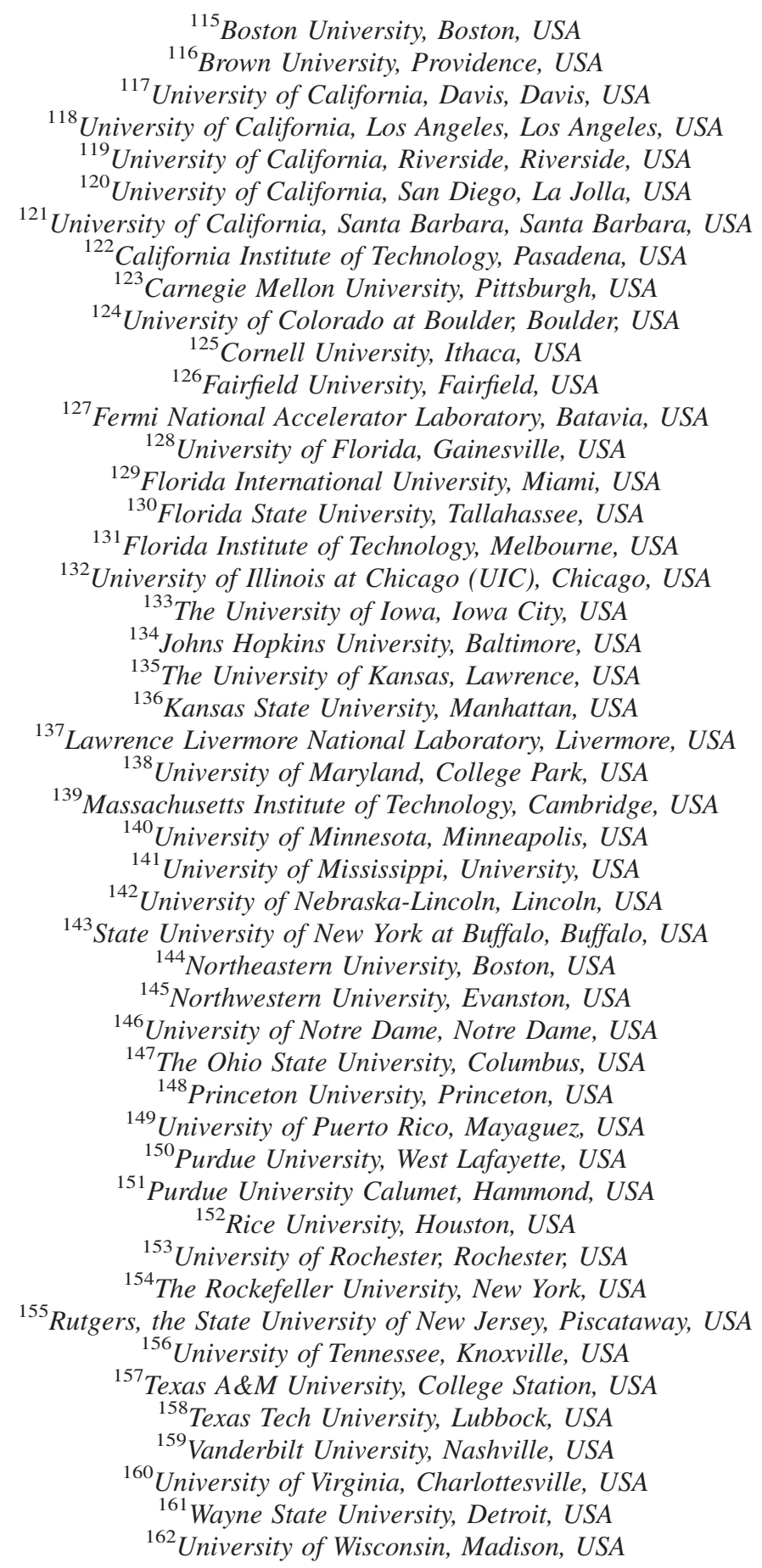

${ }^{\mathrm{a}}$ Deceased.

${ }^{\mathrm{b}}$ Also at CERN, European Organization for Nuclear Research, Geneva, Switzerland

${ }^{\mathrm{c}}$ Also at Universidade Federal do ABC, Santo Andre, Brazil

${ }^{\mathrm{d}}$ Also at California Institute of Technology, Pasadena, USA

${ }^{\mathrm{e}}$ Also at Laboratoire Leprince-Ringuet, Ecole Polytechnique, IN2P3-CNRS, Palaiseau, France

${ }^{\mathrm{f}}$ Also at Suez Canal University, Suez, Egypt

${ }^{\mathrm{g}}$ Also at Cairo University, Cairo, Egypt

${ }^{\mathrm{h}}$ Also at British University, Cairo, Egypt

${ }^{\mathrm{i}}$ Also at Fayoum University, El-Fayoum, Egypt

${ }^{j}$ Also at Ain Shams University, Cairo, Egypt 
${ }^{\mathrm{k}}$ Also at Soltan Institute for Nuclear Studies, Warsaw, Poland

${ }^{1}$ Also at Université de Haute-Alsace, Mulhouse, France

${ }^{\mathrm{m}}$ Also at Moscow State University, Moscow, Russia

${ }^{\mathrm{n}}$ Also at Brandenburg University of Technology, Cottbus, Germany

${ }^{\circ}$ Also at Institute of Nuclear Research ATOMKI, Debrecen, Hungary

${ }^{\mathrm{p}}$ Also at Eötvös Loránd University, Budapest, Hungary

${ }^{\mathrm{q}} \mathrm{Also}$ at Tata Institute of Fundamental Research-HECR, Mumbai, India

${ }^{\mathrm{r}}$ Also at University of Visva-Bharati, Santiniketan, India

${ }^{s}$ Also at Sharif University of Technology, Tehran, Iran

${ }^{\mathrm{t}}$ Also at Isfahan University of Technology, Isfahan, Iran

uAlso at Shiraz University, Shiraz, Iran

${ }^{v}$ Also at Facoltà Ingegneria Università di Roma, Roma, Italy

${ }^{\mathrm{w}}$ Also at Università della Basilicata, Potenza, Italy

${ }^{x}$ Also at Laboratori Nazionali di Legnaro dell' INFN, Legnaro, Italy

${ }^{\mathrm{y}}$ Also at Università degli studi di Siena, Siena, Italy

${ }^{\mathrm{z}}$ Also at Faculty of Physics of University of Belgrade, Belgrade, Serbia

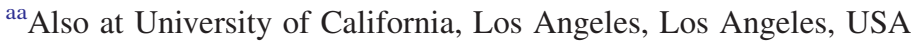

${ }^{\mathrm{bb}}$ Also at University of Florida, Gainesville, USA

${ }^{\mathrm{cc}}$ Also at Université de Genève, Geneva, Switzerland

${ }^{\mathrm{dd}}$ Also at Scuola Normale e Sezione dell' INFN, Pisa, Italy

${ }^{e e}$ Also at INFN Sezione di Roma, Università di Roma "La Sapienza", Roma, Italy, USA

${ }^{\mathrm{ff}}$ Also at University of Athens, Athens, Greece

${ }^{\mathrm{gg}}$ Also at The University of Kansas, Lawrence, USA

${ }^{\text {hh }}$ Also at Paul Scherrer Institut, Villigen, Switzerland

${ }^{i i}$ Also at University of Belgrade, Faculty of Physics and Vinca Institute of Nuclear Sciences, Belgrade, Serbia

${ }^{\mathrm{jj}}$ Also at Institute for Theoretical and Experimental Physics, Moscow, Russia

${ }^{\mathrm{kk}}$ Also at Gaziosmanpasa University, Tokat, Turkey

${ }^{11}$ Also at Adiyaman University, Adiyaman, Turkey

${ }^{\mathrm{mm}}$ Also at The University of Iowa, Iowa City, USA

${ }^{\mathrm{nn}}$ Also at Mersin University, Mersin, Turkey

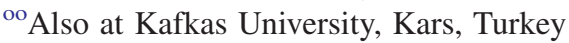

${ }^{\mathrm{pp}}$ Also at Suleyman Demirel University, Isparta, Turkey

${ }^{\mathrm{qq}}$ Also at Ege University, Izmir, Turkey

${ }^{\mathrm{rr}}$ Also at Rutherford Appleton Laboratory, Didcot, United Kingdom

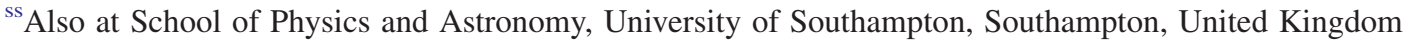

${ }^{\text {tt} A l s o ~ a t ~ I N F N ~ S e z i o n e ~ d i ~ P e r u g i a, ~ U n i v e r s i t a ̀ ~ d i ~ P e r u g i a, ~ P e r u g i a, ~ I t a l y ~}$

${ }^{\mathrm{uu}}$ Also at Utah Valley University, Orem, USA

${ }^{\mathrm{v}}$ Also at Institute for Nuclear Research, Moscow, Russia

${ }^{w w}$ Also at Los Alamos National Laboratory, Los Alamos, USA

${ }^{\mathrm{xx}}$ Also at Erzincan University, Erzincan, Turkey

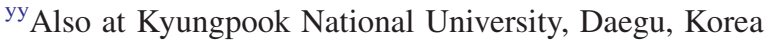

